\title{
Catalytic Asymmetric Synthesis of Thiols
}

Mattia Riccardo Monaco, Sébastien Prévost and Benjamin List

Max-Planck-Institut für Kohlenforschung, Kaiser-Wilhelm-Platz 1, D-45470 Mülheim an der Ruhr, Germany

list@mpi-muelheim.mpg.de

\section{Supplementary Information}

General information....................................................... 2

NMR-experiments for the characterization of heterodimer I................. 3

Further optimization screenings for the thiocarboxylysis step.............. 5

Insights into the acyl-transfer step................................. 6

Synthetic procedures and spectral data ...................................... 7

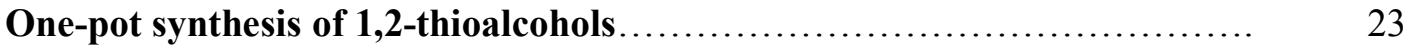

2mmol-scale preparation of compound 5a..................................................... 25

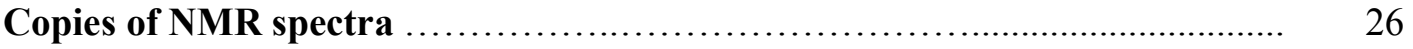

HPLC and GC traces.................................................. 57 


\section{General Information}

Unless otherwise stated, all reagents were purchased from commercial suppliers and used without further purification. All solvents used in the reactions were distilled from appropriate drying agents prior to use. Reactions were monitored by thin layer chromatography on silica gel pre-coated plastic sheets $(0.2 \mathrm{~mm}$, Macherey-Nagel). Visualization was accomplished by irradiation with UV light at $254 \mathrm{~nm}$ and/or $p$-anisaldehyde stain $(0.7 \mathrm{~mL}$ p-anisaldehyde, 250 $\mathrm{mL}$ EtOH, $9.5 \mathrm{~mL}$ conc. $\mathrm{H}_{2} \mathrm{SO}_{4}, 2.7 \mathrm{~mL}$ glacial $\left.\mathrm{AcOH}\right)$ or potassium permanganate stain (1.5 $\mathrm{g} \mathrm{KMnO}_{4}, 10 \mathrm{~g} \mathrm{~K}_{2} \mathrm{CO}_{3}, 1.25 \mathrm{~mL} 10 \% \mathrm{NaOH}, 200 \mathrm{~mL} \mathrm{H} \mathrm{H}_{2} \mathrm{O}$ ). Column chromatography was performed on Merck silica gel (60, particle size 0.040-0.063 mm). Proton and carbon NMR spectra were recorded on Bruker AV-300 or Bruker AV-500 spectrometer in deuterated solvent. Proton chemical shifts are reported in ppm $(\delta)$ relative to tetramethylsilane (TMS) with the solvent resonance employed as the internal standard $\left(\mathrm{CDCl}_{3} \delta 7.26, \mathrm{CD}_{2} \mathrm{Cl}_{2} \delta 5.32\right)$. Data are reported as follows: chemical shift, multiplicity $(\mathrm{s}=$ singlet, $\mathrm{d}=$ doublet, $\mathrm{q}=$ quartet, $\mathrm{Q}=$ quintuplet, $\mathrm{h}=$ heptuplet, $\mathrm{m}=$ multiplet, $\mathrm{br}=$ broad, $\mathrm{cm}=$ centered multiplet), coupling constants $(\mathrm{Hz})$ and integration. ${ }^{13} \mathrm{C}$ chemical shifts are reported in ppm from tetramethylsilane (TMS) with the solvent resonance as the internal standard $\left(\mathrm{CDCl}_{3} \delta 77.23, \mathrm{CD}_{2} \mathrm{Cl}_{2} \delta 53.8\right)$. High resolution mass spectra were determined on a Bruker APEX III FTMS (7 T magnet). Optical rotations were determined with Autopol IV polarimeter (Rudolph Research Analytical) at $589 \mathrm{~nm}$ and $25{ }^{\circ} \mathrm{C}$. Data are reported as follows: $[\alpha]_{\lambda}^{\text {temp }}$, concentration (c in $\mathrm{g} / 100 \mathrm{~mL}$ ), and solvent. The enantiomeric excesses were determined by HPLC or GC analysis employing a chiral stationary phase column specified in the individual experiment, by comparing the samples with the appropriate racemic mixtures. 


\section{NMR-experiments for the characterization of heterodimer I}

This experiment was designed to investigate the heterodimerization between chiral phosphoric acid catalysts and thiocarboxylic acids.

The effect of the addition of thiobenzoic acid ( $8.3 \mathrm{mg}, 30$ equiv.) to a solution of TRIP in deuterated dichloromethane $(1.5 \mathrm{mg}, 0.005 \mathrm{M})$ was analyzed by ${ }^{1} \mathrm{H}-\mathrm{NMR}$ and ${ }^{31} \mathrm{P}-\mathrm{NMR}$ spectroscopy.

The significant shift downfield of the phosphorous signal and the observed changes in the ${ }^{1} \mathrm{H}$ NMR are in agreement with the formation of a novel species: heterodimer I. These data are in accordance with the previously reported self-assembly of TRIP with carboxylic acids. ${ }^{1}$

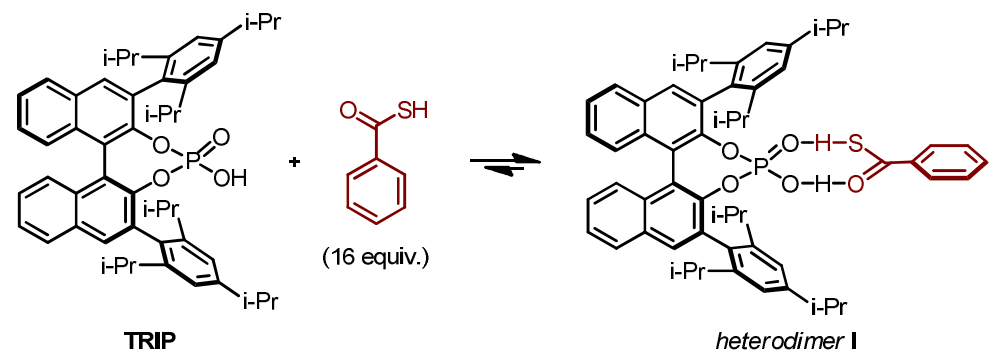

\section{${ }^{31} \mathbf{P}$ - NMR}

TRIP: 2.36 ppm
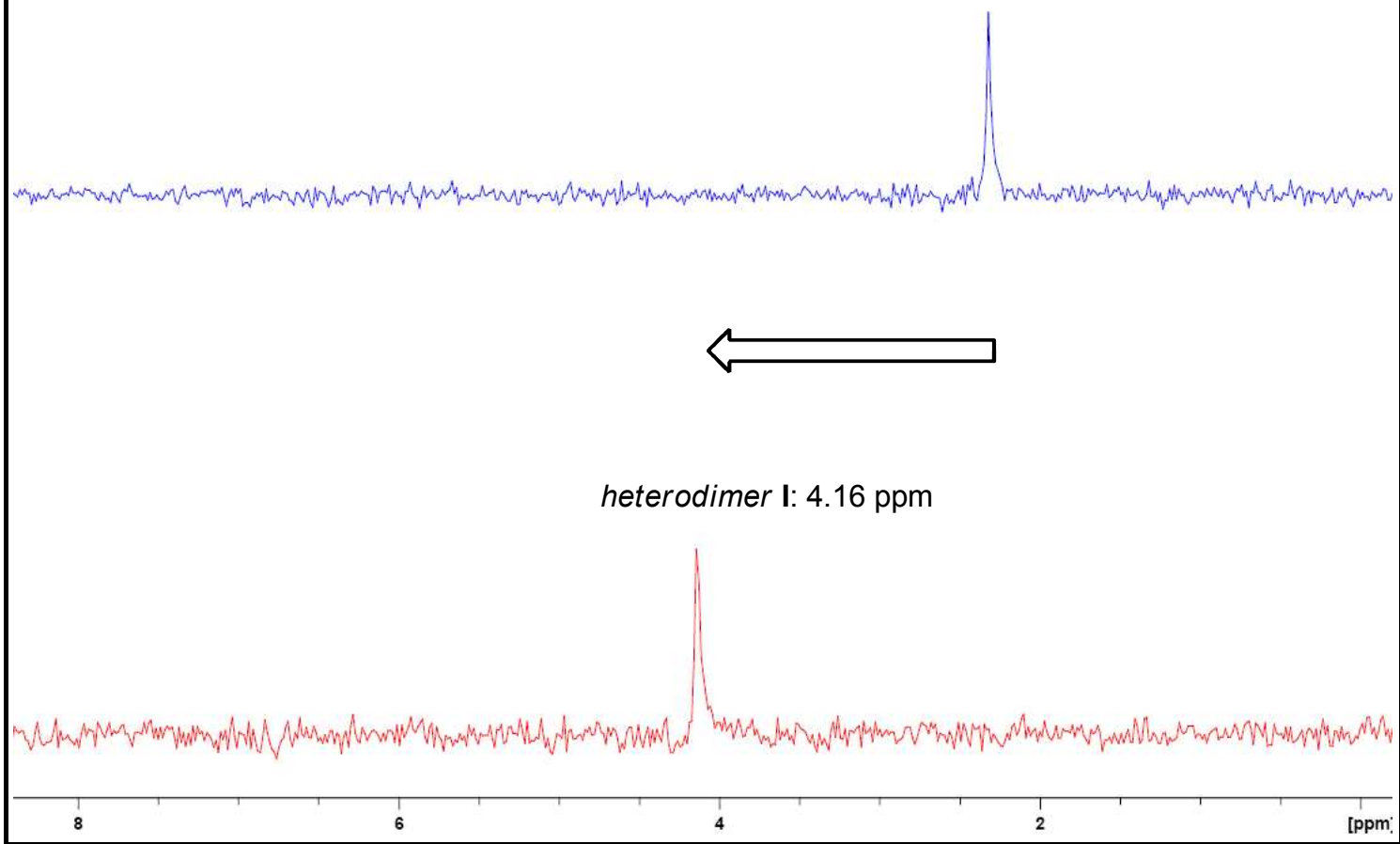

\section{${ }^{1} \mathbf{H}-\mathbf{N M R}$}

\footnotetext{
${ }^{1}$ Monaco, M. R.; Poladura, B.; Diaz de los Bernardos, M.; Leutzsch, M.; Goddard, R.; List, B. Angew. Chem. Int. Ed. 2014, 53, 7063.
} 


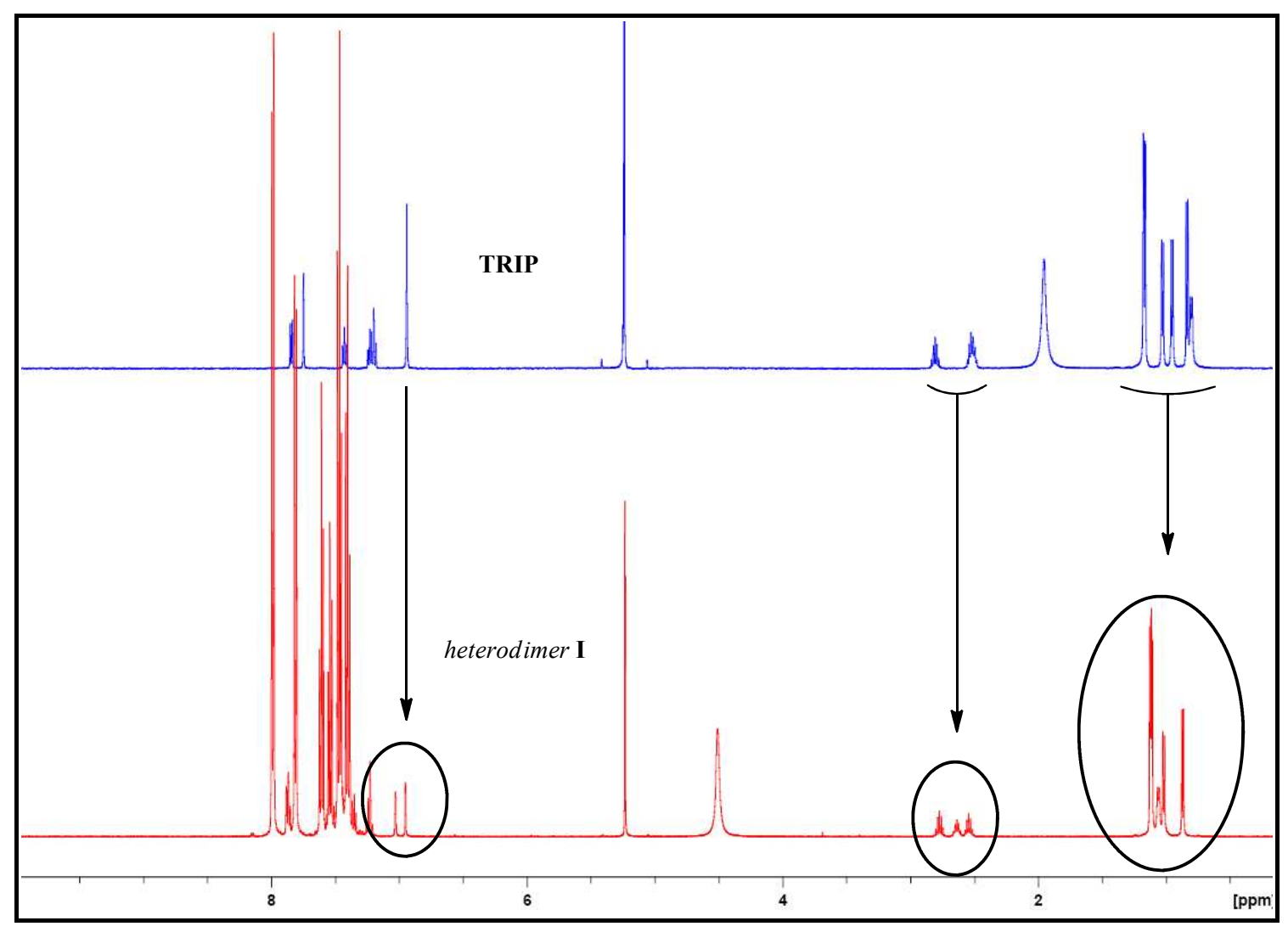




\section{Further optimization screenings for the thiocarboxylysis step}

Table S1. Screening of temperature, catalyst (4b and $4 c)$, and concentration in the ring opening of 1a with thiobenzoic acid.

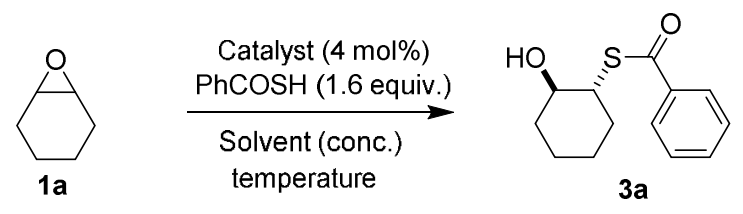

\begin{tabular}{ccccccc}
\hline entry & Solvent & Catalyst & conc. & temperature & Conv ${ }^{[a]}$ & $\mathrm{er}^{[\mathrm{b}]}$ \\
\hline 1 & $\mathrm{CH}_{2} \mathrm{Cl}_{2}$ & $(\mathrm{~S})-\mathbf{4 b}$ & $0.125 \mathrm{M}$ & $-40^{\circ} \mathrm{C}$ & $>95 \%$ & $8: 92$ \\
2 & toluene & $(\mathrm{S})-\mathbf{4 b}$ & $0.125 \mathrm{M}$ & $-40^{\circ} \mathrm{C}$ & $>95 \%$ & $5: 95$ \\
3 & toluene & $(R)-4 \mathbf{c}$ & $0.125 \mathrm{M}$ & $-40^{\circ} \mathrm{C}$ & $>95 \%$ & $96: 4$ \\
4 & toluene & $(\mathrm{S})-\mathbf{4 b}$ & $0.125 \mathrm{M}$ & $-78^{\circ} \mathrm{C}$ & $>95 \%$ & $2.5: 97.5$ \\
5 & toluene & $(R)-4 \mathbf{c}$ & $0.125 \mathrm{M}$ & $-78^{\circ} \mathrm{C}$ & $>95 \%$ & $98: 2$ \\
6 & toluene & $(R)-4 \mathrm{c}$ & $0.063 \mathrm{M}$ & $-78^{\circ} \mathrm{C}$ & $>95 \%$ & $98: 2$ \\
7 & toluene & $(R)-4 \mathbf{c}$ & $0.042 \mathrm{M}$ & $-78^{\circ} \mathrm{C}$ & nd & $97.5: 2.5$ \\
\hline
\end{tabular}

[a] Determined by ${ }^{1} \mathrm{H}$ NMR; [b] determined by HPLC on chiral stationary phase.

Table S2. Catalyst loading screening in the ring opening of $1 \mathbf{a}$ with thiobenzoic acid.

\begin{tabular}{|c|c|c|c|c|c|}
\hline & & $\begin{array}{r}\mathbf{4 c}(\mathrm{X} \mathrm{mol}) \\
\mathrm{PhCOSH}(1.6 \\
\begin{array}{c}\text { toluene }(0.125 \\
\text { temperature }\end{array}\end{array}$ & $\underset{\text { M) }}{\stackrel{\text { quiv.) }}{\longrightarrow}}$ & $s-$ & \\
\hline entry & Catalyst & $x$ & temperature & Conv ${ }^{[a]}$ & $\mathrm{er}^{[\mathrm{b}]}$ \\
\hline 1 & $(R)-4 \mathrm{c}$ & $4 \mathrm{~mol} \%$ & r.t. & $>95 \%$ & $90: 10$ \\
\hline 2 & $(R)-4 \mathrm{c}$ & $1 \mathrm{~mol} \%$ & r.t. & $>95 \%$ & $90: 10$ \\
\hline 3 & $(R)-4 \mathrm{c}$ & $0.1 \mathrm{~mol} \%$ & r.t. & $>95 \%$ & $89.5: 10.5$ \\
\hline 4 & $(R)-4 \mathrm{c}$ & $0.001 \mathrm{~mol} \%$ & r.t. & $48 \%$ & $85: 15$ \\
\hline 5 & (R)-4c & $0.0001 \mathrm{~mol} \%$ & r.t. & $13 \%$ & $63: 37$ \\
\hline 6 & $(R)-4 c$ & $4 \mathrm{~mol} \%$ & $-40^{\circ} \mathrm{C}$ & $>95 \%$ & $96: 4$ \\
\hline 7 & $(R)-4 \mathrm{c}$ & $1 \mathrm{~mol} \%$ & $-40^{\circ} \mathrm{C}$ & $>95 \%$ & $96: 4$ \\
\hline 8 & $(R)-4 \mathrm{c}$ & $0.1 \mathrm{~mol} \%$ & $-40^{\circ} \mathrm{C}$ & $79 \%$ & $96: 4$ \\
\hline 9 & $(R)-4 \mathrm{c}$ & $0.01 \mathrm{~mol} \%$ & $-40^{\circ} \mathrm{C}$ & traces & - \\
\hline 10 & $(R)-4 \mathrm{c}$ & $4 \mathrm{~mol} \%$ & $-78^{\circ} \mathrm{C}$ & $>95 \%$ & $98: 2$ \\
\hline 11 & $(R)-4 \mathrm{c}$ & 2 mol\% & $-78^{\circ} \mathrm{C}$ & $>95 \%$ & $98: 2$ \\
\hline 12 & $(R)-4 \mathrm{c}$ & $1 \mathrm{~mol} \%$ & $-78^{\circ} \mathrm{C}$ & $89 \%$ & $97.5: 2.5$ \\
\hline
\end{tabular}

[a] Determined by ${ }^{1}$ H NMR; [b] determined by HPLC on chiral stationary phase. 


\section{Insights into the acyl-transfer step}

Experiment 1. This experiment was designed to evaluate the role of the phosphoric acid catalyst in the acyl-transfer step of the organocascade reaction.

Thioester product $3 \mathbf{a}(0.1 \mathrm{mmol}, 0.125 \mathrm{M})$ was dissolved in toluene and subjected to different acidic conditions (see table below) and the conversion to thiol $\mathbf{5 a}$ was monitored during time.

The outcome of the three reactions underlines the role of the phosphoric acid catalyst in the trans-esterification step. Intriguingly, the phosphoric acid catalyst is more active in this transformation in the absence of the thiocarboxylic acid.

Two possible scenarios may explain such outcome:

a) TRIP is the active catalyst and therefore the heterodimerization process interferes with the acyl transfer.

b) The heterodimer is the active catalyst in the mixture, but its activity is lower than that of free TRIP.

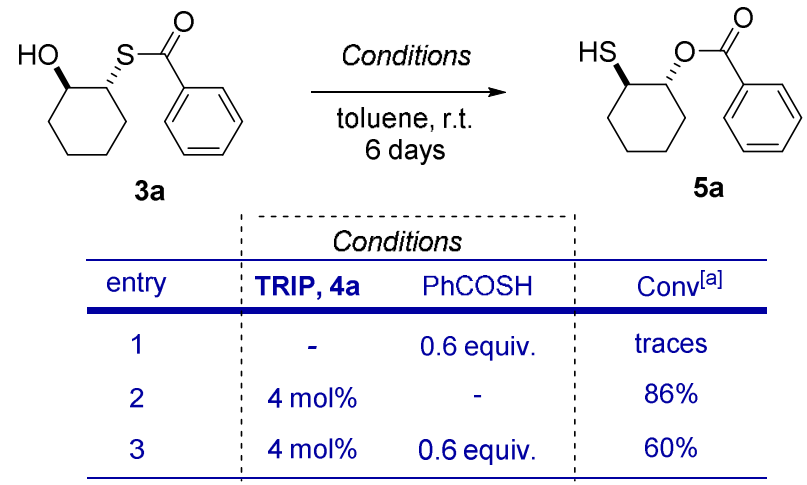

[a] Determined by ${ }^{1} \mathrm{H}-\mathrm{NMR}$ analysis

Experiment 2. Two reactions for the organocascade synthesis of compounds $\mathbf{5 d}$ and $\mathbf{5 f}$ were attempted. The reaction mixtures from the thiocarboxylysis step were heated to $40^{\circ} \mathrm{C}$ for several days but no conversion into the desired products was observed.

These experiments show that five membered-ring substrates do not undergo the acyl-transfer transformation and such outcome is in agreement with our proposed intramolecular reaction pathway. The requirement for a highly energetic trans-fused hetero-bicyclooctane intermediate Int-I presumably hampers the reaction.
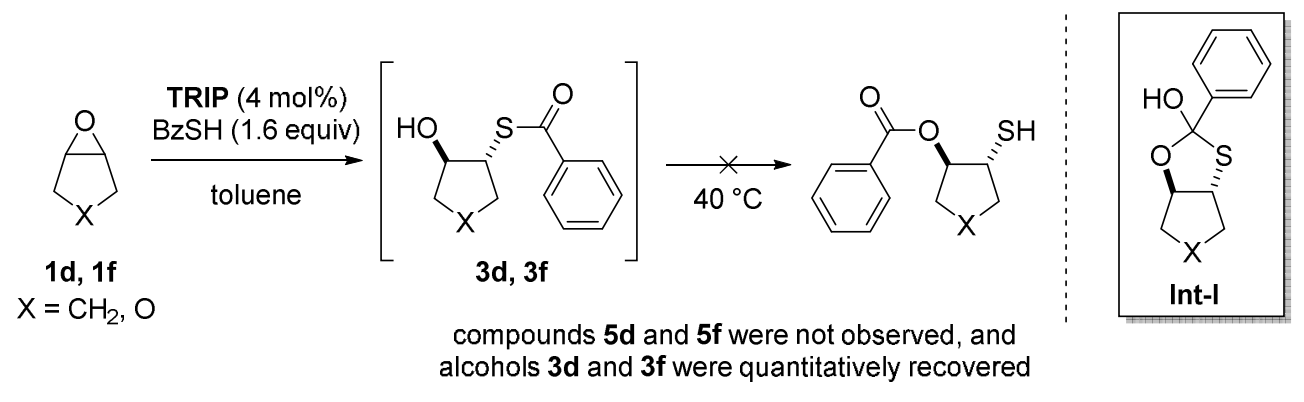


\section{Synthetic procedures and spectral data}

Thiobenzoic acid $\mathbf{2}$ and epoxides 1a, 1d, 1e, $\mathbf{1 h}$ and $\mathbf{1 k}$ were purchased from Sigma Aldrich. The phosphoric acid catalysts were synthesized following reported procedures. ${ }^{2}$

\subsection{Preparation of starting materials}

When not commercially available, the starting meso-epoxides for the ring opening reaction were synthesized via epoxidation from the corresponding alkene with $m$-CPBA following literature procedure. ${ }^{2}$

cis-1,2-Epoxy-4-cyclohexene (1b) -

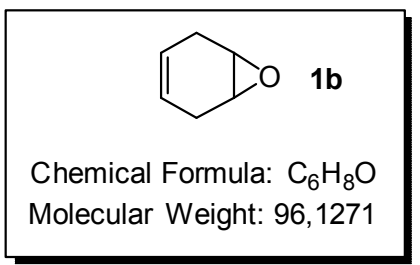

The reaction was performed on $5 \mathrm{mmol}$ scale and the product was isolated in $61 \%$ yield.

${ }^{1}$ H NMR $\left(500 \mathrm{MHz}, \mathrm{CDCl}_{3}\right): \delta 5.44(\mathrm{~s}, 2 \mathrm{H}), 3.25(\mathrm{~s}, 2 \mathrm{H}), 2.57(\mathrm{~d}, J=18.6 \mathrm{~Hz}, 2 \mathrm{H}), 2.44(\mathrm{~d}, J=18.5$ $\mathrm{Hz}, 2 \mathrm{H})$.

${ }^{13}$ C NMR (125 MHz, $\left.\mathrm{CDCl}_{3}\right): \delta 121.8,51.2,25.2$.

HRMS $(\mathrm{m} / \mathrm{z})$ calcd for $\mathrm{C}_{6} \mathrm{H}_{8} \mathrm{O}[\mathrm{M}]: 96.0575$, found: 96.0574 .

\section{cis-2,3-Epoxy-1,4-dihydronaphthalene (1c) -}

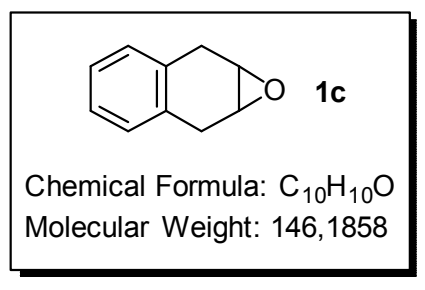

The reaction was performed on a $5 \mathrm{mmol}$ scale and the product was isolated in $75 \%$ yield.

${ }^{1} \mathbf{H}$ NMR $\left(500 \mathrm{MHz}, \mathrm{CDCl}_{3}\right): \delta 7.17-7.11(\mathrm{~m}, 2 \mathrm{H}), 7.08-7.01(\mathrm{~m}, 2 \mathrm{H}), 3.48(\mathrm{~s}, 2 \mathrm{H}), 3.32(\mathrm{~d}, J=17.1$ $\mathrm{Hz}, 2 \mathrm{H}), 3.20(\mathrm{~d}, J=17.6 \mathrm{~Hz}, 2 \mathrm{H})$.

${ }^{13}$ C NMR (125 MHz, $\left.\mathrm{CDCl}_{3}\right): \delta 131.7,129.5,126.8,52.0,29.9$.

HRMS $(m / z)$ calcd for $\mathrm{C}_{10} \mathrm{H}_{10} \mathrm{O}[\mathrm{M}]: 146.0732$, found: 146.0731 .

${ }^{2}$ M. R. Monaco, S. Prévost, B. List Angew. Chem. Int. Ed. 2014, 53, 8142. 
6-Oxa-3-oxa-bicyclo[3.1.0]hexane (1f) -

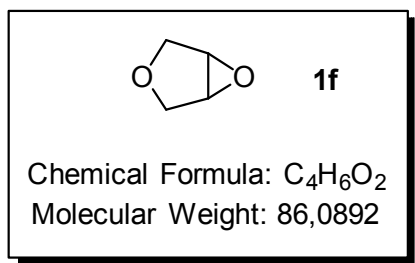

The reaction was performed on a $5 \mathrm{mmol}$ scale and the product was isolated in $58 \%$ yield.

${ }^{1} \mathbf{H}$ NMR (500 MHz, $\left.\mathrm{CDCl}_{3}\right): \delta 4.02(\mathrm{~d}, J=10.5 \mathrm{~Hz}, 2 \mathrm{H}), 3.78(\mathrm{~s}, 2 \mathrm{H}), 3.65(\mathrm{~d}, J=10.5 \mathrm{~Hz}, 2 \mathrm{H})$.

${ }^{13}$ C NMR (125 MHz, $\left.\mathrm{CDCl}_{3}\right): \delta 67.5,56.1$.

HRMS $(m / z)$ calcd for $\mathrm{C}_{4} \mathrm{H}_{6} \mathrm{O}_{2}[\mathrm{M}]: 86.0368$, found: 86.0368 .

\section{Benzyl 6-oxa-3-azabicyclo[3.1.0]hexane-3-carboxylate (1g) -}

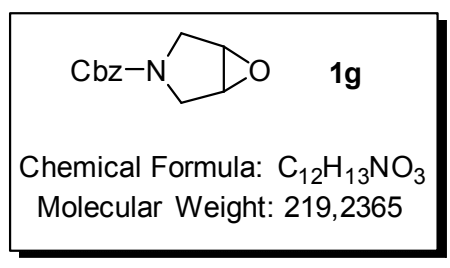

The reaction was performed on a $2.5 \mathrm{mmol}$ scale and the product was isolated in $79 \%$ yield.

${ }^{1} \mathbf{H}$ NMR $\left(500 \mathrm{MHz}, \mathrm{CDCl}_{3}\right): \delta$ 7.39-7.28 (m, 5H), $5.13(\mathrm{~d}, J=12,4 \mathrm{~Hz}, 1 \mathrm{H}), 5.09(\mathrm{~d}, J=12,4 \mathrm{~Hz}$, $1 \mathrm{H}), 3.87$ (dd, $J=24.5, J=12.8 \mathrm{~Hz}, 2 \mathrm{H}), 3.71-3.66(\mathrm{~m}, 2 \mathrm{H}), 3.39$ (ddd, $J=7.9, J=4.8, J=0.8 \mathrm{~Hz}$, $2 \mathrm{H})$.

${ }^{13}$ C NMR (125 MHz, $\left.\mathrm{CDCl}_{3}\right): \delta 155.5,136.8,128.7,128.3,128.2,67.2,55.7,55.2,47.6,47.4$.

HRMS $(m / z)$ calcd for $\mathrm{C}_{12} \mathrm{H}_{13} \mathrm{NO}_{3}[\mathrm{M}]^{+\mathrm{Na}}: 242.0788$, found: 242.0785 .

cis-4,5-Epoxyoctane (1i) -

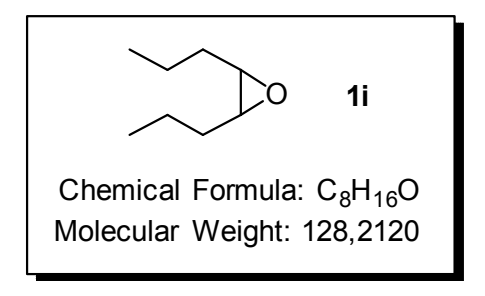

The reaction was performed on a $5 \mathrm{mmol}$ scale and the product was isolated in $63 \%$ yield.

${ }^{1} \mathbf{H}$ NMR $\left(500 \mathrm{MHz}, \mathrm{CDCl}_{3}\right): \delta 2.94-2.89(\mathrm{~m}, 2 \mathrm{H}), 1.59-1.42(\mathrm{~m}, 8 \mathrm{H}), 0.98(\mathrm{t}, J=7.2 \mathrm{~Hz}, 6 \mathrm{H})$.

${ }^{13}$ C NMR (125 MHz, $\left.\mathrm{CDCl}_{3}\right): \delta 57.2,30.1,20.1,14.3$.

HRMS $(\mathrm{m} / \mathrm{z})$ calcd for $\mathrm{C}_{8} \mathrm{H}_{16} \mathrm{O}[\mathrm{M}]:$ : 28.1201 , found: 128.1202 . 
cis-3,4-Epoxyhexane (1j) -

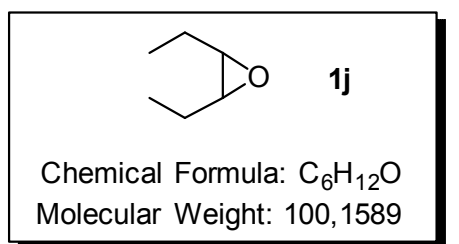

The reaction was performed on a $5 \mathrm{mmol}$ scale and the product was isolated in $37 \%$ yield.

${ }^{1} \mathbf{H}$ NMR $\left(500 \mathrm{MHz}, \mathrm{CDCl}_{3}\right): \delta 2.91-2.85(\mathrm{~m}, 2 \mathrm{H}), 1.63-1.44(\mathrm{~m}$, $4 \mathrm{H}), 1.04(\mathrm{t}, J=7.5 \mathrm{~Hz}, 6 \mathrm{H})$.

${ }^{13}$ C NMR (125 MHz, $\left.\mathrm{CDCl}_{3}\right): \delta 58.7,21.2,10.8$.

HRMS $(\mathrm{m} / \mathrm{z})$ calcd for $\mathrm{C}_{6} \mathrm{H}_{12} \mathrm{O}$ [M]: 100.0888, found: 100.0887 . 


\subsection{Asymmetric ring opening reaction}

\subsubsection{General procedure for the thiocarboxylysis of meso-epoxides}<smiles>[R]C1OC1[O]</smiles>

$\mathrm{O}_{\mathrm{R}^{1}}^{\mathrm{SH}}$

2a-b

1a-k $\underset{\text { Toluene }(0.125 \mathrm{M})}{\stackrel{\text { Cat }(4 \mathrm{~mol} \%)}{\longrightarrow}}$

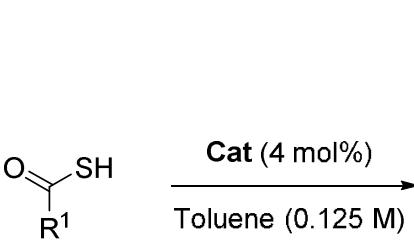

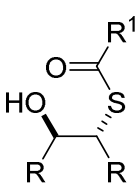

3a-m

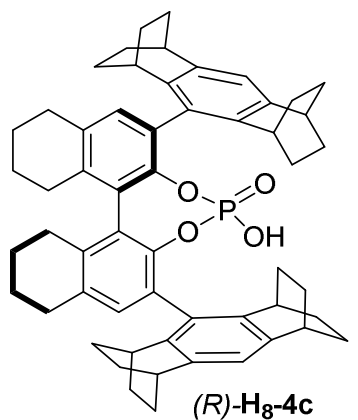

In a screw-cap vial, epoxide $1(0.1 \mathrm{mmol})$ was dissolved in $0.4 \mathrm{~mL}$ of toluene and was cooled to the reaction temperature. Then a pre-cooled solution of thiocarboxylic acid $2(0.16$ mmol, 1.6 equiv.) and catalyst $4 \mathbf{c}(4 \mathrm{~mol} \%)$ in $0.4 \mathrm{~mL}$ of toluene was added and the reaction was stirred for 2-5 days. Next, $1 \mathrm{~mL}$ of MTBE was added to the reaction and the cold mixture was directly purified by flash column chromatography (eluant: mixtures hexane/MTBE). The enantiomeric ratios of products 3 were measured by HPLC on a chiral stationary phase.

\subsubsection{General procedure for the organocascade synthesis of thiols 5a-n}<smiles>[R]C1OC1[NH-]</smiles>

2a-b

1a-k
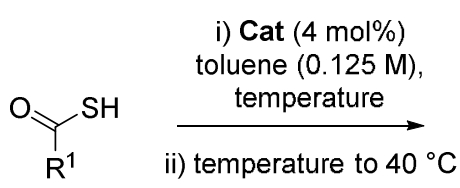

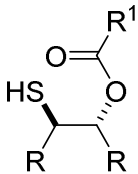

5a-n

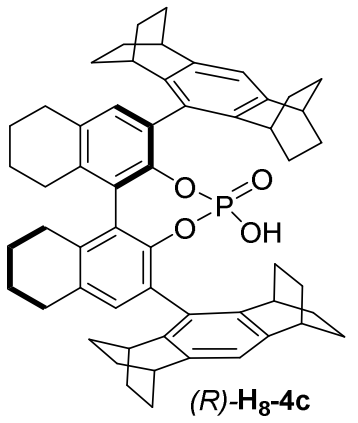

In a screw-cap vial, epoxide $1(0.1 \mathrm{mmol})$ was dissolved in $0.4 \mathrm{~mL}$ of toluene and was cooled to the reaction temperature. Then a pre-cooled solution of thiocarboxylic acid $2(0.16$ mmol, 1.6 equiv.) and catalyst $4 \mathbf{c}(4 \mathrm{~mol} \%)$ in $0.4 \mathrm{~mL}$ of toluene was added and the reaction was stirred for 2-5 days. Next, the temperature was raised to room temperature and subsequently to $40{ }^{\circ} \mathrm{C}$ and the mixture was stirred for $1-2$ days. The reaction mixture was directly purified by flash column chromatography (eluant: mixtures hexane/MTBE). The enantiomeric ratios of products were measured by HPLC on a chiral stationary phase.

NOTE: The racemic samples were prepared using as catalyst a 1:1 mixture of $(S)$-TRIP and $(R)$-TRIP. 


\subsubsection{Thiocarboxylysis products}

3a - S-((1R,2R)-2-hydroxycyclohexyl) benzothioate -

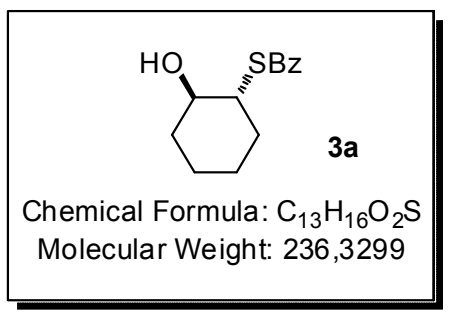

The reaction was performed at $-78^{\circ} \mathrm{C}$ and the product was isolated as a white solid in $95 \%$ yield and e.r. $=98: 2$.

${ }^{1} \mathbf{H}$ NMR $\left(300 \mathrm{MHz}, \mathrm{CD}_{2} \mathrm{Cl}_{2}\right): \delta$ 8.00-7.92 (m, 2H), 7.65-7.55 (m, 1H), 7.52-7.42 (m, 2H), 3.70-3.42 (m, 2H), 2.37 (d, 1H, J=4.6 Hz), 2.20-2.05 (m, 2H), 1.85-1.68 (m, 2H), 1.50-1.20 (m, 4H).

${ }^{13} \mathrm{C}$ NMR (75 MHz, $\left.\mathrm{CD}_{2} \mathrm{Cl}_{2}\right): \delta 192.6,137.5,133.9,129.0,127.7,73.4,50.7,35.6,32.6,26.3,24.6$.

HRMS $(m / z)$ calcd for $\mathrm{C}_{13} \mathrm{H}_{16} \mathrm{O}_{2} \mathrm{SNa}[\mathrm{M}]^{+\mathrm{Na}}: 259.0763$, found: 259.0762 .

$[\alpha]_{\mathbf{D}}{ }^{25}:-39.6^{\circ}\left(c=1.050, \mathrm{CHCl}_{3}\right)$.

The enantiomeric ratio was determined by HPLC analysis using Daicel Chiralcel AD-3 column: $n \mathrm{Hept}: i \operatorname{PrOH}=95: 5$, flow rate $1.0 \mathrm{~mL} / \mathrm{min}, \tau_{1}=13.0 \mathrm{~min}, \tau_{2}=17.5 \mathrm{~min}$.

$3 \mathrm{~b}-S-((1 R, 6 R)-6$-hydroxycyclohex-3-en-1-yl) benzothioate -

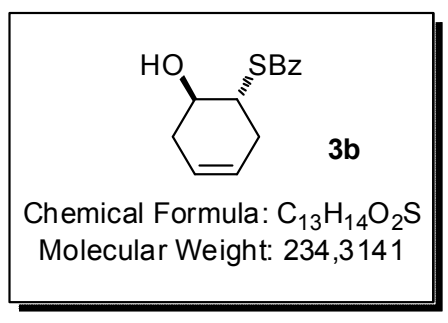

The reaction was performed at $-40^{\circ} \mathrm{C}$ and the product was isolated as a colorless liquid in $99 \%$ yield and e.r. $=96.5: 3.5$.

${ }^{1} \mathbf{H}$ NMR $\left(300 \mathrm{MHz}, \mathrm{CDCl}_{3}\right): \delta$ 8.05-7.92 (m, 2H), 7.64-7.55 (m, 1H), 7.50-7.42 (m, 2H), 5.75-5.60 (m, 2H), 4.05-3.82 (m, 2H), 2.78-2.55 (m, 2H), 2.45-2.18 (m, 3H).

${ }^{13}$ C NMR (75 MHz, $\left.\mathrm{CDCl}_{3}\right): \delta 192.5,137.1,133.8,128.8,127.6,125.3,125.0,69.6,45.8,34.1,31.2$.

HRMS $(\mathrm{m} / z)$ calcd for $\mathrm{C}_{13} \mathrm{H}_{14} \mathrm{O}_{2} \mathrm{SNa}[\mathrm{M}]^{+\mathrm{Na}}: 257.0607$, found: 257.0606 .

$[\alpha]_{\mathbf{D}}{ }^{25}:-43.5^{\circ}\left(c=1.200, \mathrm{CHCl}_{3}\right)$.

The enantiomeric ratio was determined by HPLC analysis using Daicel Chiralcel AD-3 column: $n$ Hept: $i \mathrm{PrOH}=95: 5$, flow rate $1.0 \mathrm{~mL} / \mathrm{min}, \tau_{1}=13.4 \mathrm{~min}, \tau_{2}=15.1 \mathrm{~min}$. 


\section{$3 \mathrm{c}-S$-((2R,3R)-3-hydroxy-1,2,3,4-tetrahydronaphthalen-2-yl) benzothioate -}

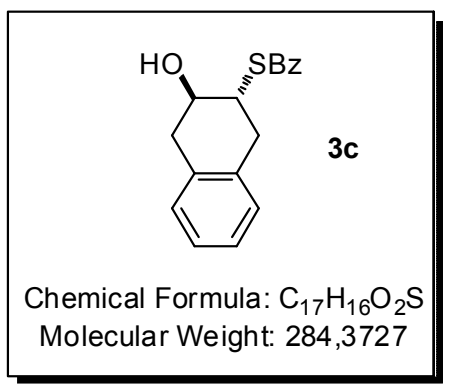

The reaction was performed at $-40^{\circ} \mathrm{C}$ and the product was isolated as a colorless liquid in $86 \%$ yield and e.r. $=96.5: 3.5$.

${ }^{1} \mathbf{H}$ NMR $\left(300 \mathrm{MHz}, \mathrm{CD}_{2} \mathrm{Cl}_{2}\right): \delta$ 8.05-7.92 (m, 2H), 7.68-7.56 (m, $\left.1 \mathrm{H}\right)$, 7.52-7.42 (m, 2H), 7.22-7.08 (m, 4H), 4.20-4.05 (m, 2H), 3.50-3.22 (m, 2H), 3.10-2.88 (m, 2H), 2.48 (bd, $J=3.5 \mathrm{~Hz}, 1 \mathrm{H})$.

${ }^{13}$ C NMR (75 MHz, $\left.\mathrm{CD}_{2} \mathrm{Cl}_{2}\right): \delta 192.1,137.3,134.4,134.2,134.0,129.5,129.1,128.6,127.6,126.8$, 126.5, 70.1, 46.4, 37.5, 34.2.

HRMS $(m / z)$ calcd for $\mathrm{C}_{17} \mathrm{H}_{16} \mathrm{O}_{2} \mathrm{SNa}[\mathrm{M}]^{+\mathrm{Na}}: 307.0763$, found: 307.0763 .

$[\alpha]_{\mathbf{D}}{ }^{25}:-64.8^{\circ}\left(c=1.225, \mathrm{CHCl}_{3}\right)$.

The enantiomeric ratio was determined by HPLC analysis using Daicel Chiralcel AD-3 column: $n \mathrm{Hept}: i \mathrm{PrOH}=95: 5$, flow rate $1.0 \mathrm{~mL} / \mathrm{min}, \tau_{1}=22.7 \mathrm{~min}, \tau_{2}=25.9 \mathrm{~min}$.

\section{3d - S-((1R,2R)-2-hydroxycyclopentyl) benzothioate -}

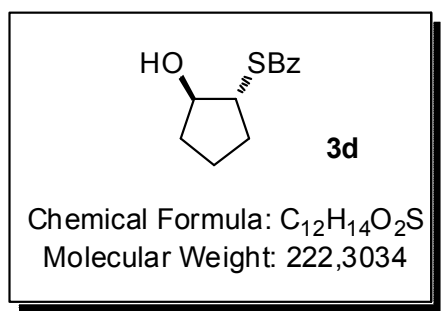

The reaction was performed at $-40^{\circ} \mathrm{C}$ and the product was isolated as a colorless liquid in 99\% yield and e.r. $=97: 3$.

${ }^{1} \mathbf{H}$ NMR $\left(300 \mathrm{MHz}, \mathrm{CD}_{2} \mathrm{Cl}_{2}\right): \delta$ 8.00-7.90 (m, 2H), 7.65-7.54 (m, 1H), 7.52-7.43 (m, 2H), 4.25-4.15 (m, 1H), 3.72 (td, $J=4.5 \mathrm{~Hz} J=8.1 \mathrm{~Hz}, 1 \mathrm{H}), 2.91$ (bs, $1 \mathrm{H}), 2.40-2.22(\mathrm{~m}, 1 \mathrm{H}), 2.10-1.58(\mathrm{~m}, 5 \mathrm{H})$.

${ }^{13} \mathbf{C}$ NMR (75 MHz, $\left.\mathrm{CD}_{2} \mathrm{Cl}_{2}\right): \delta 194.5,137.4,133.9,129.1,127.6,80.9,51.4,34.3,30.8,23.5$.

HRMS $(\mathrm{m} / \mathrm{z})$ calcd for $\mathrm{C}_{12} \mathrm{H}_{14} \mathrm{O}_{2} \mathrm{SNa}[\mathrm{M}]^{+\mathrm{Na}}: 245.0607$, found: 245.0607 .

$[\alpha]_{\mathbf{D}}^{25}:+37.5^{\circ}\left(c=1.100, \mathrm{CHCl}_{3}\right)$.

The enantiomeric ratio was determined by HPLC analysis using Daicel Chiralcel AD-3 column: $n$ Hept: $i \mathrm{PrOH}=95: 5$, flow rate $1.0 \mathrm{~mL} / \mathrm{min}, \tau_{1}=10.5 \mathrm{~min}, \tau_{2}=11.8 \mathrm{~min}$. 


\section{$3 \mathrm{e}-S-((1 R, 2 R)-2$-hydroxycycloheptyl) benzothioate -}

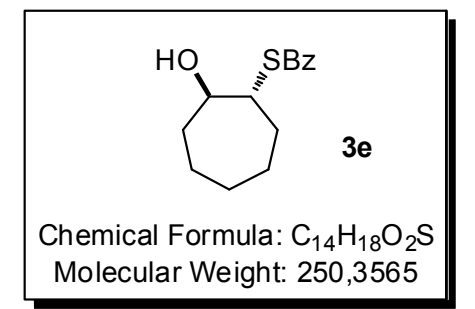

The reaction was performed at $-40^{\circ} \mathrm{C}$ and the product was isolated as white solid in $86 \%$ yield and e.r. $=96.5: 3.5$.

${ }^{1} \mathbf{H}$ NMR $\left(300 \mathrm{MHz}, \mathrm{CD}_{2} \mathrm{Cl}_{2}\right): \delta$ 8.04-7.90 (m, 2H), 7.65-7.56 (m, 1H), 7.52-7.42 (m, 2H), 3.95-3.70 (m, 2H), 2.48 (bs, 1H), 2.15-1.40 (m, 10H).

${ }^{13} \mathrm{C}$ NMR $\left(75 \mathrm{MHz}, \mathrm{CD}_{2} \mathrm{Cl}_{2}\right): \delta 192.9,137.6,133.8,129.0,127.6,76.3,53.6,35.3,32.1,28.7,26.9$, 22.7 .

HRMS $(m / z)$ calcd for $\mathrm{C}_{14} \mathrm{H}_{18} \mathrm{O}_{2} \mathrm{SNa}[\mathrm{M}]^{+\mathrm{Na}}: 273.0920$, found: 273.0919 .

$[\alpha]_{\mathbf{D}}{ }^{25}:-28.2^{\circ}\left(c=0.985, \mathrm{CHCl}_{3}\right)$.

The enantiomeric ratio was determined by HPLC analysis using Daicel Chiralcel AD-3 column: $n$ Hept: $i \operatorname{PrOH}=95: 5$, flow rate $1.0 \mathrm{~mL} / \mathrm{min}, \tau_{1}=13.4 \mathrm{~min}, \tau_{2}=16.8 \mathrm{~min}$.

\section{3f - S-((3R,4R)-4-hydroxytetrahydrofuran-3-yl) benzothioate -}

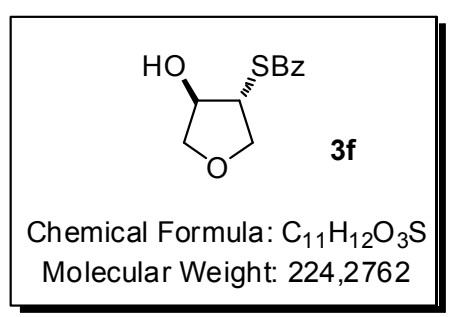

The reaction was performed at $-10^{\circ} \mathrm{C}$ and the product was isolated as a colorless liquid in $78 \%$ yield and e.r. $=95.5: 4.5$.

${ }^{1} \mathbf{H}$ NMR $\left(300 \mathrm{MHz}, \mathrm{CD}_{2} \mathrm{Cl}_{2}\right.$ ): $\delta$ 8.00-7.90 (m, 2H), 7.68-7.58 (m, 1H), 7.55-7.45 (m, 2H), 4.48-4.32 (m, 2H), 4.08-3.92 (m, 2H), 3.80-3.70 (dd, $J=9.8 \mathrm{~Hz} J=4.1 \mathrm{~Hz}, 2 \mathrm{H}), 2.78$ (bd, $J=3.4 \mathrm{~Hz}, 1 \mathrm{H})$.

${ }^{13}$ C NMR (75 MHz, $\left.\mathrm{CD}_{2} \mathrm{Cl}_{2}\right): \delta 192.8,137.0,134.2,129.2,127.6,79.3,74.6,71.3,50.7$.

HRMS $(m / z)$ calcd for $\mathrm{C}_{11} \mathrm{H}_{12} \mathrm{O}_{3} \mathrm{SNa}[\mathrm{M}]^{+\mathrm{Na}}: 247.0399$, found: 247.0340 .

$[\alpha]_{\mathbf{D}}^{25}:+11.5^{\circ}\left(c=0.850, \mathrm{CHCl}_{3}\right)$.

The enantiomeric ratio was determined by HPLC analysis using Daicel Chiralcel AD-3 column: $n$ Hept: $i \mathrm{PrOH}=97: 3$, flow rate $1.0 \mathrm{~mL} / \mathrm{min}, \tau_{1}=24.7 \mathrm{~min}, \tau_{2}=26.6 \mathrm{~min}$. 


\section{$3 g$ - (3R,4R)-benzyl 3-(benzoylthio)-4-hydroxypyrrolidine-1-}

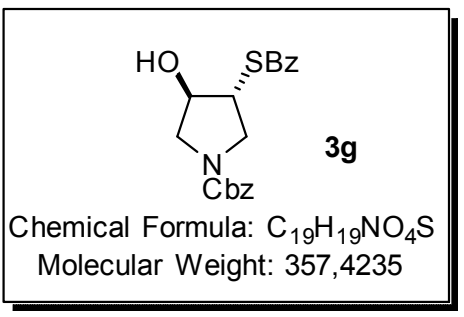

\section{carboxylate -}

The reaction was performed at $-10^{\circ} \mathrm{C}$ and the product was isolated as a colorless liquid in $63 \%$ yield and e.r. $=95.5: 4.5$.

${ }^{1} \mathbf{H}$ NMR $\left(300 \mathrm{MHz}, \mathrm{CDCl}_{3}\right): \delta$ 7.95-7.88 (m, 2H), 7.65-7.57 (m, 1H), 7.52-7.43 (m, 2H), 7.42-7.27 (m, 5H), 5.17 (s, 2H), 4.44-4.35 (m, 1H), 4.18-4.00 (m, 2H), 3.92-3.36 (m, 3H), 2.80 (bs, 1H).

${ }^{13}$ C NMR (75 MHz, $\left.\mathrm{CDCl}_{3}\right): \delta 191.0,154.9,136.8,136.6,134.2,129.0,128.7,128.3,128.2,127.6$, $76.5,75.3,67.3,52.7,52.2,49.1,48.6$ (additional peaks due to the presence of rotamers).

HRMS $(m / z)$ calcd for $\mathrm{C}_{19} \mathrm{H}_{19} \mathrm{NO}_{4} \mathrm{SNa}[\mathrm{M}]^{+\mathrm{Na}}: 380.0927$, found: 380.0928 .

$[\alpha]_{\mathbf{D}}{ }^{25}:+53.4^{\circ}\left(c=0.700, \mathrm{CHCl}_{3}\right)$.

The enantiomeric ratio was determined by HPLC analysis using Daicel Chiralcel OD-3 column: $n \mathrm{Hept}: i \mathrm{PrOH}=85: 15$, flow rate $1.0 \mathrm{~mL} / \mathrm{min}, \tau_{1}=12.2 \mathrm{~min}, \tau_{2}=15.7 \mathrm{~min}$.

\section{$3 \mathrm{~h}-S-((2 R, 3 R)-3-\mathrm{hydroxybutan}-2$-yl) benzothioate -}

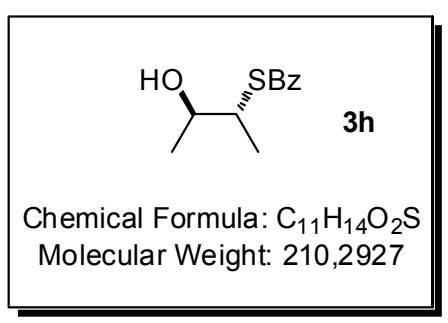

The reaction was performed at $-40^{\circ} \mathrm{C}$ and the product was isolated as a colorless liquid in $99 \%$ yield and e.r. $=97: 3$.

${ }^{1} \mathbf{H}$ NMR $\left(300 \mathrm{MHz}, \mathrm{CD}_{2} \mathrm{Cl}_{2}\right): \delta$ 8.10-7.95 (m, 2H), 7.65-7.52 (m, 1H), 7.52-7.42 (m, 2H), 4.00-3.89 (m, 1H), 3.83 (dq, $J=4.6 \mathrm{~Hz} J=7.0 \mathrm{~Hz}, 1 \mathrm{H}), 1.96$ (bs, 1H), 1.43 (d, $J=7.2 \mathrm{~Hz}, 3 \mathrm{H}), 1.26$ (d, $J=6.2$ $\mathrm{Hz}, 3 \mathrm{H})$.

${ }^{13}$ C NMR (75 MHz, $\left.\mathrm{CD}_{2} \mathrm{Cl}_{2}\right): \delta 191.9,137.7,133.8,129.0,127.6,71.1,46.8,20.8,18.0$.

HRMS $(m / z)$ calcd for $\mathrm{C}_{11} \mathrm{H}_{14} \mathrm{O}_{2} \mathrm{SNa}[\mathrm{M}]^{+\mathrm{Na}}: 233.0607$, found: 233.0606 .

$[\alpha]_{\mathbf{D}}{ }^{25}:-26.6^{\circ}\left(c=1.175, \mathrm{CHCl}_{3}\right)$.

The enantiomeric ratio was determined by HPLC analysis using Daicel Chiralcel AD-3 column: $n$ Hept: $i \mathrm{PrOH}=95: 5$, flow rate $1.0 \mathrm{~mL} / \mathrm{min}, \tau_{1}=7.9 \mathrm{~min}, \tau_{2}=8.9 \mathrm{~min}$. 


\section{$3 \mathrm{i}-S-((4 R, 5 R)-5-h y d r o x y o c t a n-4-y l)$ benzothioate -}

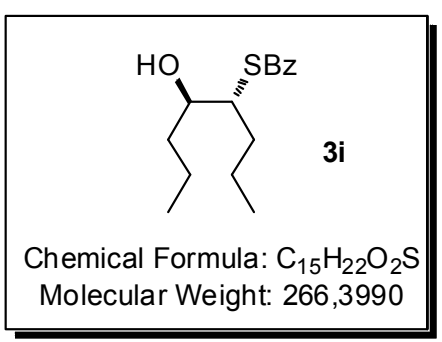

The reaction was performed at $-40^{\circ} \mathrm{C}$ and the product was isolated as a colorless liquid in $82 \%$ yield and e.r. $=95: 5$.

${ }^{1} \mathbf{H}$ NMR $\left(300 \mathrm{MHz}, \mathrm{CD}_{2} \mathrm{Cl}_{2}\right): \delta$ 8.02-7.96 (m, 2H), 7.64-7.55 (m, $\left.1 \mathrm{H}\right)$, 7.52-7.43 (m, 2H), 3.88-3.76 (m, 2H), 1.90-1.30 (m, 9H), 0.98-0.88 (m, 6H).

${ }^{13} \mathbf{C}$ NMR $\left(75 \mathrm{MHz}, \mathrm{CD}_{2} \mathrm{Cl}_{2}\right): \delta 192.0,137.6,133.7,128.9,127.6,73.9,50.8,37.5,34.9,20.8,19.5$, 14.1, 14.0.

HRMS $(m / z)$ calcd for $\mathrm{C}_{15} \mathrm{H}_{22} \mathrm{O}_{2} \mathrm{SNa}[\mathrm{M}]^{+\mathrm{Na}}: 289.1232$, found: 289.1232 .

$[\alpha]_{\mathbf{D}}^{25}:+19.6^{\circ}\left(c=1.100, \mathrm{CHCl}_{3}\right)$.

The enantiomeric ratio was determined by HPLC analysis using Daicel Chiralcel OJ-H column: $n \mathrm{Hept}: \mathrm{PrOH}=98: 2$, flow rate $1.0 \mathrm{~mL} / \mathrm{min}, \tau_{1}=11.0 \mathrm{~min}, \tau_{2}=12.1 \mathrm{~min}$.

\section{$3 \mathrm{j}-S-((3 R, 4 R)-4-h y d r o x y h e x a n-3-y l)$ benzothioate -}

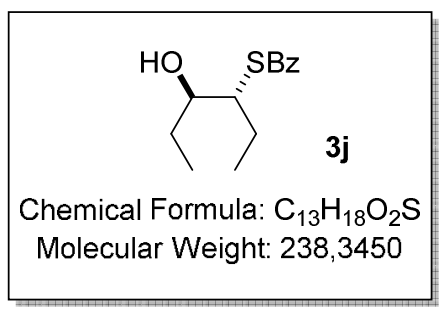

The reaction was performed at $-40^{\circ} \mathrm{C}$ and the product was isolated as a colorless liquid in $94 \%$ yield and e.r. $=95.5: 4.5$.

${ }^{1} \mathbf{H}$ NMR $\left(300 \mathrm{MHz}, \mathrm{CD}_{2} \mathrm{Cl}_{2}\right): \delta$ 8.04-7.95 (m, 2H), 7.64-7.55 (m, 1H), 7.52-7.43 (m, 2H), 3.83-3.70 (m, 2H), 2.00-1.40 (m, 5H), 1.04 (t, $J=7.4 \mathrm{~Hz}, 3 \mathrm{H}), 0.98$ (t, $J=7.4 \mathrm{~Hz}, 3 \mathrm{H})$.

${ }^{13}$ C NMR (75 MHz, $\mathrm{CD}_{2} \mathrm{Cl}_{2}$ ): $\delta$ 192.2, 137.7, 133.7, 129.0, 127.7, 75.4, 52.5, 28.4, 26.1, 12.0, 10.4.

HRMS $(\mathrm{m} / \mathrm{z})$ calcd for $\mathrm{C}_{13} \mathrm{H}_{18} \mathrm{O}_{2} \mathrm{SNa}[\mathrm{M}]^{+\mathrm{Na}}: 261.0920$, found: 261.0921 .

$[\alpha]_{\mathbf{D}}^{25}:-17.9^{\circ}\left(c=1.050, \mathrm{CHCl}_{3}\right)$.

The enantiomeric ratio was determined by HPLC analysis using Daicel Chiralcel AD-3 column: $n$ Hept: $i \mathrm{PrOH}=95: 5$, flow rate $1.0 \mathrm{~mL} / \mathrm{min}, \tau_{1}=8.0 \mathrm{~min}, \tau_{2}=8.6 \mathrm{~min}$. 
$3 \mathrm{k}-S$-((1R,2R)-2-hydroxy-1,2-diphenylethyl) benzothioate -

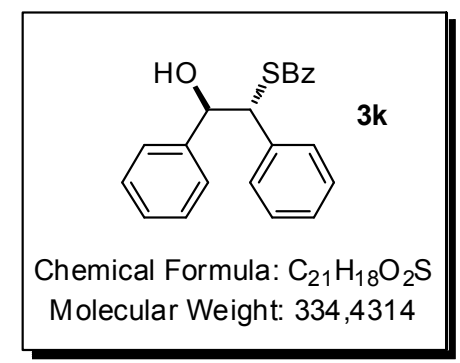

The reaction was performed at $-10^{\circ} \mathrm{C}$ and the product was isolated as a mixture $8.5: 1$ of compound $\mathbf{3 k}$ and $\mathbf{5 k}$ (white solid) in $72 \%$ yield and e.r. $. \mathbf{k} \mathbf{k}=89: 11$, e.r. $. \mathbf{k} \mathbf{k}=87: 13$.

${ }^{1} \mathbf{H}$ NMR $\left(300 \mathrm{MHz}, \mathrm{CD}_{2} \mathrm{Cl}_{2}\right): \delta 8.08-8.00\left(\mathrm{~m}, 2 \mathrm{H}_{5 \mathrm{k}}\right), 7.90-7.80\left(\mathrm{~m}, 2 \mathrm{H}_{3 \mathrm{k}}\right), 7.58-7.45\left(\mathrm{~m}, 1 \mathrm{H}_{3 \mathrm{k}}+1 \mathrm{H}_{5 \mathrm{k}}\right)$, 7.45-7.32(m, $\left.2 \mathrm{H}_{3 \mathrm{k}}+2 \mathrm{H}_{5 \mathrm{k}}\right), 7.26-7.08\left(\mathrm{~m}, 10 \mathrm{H}_{3 \mathrm{k}}+10 \mathrm{H}_{5 \mathrm{k}}\right), 6.13\left(\mathrm{~d}, J=8.5 \mathrm{~Hz}, 1 \mathrm{H}_{5 \mathrm{k}}\right), \quad 5.08-4.98(\mathrm{~m}$, $\left.2 \mathrm{H}_{3 \mathrm{k}}\right), 4.50\left(\mathrm{dd}, J=8.5 \mathrm{~Hz} J=5.2 \mathrm{~Hz}, 1 \mathrm{H}_{5 \mathrm{k}}\right), 2.72\left(\mathrm{bs}, 1 \mathrm{H}_{3 \mathrm{k}}\right), 2.28\left(\mathrm{~d}, J=5.2 \mathrm{~Hz}, 1 \mathrm{H}_{5 \mathrm{k}}\right)$.

${ }^{13}$ C NMR (75 MHz, $\mathrm{CD}_{2} \mathrm{Cl}_{2}$, compound 3k): $\delta$ 191.2, 141.7, 139.8, 137.2, 134.0, 129.1, 129.0, 128.8, $128.4,128.2,127.9,127.7,127.1,77.6,56.7$.

HRMS $(m / z)$ calcd for $\mathrm{C}_{21} \mathrm{H}_{18} \mathrm{O}_{2} \mathrm{SNa}[\mathrm{M}]^{+\mathrm{Na}}: 357.0920$, found: 341.0919 .

The enantiomeric ratio was determined by HPLC analysis using Daicel Chiralcel AD-3 column: $n \mathrm{Hept}: i \mathrm{PrOH}=85: 15$, flow rate $1.0 \mathrm{~mL} / \mathrm{min}, \tau_{1}=13.2 \mathrm{~min}, \tau_{2}=15.4 \mathrm{~min}$.

\section{$31-S-((1 R, 2 R)-2-h y d r o x y c y c l o h e x y l)$ ethanethioate-}

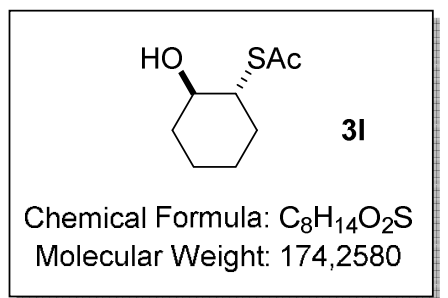

The reaction was performed at $-40^{\circ} \mathrm{C}$ and the product was isolated as a mixture 15:1 of compound $\mathbf{3 l}$ and $\mathbf{5 l}$ in $75 \%$ yield and e.r. ${ }_{3 \mathbf{l}}=95: 5$.

${ }^{1} \mathbf{H}$ NMR $\left(500 \mathrm{MHz}, \mathrm{CD}_{2} \mathrm{Cl}_{2}\right): \delta$ 3.35-3.20 (m, 2H), $2.25(\mathrm{~s}, 3 \mathrm{H}), 2.17(\mathrm{~d}, J=4.5 \mathrm{~Hz}, 1 \mathrm{H}), 2.05-1.86$ (m, 2H), 1.70-1.55 (m, 2H), 1.40-1.15 (m, 4H).

${ }^{13}$ C NMR (125 MHz, $\left.\mathrm{CD}_{2} \mathrm{Cl}_{2}\right): \delta$ 196.8, 73.2, 50.6, 35.4, 32.4, 31.1, 26.2, 24.5.

HRMS $(m / z)$ calcd for $\mathrm{C}_{8} \mathrm{H}_{14} \mathrm{O}_{2} \mathrm{SNa}[\mathrm{M}]^{+\mathrm{Na}}: 197.0607$, found: 197.0608 .

The enantiomeric ratio was determined by GC analysis using BGB-177/BG-15 column $30 \mathrm{~m}$ (60 min at $140{ }^{\circ} \mathrm{C}, 10^{\circ} \mathrm{C} / \mathrm{min}$ until $220^{\circ} \mathrm{C}, 3$ min at $220^{\circ} \mathrm{C}, 0.4$ bar $\left.\mathrm{H}_{2}\right), \tau_{1}=24.7 \mathrm{~min}, \tau_{2}=25.4 \mathrm{~min}$. 


\section{$3 \mathrm{~m}-S-((1 R, 2 R)-2-m e r c a p t o c y c l o p e n t y l$ acetate-}

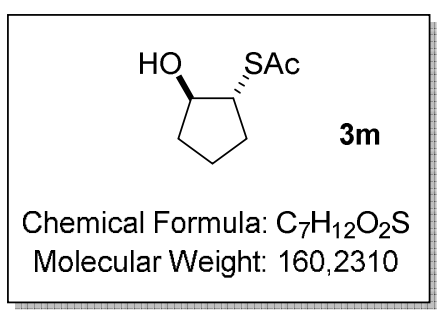

The reaction was performed at $-10^{\circ} \mathrm{C}$ and the product was isolated as colorless liquid in 63\% yield and e.r. $=93.5: 6.5$.

${ }^{1} \mathbf{H}$ NMR (500 MHz, $\mathrm{CD}_{2} \mathrm{Cl}_{2}$ ): $\delta$ 4.05-3.95 (m, 1H), $3.43(\mathrm{td}, J=8.0 \mathrm{~Hz}, J=4.5 \mathrm{~Hz}, 1 \mathrm{H}), 2.73$ (bs, $1 \mathrm{H}), 2.24(\mathrm{~m}, 3 \mathrm{H}), 2.18-2.05(\mathrm{~m}, 1 \mathrm{H}), 1.92-1.80(\mathrm{~m}, 1 \mathrm{H}), 1.78-1.65(\mathrm{~m}, 1 \mathrm{H}), 1.62-1.50(\mathrm{~m}, 2 \mathrm{H}), 1.48-$ $1.35(\mathrm{~m}, 1 \mathrm{H})$.

${ }^{13}$ C NMR (125 MHz, $\left.\mathrm{CD}_{2} \mathrm{Cl}_{2}\right): \delta$ 198.7, 80.7, 51.2, 34.0, 30.6, 30.4, 23.2.

HRMS $(\mathrm{m} / \mathrm{z})$ calcd for $\mathrm{C}_{7} \mathrm{H}_{12} \mathrm{O}_{2} \mathrm{SNa}[\mathrm{M}]^{+\mathrm{Na}}: 183.0456$, found: 183.0448 .

$[\alpha]_{\mathbf{D}}^{25}:+81.1^{\circ}\left(c=0.375, \mathrm{CHCl}_{3}\right)$.

The enantiomeric ratio was determined by HPLC analysis using Daicel Chiralcel AD-3 column: $n \mathrm{Hept}: \mathrm{PrOH}=90: 10$, flow rate $1.0 \mathrm{~mL} / \mathrm{min}, \tau_{1}=5.1 \mathrm{~min}, \tau_{2}=5.4 \mathrm{~min}$.

\subsubsection{Organocascade products}

\section{$5 \mathrm{a}-(1 R, 2 R)-2$-mercaptocyclohexyl benzoate -}

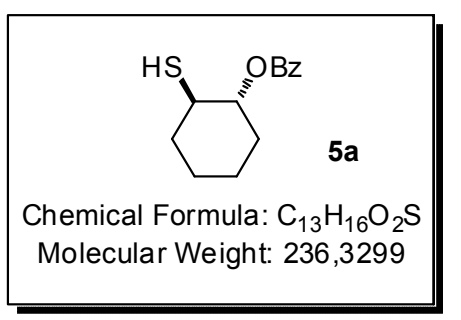

The reaction was performed at $-78^{\circ} \mathrm{C}$ and the product was isolated as a colorless liquid in $86 \%$ yield and e.r. $=98: 2$.

${ }^{1} \mathbf{H}$ NMR $\left(300 \mathrm{MHz}, \mathrm{CDCl}_{3}\right): \delta$ 8.12-8.02 (m, 2H), 7.62-7.53 (m, 1H), 7.52-7.40 (m, 2H), 4.95-4.80 (cm, 1H), 3.15-2.95 (m, 1H), 2.30-2.10 (m, 2H), 1.92-1.68 (m, 2H), $1.73(\mathrm{~d}, J=1.73 \mathrm{~Hz}, 1 \mathrm{H}), 1.62-$ $1.20(\mathrm{~m}, 4 \mathrm{H})$.

${ }^{13}$ C NMR (75 MHz, $\left.\mathrm{CDCl}_{3}\right): \delta 166.1,133.1,130.7,129.9,128.6,79.0,42.4,35.1,31.6,25.6,24.2$.

HRMS $(m / z)$ calcd for $\mathrm{C}_{13} \mathrm{H}_{16} \mathrm{O}_{2} \mathrm{SNa}[\mathrm{M}]^{+\mathrm{Na}}: 259.0763$, found: 259.0762 .

$[\alpha]_{\mathbf{D}}^{25}:-89.7^{\circ}\left(c=0.925, \mathrm{CHCl}_{3}\right)$.

The enantiomeric ratio was determined by HPLC analysis using Daicel Chiralcel AD-3 column: $n$ Hept: $i \mathrm{PrOH}=98: 2$, flow rate $1.0 \mathrm{~mL} / \mathrm{min}, \tau_{1}=4.5 \mathrm{~min}, \tau_{2}=5.0 \mathrm{~min}$. 


\section{$5 \mathrm{~b}-(1 R, 6 R)-6$-mercaptocyclohex-3-en-1-yl benzoate -}

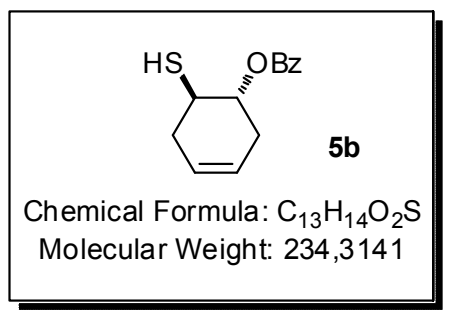

The reaction was performed at $-40^{\circ} \mathrm{C}$ and the product was isolated as a colorless liquid in $98 \%$ yield and e.r. $=96: 4$.

${ }^{1} \mathbf{H}$ NMR $\left(300 \mathrm{MHz}, \mathrm{CD}_{2} \mathrm{Cl}_{2}\right): \delta$ 8.00-7.92 (m, 2H), 7.55-7.45 (m, 1H), 7.42-7.32 (m, 2H), $5.58(\mathrm{~cm}$, 2H), 5.10-5.00 (m, 1H), 3.32-3.18 (m, 1H), 2.78-2.58 (m, 2H), 2.25-2.05 (m, 2H), $1.77(\mathrm{~d}, J=7.1 \mathrm{~Hz}$, $1 \mathrm{H})$.

${ }^{13}$ C NMR (75 MHz, $\left.\mathrm{CD}_{2} \mathrm{Cl}_{2}\right): \delta$ 166.1, 133.4, 130.8, 129.9, 128.8, 124.9, 124.3, 74.6, 37.6, 33.7, 30.0. HRMS $(m / z)$ calcd for $\mathrm{C}_{13} \mathrm{H}_{14} \mathrm{O}_{2} \mathrm{SNa}[\mathrm{M}]^{+\mathrm{Na}}: 257.0607$, found: 257.0607 .

$[\alpha]_{\mathbf{D}}{ }^{25}:-145.6^{\circ}\left(c=1.100, \mathrm{CHCl}_{3}\right)$.

The enantiomeric ratio was determined by HPLC analysis using Daicel Chiralcel AD-3 column: $n$ Hept: $i \mathrm{PrOH}=98: 2$, flow rate $1.0 \mathrm{~mL} / \mathrm{min}, \tau_{1}=4.2 \mathrm{~min}, \tau_{2}=4.6 \mathrm{~min}$.

\section{$5 c-(2 R, 3 R)-3-m e r c a p t o-1,2,3,4-t e t r a h y d r o n a p h t h a l e n-2-y l$ benzoate -}

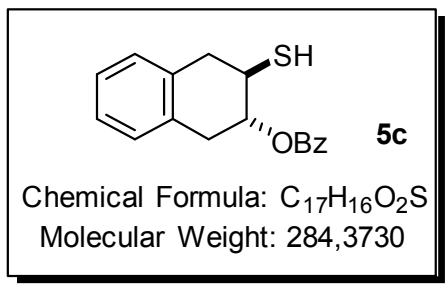

The reaction was performed at $-40^{\circ} \mathrm{C}$ and the product was isolated as a colorless liquid in 97\% yield and e.r. $=95: 5$.

${ }^{1}$ H NMR $\left(300 \mathrm{MHz}, \mathrm{CDCl}_{3}\right): \delta$ 8.10-8.02 (m, 2H), 7.65-7.54 (m, 1H), 7.50-7.40 (m, 2H), 7.25-7.08 (m, 4H), $5.37(\mathrm{~cm}, 1 \mathrm{H}), 3.62-3.40(\mathrm{~m}, 3 \mathrm{H}), 3.08-2.92(\mathrm{~m}, 2 \mathrm{H}), 1.84(\mathrm{~d}, J=7.0 \mathrm{~Hz}, 1 \mathrm{H})$

${ }^{13}$ C NMR (75 MHz, $\left.\mathrm{CDCl}_{3}\right): \delta 166.1,133.4,133.3,133.0,130.4,129.9,129.3,128.9,128.7,126.9$, 126.7, 74.9, 38.0, 36.7, 33.1.

HRMS $(m / z)$ calcd for $\mathrm{C}_{17} \mathrm{H}_{16} \mathrm{O}_{2} \mathrm{SNa}[\mathrm{M}]^{+\mathrm{Na}}$ : 307.0763 , found: 307.0763 .

$[\alpha]_{\mathbf{D}}{ }^{25}:-132.2^{\circ}\left(c=1.325, \mathrm{CHCl}_{3}\right)$.

The enantiomeric ratio was determined by HPLC analysis using Daicel Chiralcel AD-3 column: $n \mathrm{Hept}: i \mathrm{PrOH}=98: 2$, flow rate $1.0 \mathrm{~mL} / \mathrm{min}, \tau_{1}=8.0 \mathrm{~min}, \tau_{2}=9.3 \mathrm{~min}$. 
$5 \mathrm{e}-(1 R, 2 R)-2$-mercaptocycloheptyl benzoate -

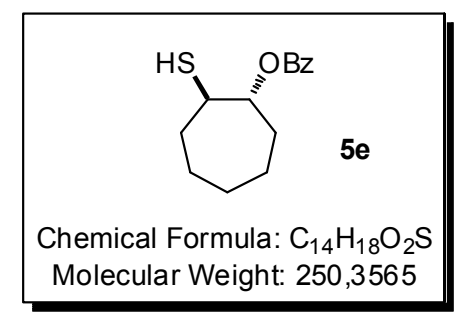

The reaction was performed at $-40^{\circ} \mathrm{C}$ and the product was isolated as a colorless liquid in $80 \%$ yield and e.r. $=96: 4$.

${ }^{1} \mathbf{H}$ NMR $\left(300 \mathrm{MHz}, \mathrm{CDCl}_{3}\right): \delta$ 8.15-8.00 (m, 2H), 7.64-7.54 (m, 1H), 7.52-7.40 (m, 2H), 5.09 (dt, $J$ $=3.1 \mathrm{~Hz} J=7.8 \mathrm{~Hz}, 1 \mathrm{H}), 3.38-3.25(\mathrm{~m}, 1 \mathrm{H}), 2.20-2.00(\mathrm{~m}, 2 \mathrm{H}), 1.95-1.50(\mathrm{~m}, 9 \mathrm{H})$.

${ }^{13} \mathbf{C}$ NMR $\left(75 \mathrm{MHz}, \mathrm{CDCl}_{3}\right): \delta 165.9,133.2,130.7,129.8,128.6,81.9,44.6,33.8,31.7,28.0,25.3$, 22.4 .

HRMS $(m / z)$ calcd for $\mathrm{C}_{14} \mathrm{H}_{18} \mathrm{O}_{2} \mathrm{SNa}[\mathrm{M}]^{+\mathrm{Na}}: 273.0919$, found: 273.0920 .

$[\alpha]_{\mathbf{D}}^{25}:-76.2^{\circ}\left(c=0.900, \mathrm{CHCl}_{3}\right)$.

The enantiomeric ratio was determined by HPLC analysis using Daicel Chiralcel AD-3 column: $n$ Hept: $i \mathrm{PrOH}=98: 2$, flow rate $1.0 \mathrm{~mL} / \mathrm{min}, \tau_{1}=5.0 \mathrm{~min}, \tau_{2}=5.5 \mathrm{~min}$.

$5 \mathrm{~h}-(2 R, 3 R)-3-$ mercaptobutan-2-yl benzoate -

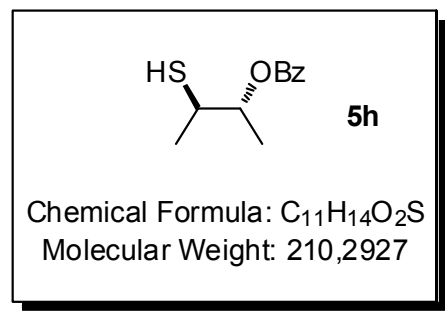

The reaction was performed at $-40^{\circ} \mathrm{C}$ and the product was isolated as a colorless liquid in $81 \%$ yield and e.r. $=97: 3$.

${ }^{1} \mathbf{H}$ NMR $\left(300 \mathrm{MHz}, \mathrm{CDCl}_{3}\right): \delta$ 8.13-8.05 (m, 2H), 7.65-7.55 (m, 1H), 7.50-7.42 (m, 2H), 5.19 (dq, $J$ $=4.6 \mathrm{~Hz} J=6.2 \mathrm{~Hz}, 1 \mathrm{H}), 3.19(\mathrm{dQ}, J=4.6 \mathrm{~Hz} J=7.2 \mathrm{~Hz}, 1 \mathrm{H}), 1.65$ (d, $J=7.2 \mathrm{~Hz} 1 \mathrm{H}), 1.42(\mathrm{~cm}$, $6 \mathrm{H})$.

${ }^{13}$ C NMR (125 MHz, $\left.\mathrm{CDCl}_{3}\right): \delta 166.0,133.2,130.6,129.8,128.6,75.2,39.3,20.9,16.8$.

HRMS $(m / z)$ calcd for $\mathrm{C}_{11} \mathrm{H}_{14} \mathrm{O}_{2} \mathrm{SNa}[\mathrm{M}]^{+\mathrm{Na}}: 233.0607$, found: 233.0609 .

$[\alpha]_{\mathbf{D}}^{25}:-39.4^{\circ}\left(c=0.350, \mathrm{CHCl}_{3}\right)$.

The enantiomeric ratio was determined by HPLC analysis using Daicel Chiralcel OJ-H column: $n$ Hept $: i \mathrm{PrOH}=99.5: 0.5$, flow rate $0.5 \mathrm{~mL} / \mathrm{min}, \tau_{1}=14.6 \mathrm{~min}, \tau_{2}=15.5 \mathrm{~min}$. 


\section{$5 \mathrm{i}-(4 R, 5 R)-5$-mercaptooctan-4-yl benzoate -}

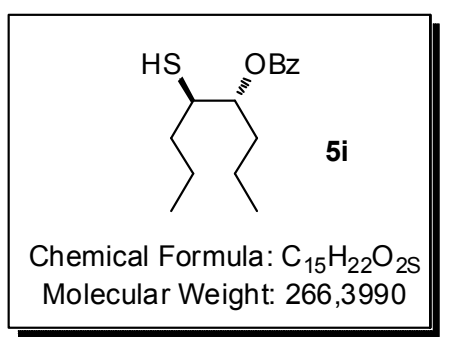

The reaction was performed at $-40^{\circ} \mathrm{C}$ and the product was isolated as a colorless liquid in $82 \%$ yield and e.r. $=94: 6$.

${ }^{1} \mathbf{H}$ NMR $\left(300 \mathrm{MHz}, \mathrm{CDCl}_{3}\right): \delta$ 8.15-8.03 (m, 2H), 7.65-7.55 (m, 1H), 7.52-7.43 (m, 2H), $5.28(\mathrm{~cm}$, 1H), 3.08-2.95 (m, 1H), 1.95-1.30 (m, 9H), 0.96 (t, $J=7.4 \mathrm{~Hz}, 3 \mathrm{H}), 0.91(\mathrm{t}, J=7.4 \mathrm{~Hz}, 3 \mathrm{H})$.

${ }^{13} \mathbf{C}$ NMR $\left(75 \mathrm{MHz}, \mathrm{CDCl}_{3}\right): \delta 166.3,133.2,130.5,129.9,128.6,77.0,44.0,37.8,33.9,20.8,19.1$, 14.1, 13.9.

HRMS $(m / z)$ calcd for $\mathrm{C}_{15} \mathrm{H}_{22} \mathrm{O}_{2} \mathrm{SNa}[\mathrm{M}]^{+\mathrm{Na}}: 289.1233$, found: 289.1233 .

$[\alpha]_{\mathbf{D}}^{25}:+15.4^{\circ}\left(c=0.350, \mathrm{CHCl}_{3}\right)$.

The enantiomeric ratio was determined by HPLC analysis using Daicel Chiralcel OJ-3R column: $\mathrm{CH}_{3} \mathrm{CN}: \mathrm{H}_{2} \mathrm{O}=50: 50$, flow rate $1.0 \mathrm{~mL} / \mathrm{min}, \tau_{1}=17.6 \mathrm{~min}, \tau_{2}=18.6 \mathrm{~min}$.

\section{$5 \mathrm{j}-(3 R, 4 R)-4-m e r c a p t o h e x a n-3-y l$ benzoate -}

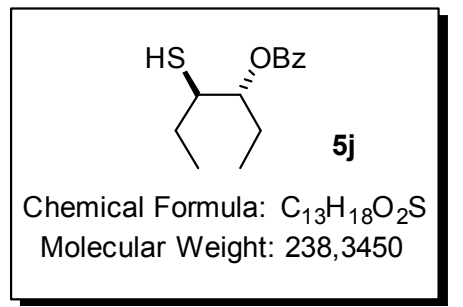

The reaction was performed at $-40^{\circ} \mathrm{C}$ and the product was isolated as a colorless liquid in $74 \%$ yield and e.r. $=93.5: 6.5$.

${ }^{1} \mathbf{H}$ NMR $\left(300 \mathrm{MHz}, \mathrm{CD}_{2} \mathrm{Cl}_{2}\right): \delta$ 8.10-7.90 (m, 2H), 7.55-7.45 (m, 1H), 7.42-7.32 (m, 2H), $5.09(\mathrm{~cm}$, $1 \mathrm{H}), 2.84(\mathrm{~cm}, 1 \mathrm{H}), 1.88-1.64(\mathrm{~m}, 3 \mathrm{H}), 1.54-1.35(\mathrm{~m}, 2 \mathrm{H}), 0.97$ (t, $J=7.4 \mathrm{~Hz}, 3 \mathrm{H}), 0.86$ (t, $J=7.4 \mathrm{~Hz}$, $3 \mathrm{H})$.

${ }^{13}$ C NMR (75 MHz, $\left.\mathrm{CD}_{2} \mathrm{Cl}_{2}\right): \delta 166.3,133.3,130.9,129.9,128.8,78.4,46.0,29.1,25.1,12.3,10.2$.

HRMS $(\mathrm{m} / \mathrm{z})$ calcd for $\mathrm{C}_{13} \mathrm{H}_{18} \mathrm{O}_{2} \mathrm{SNa}[\mathrm{M}]^{+\mathrm{Na}}: 261.0920$, found: 261.0921 .

$[\alpha]_{\mathbf{D}}^{25}:+12.0^{\circ}\left(c=0.750, \mathrm{CHCl}_{3}\right)$.

The enantiomeric ratio was determined by HPLC analysis using Daicel Chiralcel OJ-H column: $n \mathrm{Hept}: \mathrm{PrOH}=99.5: 0.5$, flow rate $0.5 \mathrm{~mL} / \mathrm{min}, \tau_{1}=11.6 \mathrm{~min}, \tau_{2}=12.3 \mathrm{~min}$. 
$5 \mathrm{k}-(1 R, 2 R)-2$-mercapto-1,2-diphenylethyl benzoate -

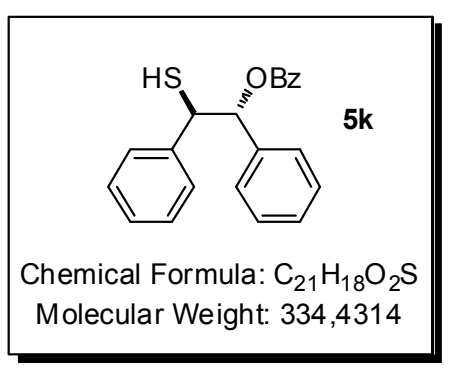

The reaction was performed at $-10^{\circ} \mathrm{C}$ and the product was isolated as a white solid in $99 \%$ yield and e.r. $=87: 13$. Single recrystallization in pentane afforded the product in $70 \%$ yield and e.r. $=98.5: 1.5$.

${ }^{1} \mathbf{H}$ NMR $\left(300 \mathrm{MHz}, \mathrm{CDCl}_{3}\right): \delta$ 8.25-8.08 (m, 2H), 7.68-7.56 (m, 1H), 7.55-7.43 (m, 2H), 7.38-7.10 (m, 10H), $6.27(\mathrm{~d}, J=8.4 \mathrm{~Hz}, 1 \mathrm{H}), 4.59(\mathrm{dd}, J=8.4 \mathrm{~Hz} J=5.0 \mathrm{~Hz}, 1 \mathrm{H}), 2.31(\mathrm{~d}, J=5.0 \mathrm{~Hz} 1 \mathrm{H})$.

${ }^{13} \mathbf{C}$ NMR $\left(75 \mathrm{MHz}, \mathrm{CDCl}_{3}\right): \delta 165.5,139.4,138.0,133.4,130.2,130.0,128.7,128.7,128.5,128.4$, $128.3,128.0,127.4,80.7,49.7$.

HRMS $(m / z)$ calcd for $\mathrm{C}_{21} \mathrm{H}_{18} \mathrm{O}_{2} \mathrm{SNa}[\mathrm{M}]^{+\mathrm{Na}}: 357.0920$, found: 357.0921 .

$[\alpha]_{\mathbf{D}}{ }^{25}:-2.7^{\circ}\left(c=0.450, \mathrm{CHCl}_{3}\right)$.

The enantiomeric ratio was determined by HPLC analysis using Daicel Chiralcel AD-3 column: $n \mathrm{Hept}: i \mathrm{PrOH}=85: 5$, flow rate $1.0 \mathrm{~mL} / \mathrm{min}, \tau_{1}=6.0 \mathrm{~min}, \tau_{2}=9.0 \mathrm{~min}$.

\section{$5 \mathrm{I}-(1 R, 2 R)-2$-mercaptocyclohexyl acetate-}

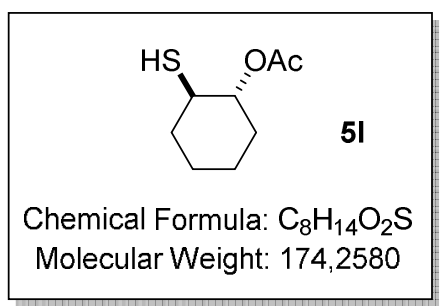

The reaction was performed at $-40^{\circ} \mathrm{C}$ and the product was isolated as a colorless liquid in $86 \%$ yield and e.r. $=95: 5$.

${ }^{1} \mathbf{H}$ NMR $\left(500 \mathrm{MHz}, \mathrm{CD}_{2} \mathrm{Cl}_{2}\right): \delta 4.47(\mathrm{td}, J=10.0 \mathrm{~Hz}, J=4.4 \mathrm{~Hz}, 1 \mathrm{H}), 2.80-2.70(\mathrm{~m}, 1 \mathrm{H}), 2.06-1.90$ (m, 2H), 1.97 (s, 3H), 1.70-1.58 (m, 2H), 1.62 (d, $J=6.1 \mathrm{~Hz}, 1 \mathrm{H}), 1.43-1.15(\mathrm{~m}, 4 \mathrm{H})$.

${ }^{13}$ C NMR (125 MHz, $\left.\mathrm{CD}_{2} \mathrm{Cl}_{2}\right): \delta 170.5,78.5,42.6,35.5,31.9,25.9,24.5,21.3$.

HRMS $(\mathrm{m} / \mathrm{z})$ calcd for $\mathrm{C}_{8} \mathrm{H}_{14} \mathrm{O}_{2} \mathrm{SNa}[\mathrm{M}]^{+\mathrm{Na}}: 197.0607$, found: 197.0608 .

$[\alpha]_{\mathbf{D}}^{25}:-53.8^{\circ}\left(c=0.520, \mathrm{CHCl}_{3}\right)$.

The enantiomeric ratio was determined by GC analysis using BGB-177/BG-15 column $30 \mathrm{~m}\left(80{ }^{\circ} \mathrm{C}\right.$, $0.5^{\circ} \mathrm{C} / \mathrm{min}$ until $120^{\circ} \mathrm{C}, 10{ }^{\circ} \mathrm{C} / \mathrm{min}$ until $220^{\circ} \mathrm{C}, 3 \mathrm{~min}$ at $220^{\circ} \mathrm{C}, 0.4$ bar $\left.\mathrm{H}_{2}\right), \tau_{1}=64.0 \mathrm{~min}, \tau_{2}=65.6$ $\min$. 


\section{$5 n-(1 R, 2 R)-2$-mercapto-1,2-diphenylethyl acetate-}

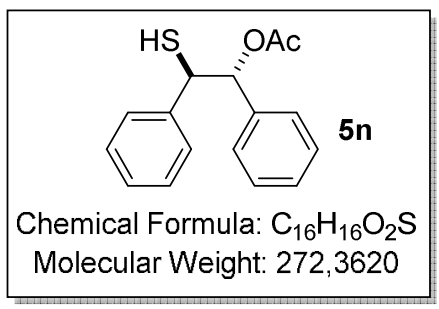

The reaction was performed at $10{ }^{\circ} \mathrm{C}$ and the product was isolated as a colorless liquid in $63 \%$ yield and e.r. $=86: 14$.

${ }^{1}$ H NMR $\left(500 \mathrm{MHz}, \mathrm{CD}_{2} \mathrm{Cl}_{2}\right): \delta$ 7.20-7-00 (m, 10H), $5.87(\mathrm{~d}, J=8.90 \mathrm{~Hz}, 1 \mathrm{H}), 4.31(\mathrm{dd}, J=8.9 \mathrm{~Hz}$, $J=5.1 \mathrm{~Hz}, 1 \mathrm{H}), 2.27(\mathrm{~d}, J=5.1 \mathrm{~Hz}, 1 \mathrm{H}), 2.03(\mathrm{~s}, 3 \mathrm{H})$.

${ }^{13}$ C NMR $\left(125 \mathrm{MHz}, \mathrm{CD}_{2} \mathrm{Cl}_{2}\right): \delta 170.1,139.7,138.4,128.8,128.5,128.4,128.4,128.1,127.5,80.3$, 49.4, 21.2.

HRMS $(m / z)$ calcd for $\mathrm{C}_{16} \mathrm{H}_{16} \mathrm{O}_{2} \mathrm{SNa}[\mathrm{M}]^{+\mathrm{Na}}: 295.0769$, found: 295.0775 .

$[\alpha]_{\mathbf{D}}^{25}:-59.5^{\circ}\left(c=0.740, \mathrm{CHCl}_{3}\right)$.

The enantiomeric ratio was determined by HPLC analysis using Daicel Chiralcel AD-3 column: $n \mathrm{Hept}: i \mathrm{PrOH}=90: 10$, flow rate $1.0 \mathrm{~mL} / \mathrm{min}, \tau_{1}=5.6 \mathrm{~min}, \tau_{2}=6.0 \mathrm{~min}$. 


\section{One-pot synthesis of 1,2-thioalcohols}
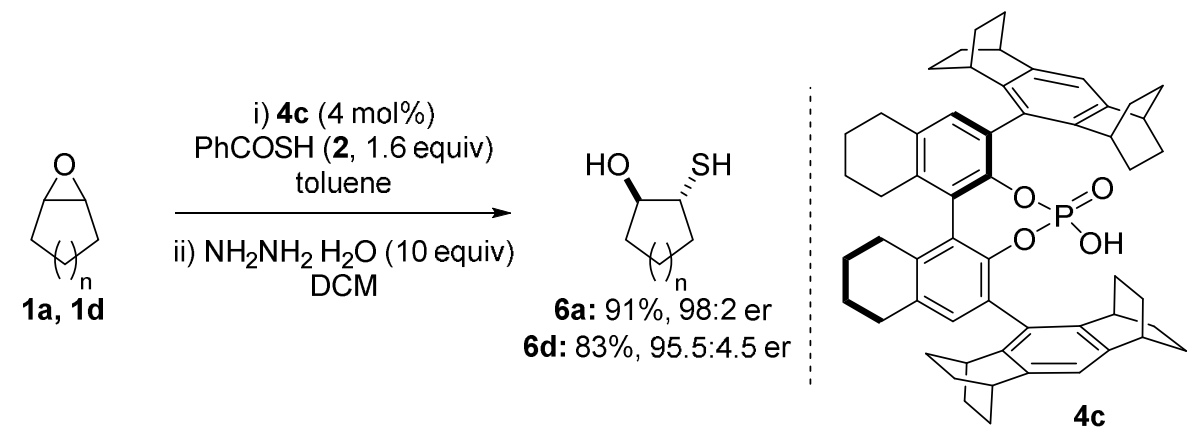

In a screw-cap vial, thiobenzoic acid 2 ( $0.32 \mathrm{mmol}, 1.6$ equiv.) and catalyst $4 \mathbf{c}$ ( $4 \mathrm{~mol} \%)$ were dissolved in $0.8 \mathrm{~mL}$ of toluene and cooled to the appropriate reaction temperature $\left(1 \mathrm{a}:-78^{\circ} \mathrm{C}\right.$, 1d: $\left.-40{ }^{\circ} \mathrm{C}\right)$. Then a pre-cooled solution of epoxide $1(0.2 \mathrm{mmol})$ in $0.8 \mathrm{~mL}$ of toluene was added and the reaction was stirred for 2-3 days. Next, the reaction temperature was slowly raised to r.t. and a solution of hydrazine hydrate (100 mg, $2 \mathrm{mmol}, 10$ equiv.) in $0.5 \mathrm{~mL}$ of dichloromethane was added dropwise and the mixture was stirred for $2 \mathrm{~h}$ at room temperature. The mixture was diluted with dichloromethane and the 1,2-thioalcohols were directly purified by column chromatography on silica gel (eluent: dichloromethane).

Upon comparison with previous literature reports, the absolute configuration of 2mercaptocyclohexanol 6a was found to be $R, R .^{3}$

\section{6a - $(1 R, 2 R)-2$ mercaptocyclohexanol -}

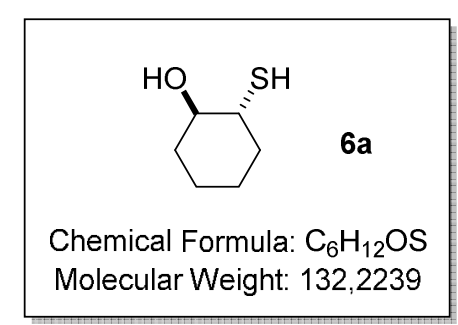

Isolated in $91 \%$ yield $(24 \mathrm{mg}, 0.18 \mathrm{mmol})$ and $98: 2 \mathrm{er}$.

${ }^{1} \mathbf{H}$ NMR $\left(500 \mathrm{MHz}, \mathrm{CD}_{2} \mathrm{Cl}_{2}\right): \delta 3.08(\mathrm{mc}, 1 \mathrm{H}), 2.55(\mathrm{~d}, J=2.70 \mathrm{~Hz}$, $1 \mathrm{H}), 2.47-2.35(\mathrm{~m}, 1 \mathrm{H}), 2.10-1.87(\mathrm{~m}, 2 \mathrm{H}), 1.73-1.52(\mathrm{~m}, 2 \mathrm{H}), 1.42$ $(\mathrm{d}, J=8.7 \mathrm{~Hz}, 1 \mathrm{H}), 1.34-1.05(\mathrm{~m}, 4 \mathrm{H})$.

${ }^{13}$ C NMR (125 MHz, $\left.\mathrm{CD}_{2} \mathrm{Cl}_{2}\right): \delta 76.9,48.0,36.8,34.5,26.9,25.1$.

HRMS $(\mathrm{m} / \mathrm{z})$ calcd for $\mathrm{C}_{6} \mathrm{H}_{12} \mathrm{OS}[\mathrm{M}]: 132.0609$, found: 132.0609 .

$[\alpha]_{\mathbf{D}}{ }^{25}:-73.3^{\circ}\left(c=0.410, \mathrm{CHCl}_{3}\right)$.

The enantiomeric ratio was determined by GC analysis using BGB-178-BG-15 column $30 \mathrm{~m}$ (115 min at $75{ }^{\circ} \mathrm{C}, 10{ }^{\circ} \mathrm{C} / \mathrm{min}$ until $220^{\circ} \mathrm{C}, 4 \mathrm{~min}$ at $220^{\circ} \mathrm{C}, 0.5$ bar $\left.\mathrm{H}_{2}\right), \tau_{1}=99.9 \mathrm{~min}, \tau_{2}=103.2 \mathrm{~min}$.

\footnotetext{
${ }^{3}$ Wang, Z.; Law, W. K.; Sun, J. Org. Lett. 2013, 15, 5964.
} 


\section{6d - (1R,2R)-2mercaptocyclopentanol -}

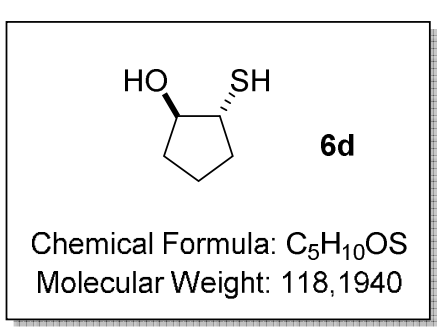

Isolated in $83 \%$ yield $(19.5 \mathrm{mg}, 0.165 \mathrm{mmol})$ and $95.5: 4.5 \mathrm{er}$.

${ }^{1} \mathbf{H}$ NMR $\left(500 \mathrm{MHz}, \mathrm{CD}_{2} \mathrm{Cl}_{2}\right): \delta 3.80(\mathrm{mc}, 1 \mathrm{H}), 2.83(\mathrm{Q}, J=7.1 \mathrm{~Hz}$, $1 \mathrm{H}), 2.18-2.05(\mathrm{~m}, 1 \mathrm{H}), 2.02-1.92(\mathrm{~m}, 1 \mathrm{H}), 1.86(\mathrm{~d}, J=3.8 \mathrm{~Hz}, 1 \mathrm{H})$, $1.75-1.60(\mathrm{~m}, 2 \mathrm{H}), 1.52(\mathrm{~d}, J=7.1 \mathrm{~Hz}, 1 \mathrm{H}), 1.45-1.38(\mathrm{~m}, 2 \mathrm{H})$.

${ }^{13}$ C NMR (125 MHz, $\left.\mathrm{CD}_{2} \mathrm{Cl}_{2}\right): \delta 82.1,46.4,33.9,32.6,21.1$.

HRMS $(m / z)$ calcd for $\mathrm{C}_{5} \mathrm{H}_{9} \mathrm{OS}[\mathrm{M}]^{-\mathrm{H}}: 117.0380$, found: 117.0379 .

$[\alpha]_{\mathbf{D}}^{25}:-45.5^{\circ}\left(c=0.440, \mathrm{CHCl}_{3}\right)$.

The enantiomeric ratio was determined by GC analysis using BGB-177/BGB-15 column $25 \mathrm{~m}$ (40 min at $100{ }^{\circ} \mathrm{C}, 10{ }^{\circ} \mathrm{C} / \mathrm{min}$ until $220^{\circ} \mathrm{C}, 3 \mathrm{~min}$ at $220^{\circ} \mathrm{C}, 0.4$ bar $\left.\mathrm{H}_{2}\right), \tau_{1}=29.9 \mathrm{~min}, \tau_{2}=30.7 \mathrm{~min}$. 


\section{2 mmol-scale preparation of compound 5a}

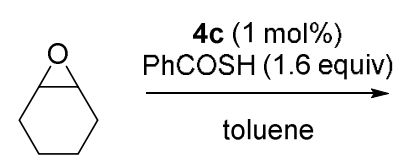

$1 \mathrm{a}$

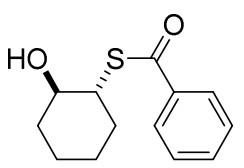

$3 \mathbf{a}$

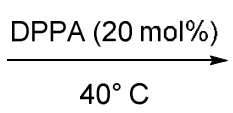

$196 \mathrm{mg}, 2.0 \mathrm{mmol}$

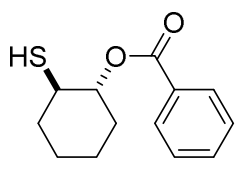

$5 \mathbf{a}$

$448 \mathrm{mg}, 1.9 \mathrm{mmol}$ $95 \%$ yield, $97.5: 2.5 \mathrm{er}$

In a $50 \mathrm{ml}$ round bottom flask, cyclohexene oxide 1a (196 mg, $2.0 \mathrm{mmol})$ was dissolved in toluene $(8 \mathrm{ml})$ and the solution was cooled at $-78^{\circ} \mathrm{C}$. Then a pre-cooled solution of catalyst 4c (16.5 mg, $0.02 \mathrm{mmol}, 1 \mathrm{~mol} \%)$ and thiobenzoic acid (441 mg, $3.2 \mathrm{mmol}, 1.6$ equiv) in toluene $(8 \mathrm{ml})$ was added. The reaction was stirred at $-78^{\circ} \mathrm{C}$ for 3 days and then slowly warmed to room temperature. Next, a solution of diphenyl phosphoric acid (DPPA, 50 $\mathrm{mg}, 0.2 \mathrm{mmol}, 10 \mathrm{~mol} \%)$ in toluene $(0.2 \mathrm{ml})$ was added and the reaction was heated at $40^{\circ} \mathrm{C}$ for two additional days. Dichloromethane was added and the organic solution was washed with an aqueous $\mathrm{NaOH}$ solution (2 M), dried over $\mathrm{MgSO}_{4}$, and concentrated in vacuo. After fast purification by column chromatography on silica gel (eluent: mixtures hexanes/MTBE) thiol 5a was obtained in 95\% yield (448 mg, $1.9 \mathrm{mmol}, 97.5: 2.5 \mathrm{er}$ ).

NOTE: The addition of DPPA for the acyl-transfer step is due to the lower loading of catalyst $\mathbf{4 c}$ in the transformation. 


\section{Copies of NMR spectra}

\section{Epoxides - Starting materials}
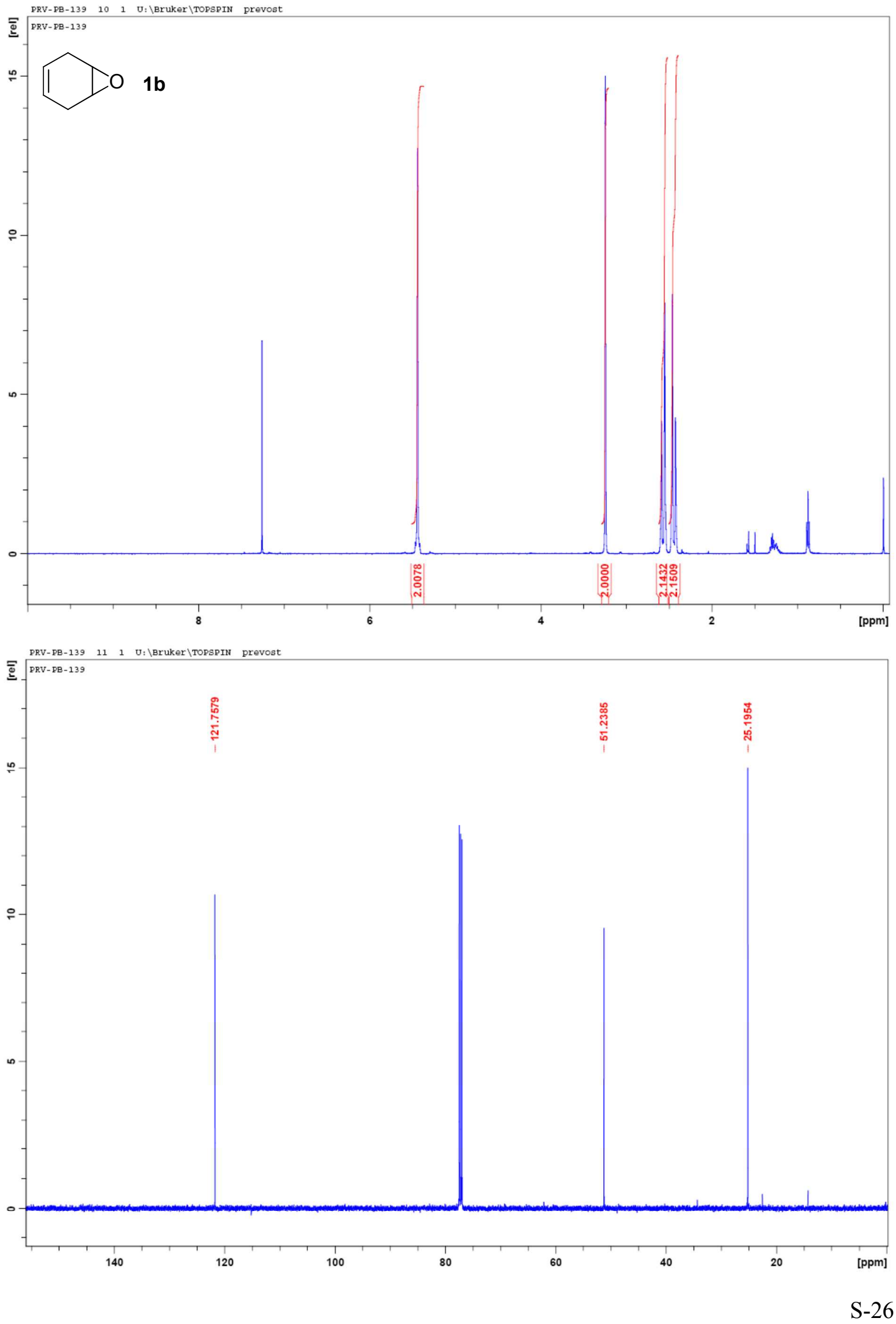

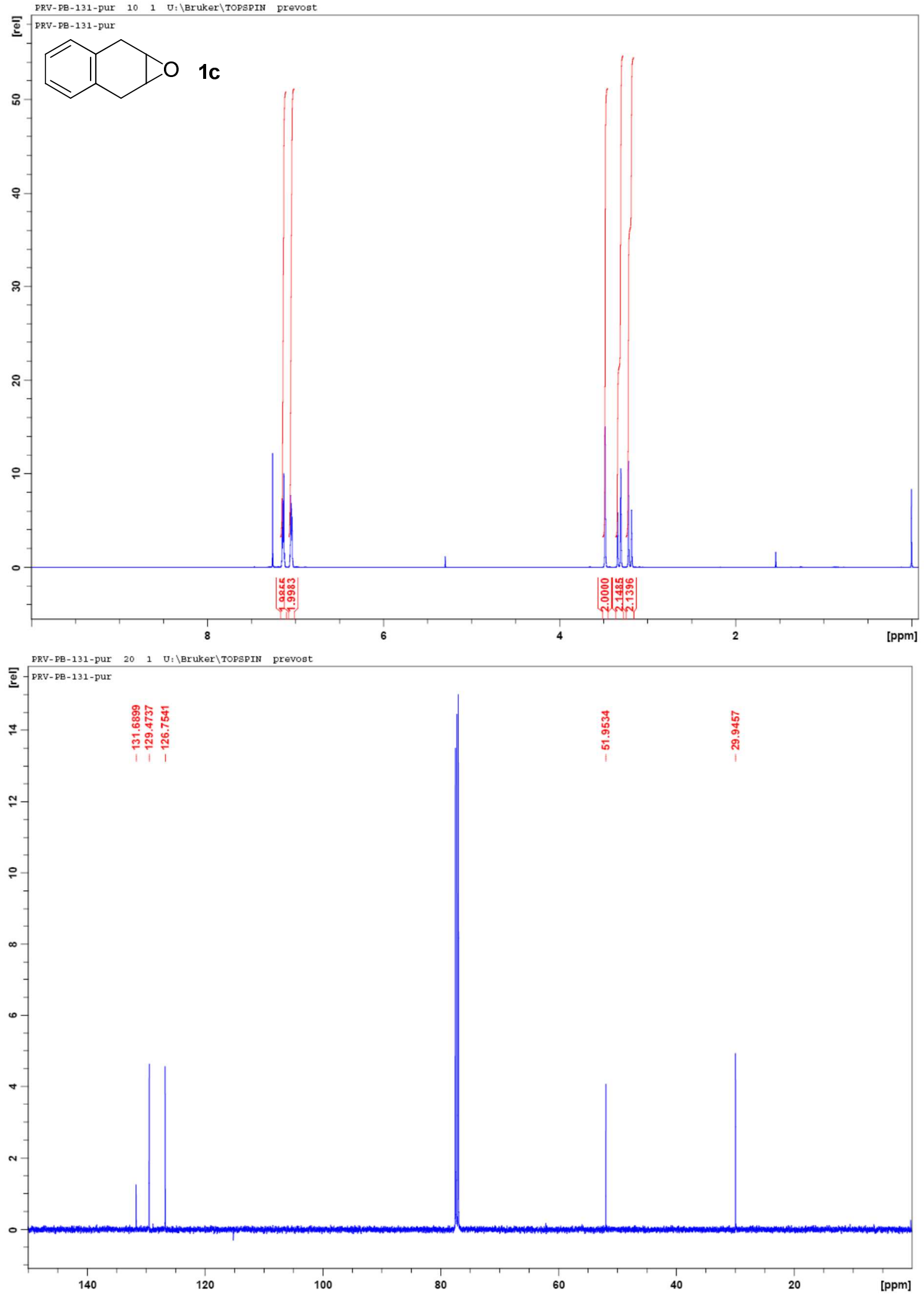

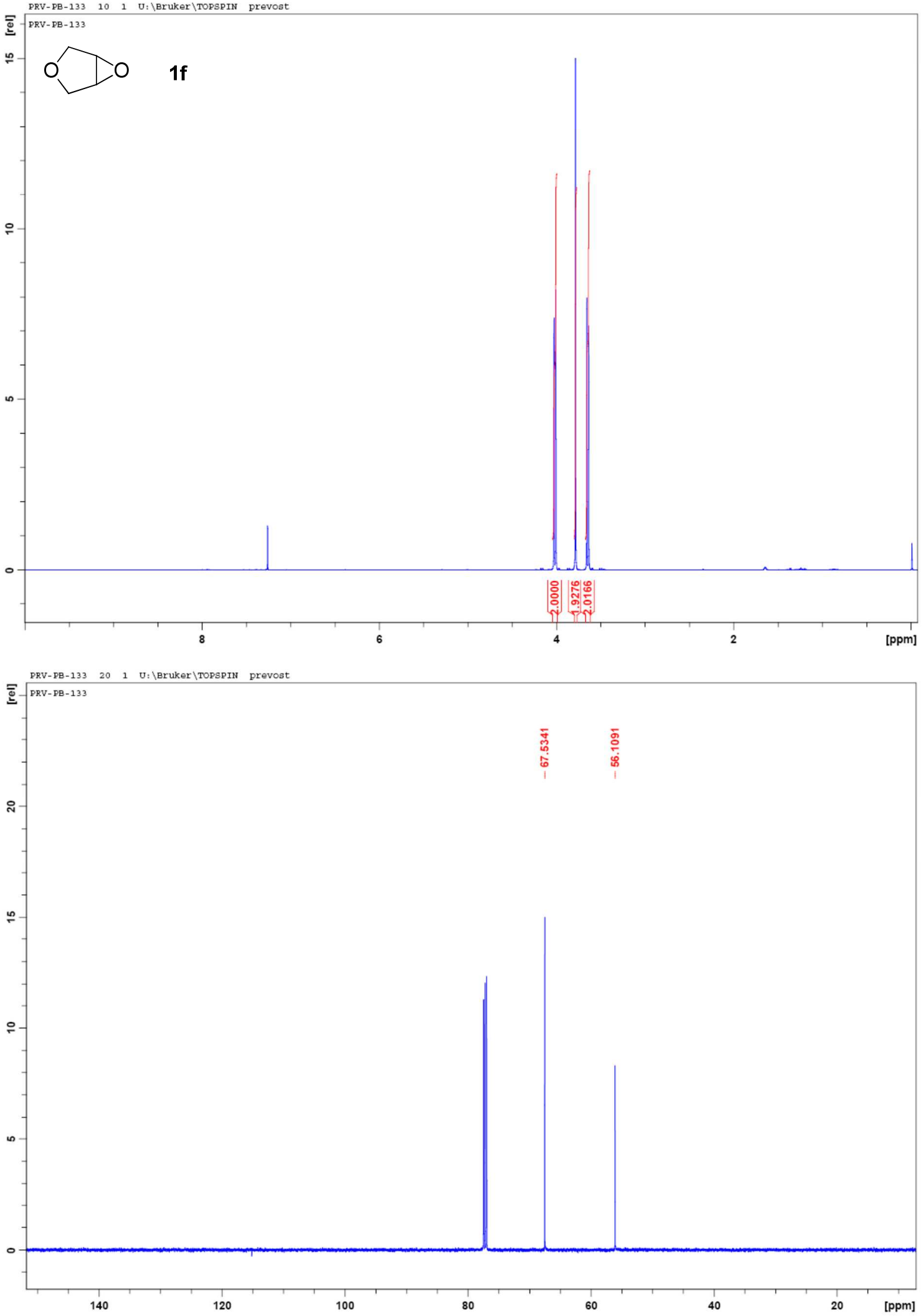

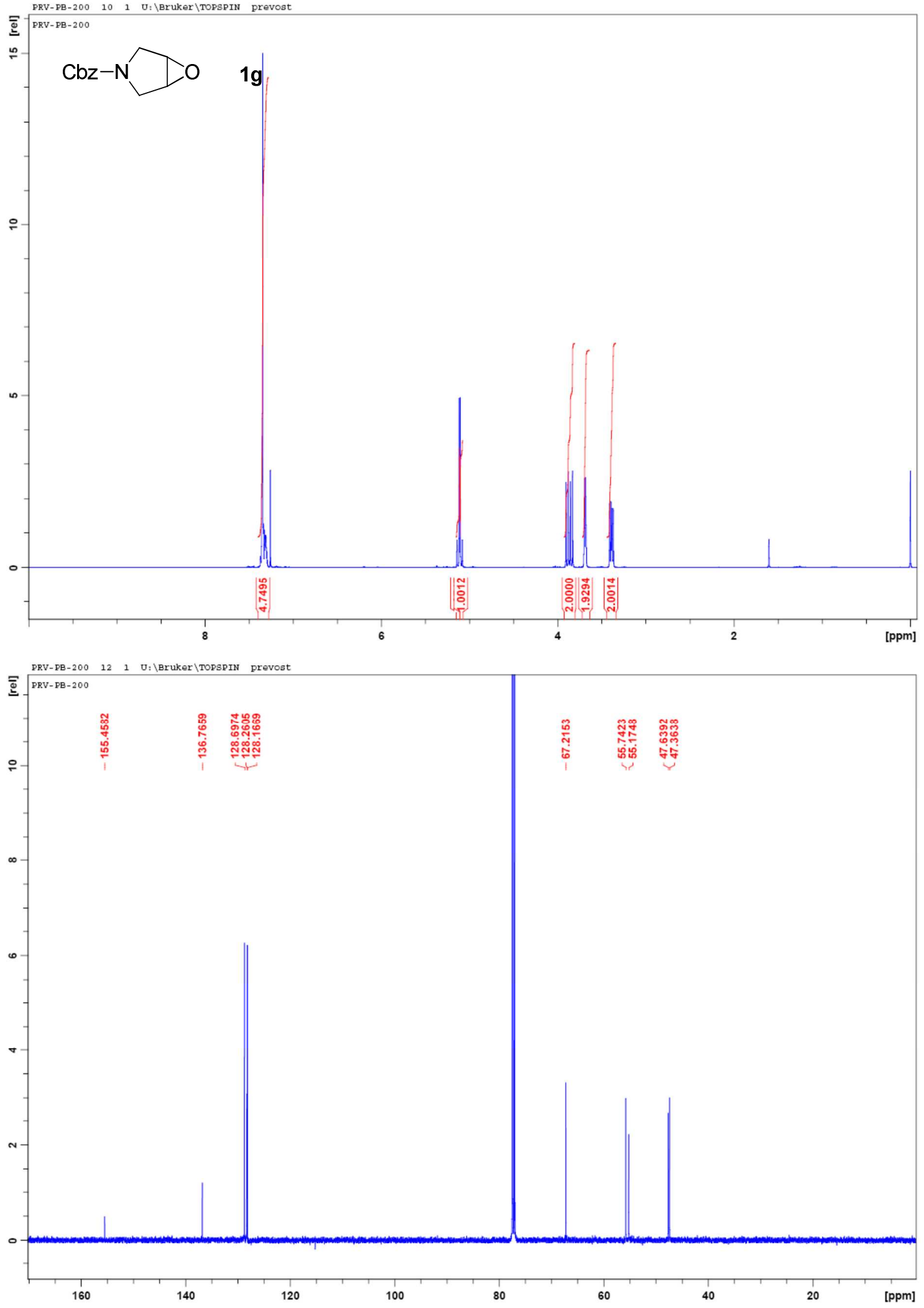

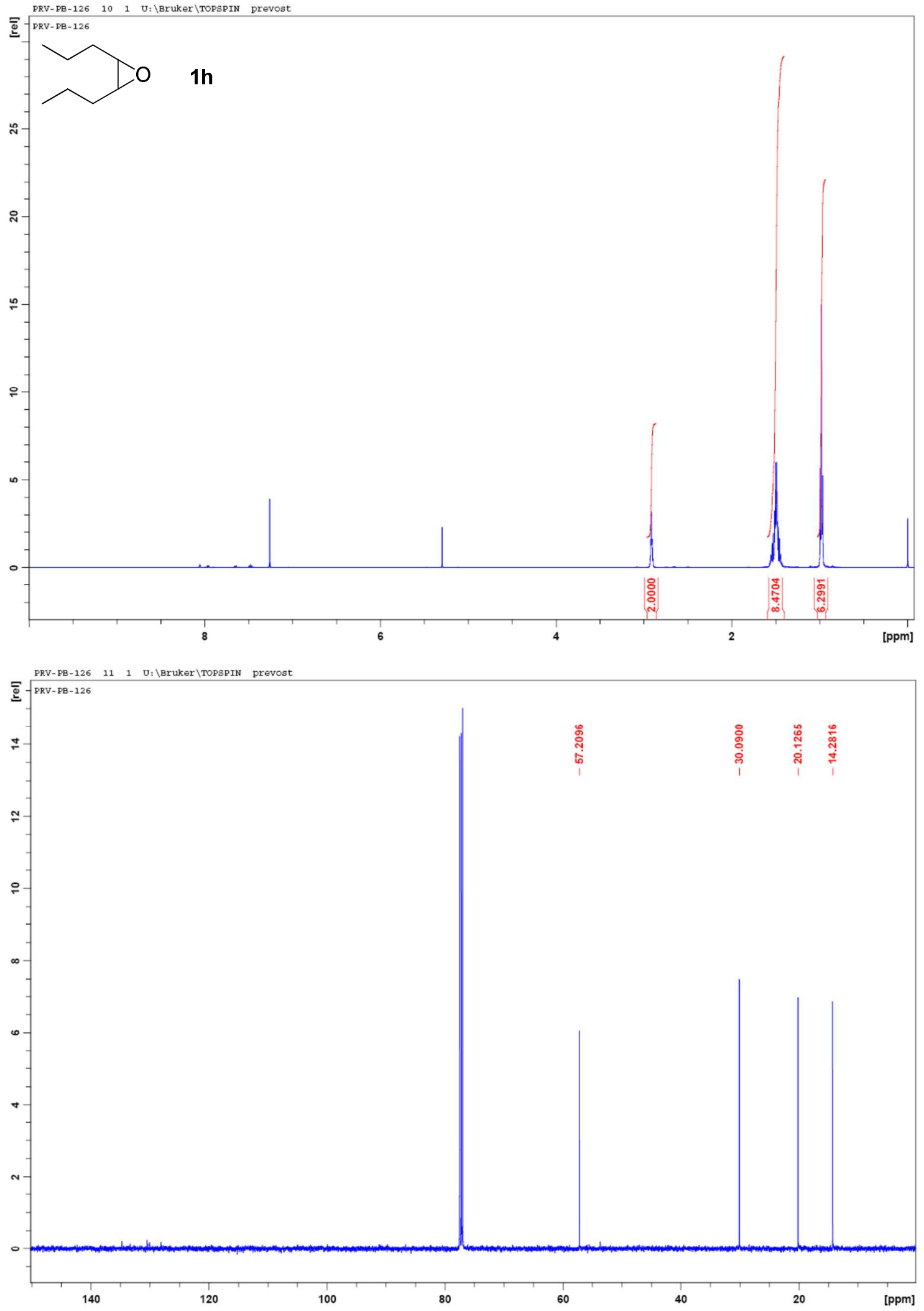
PRV-PB-140 $10 \quad 1 \quad U: \backslash$ Bruker $\backslash$ TOPSPIN prevost
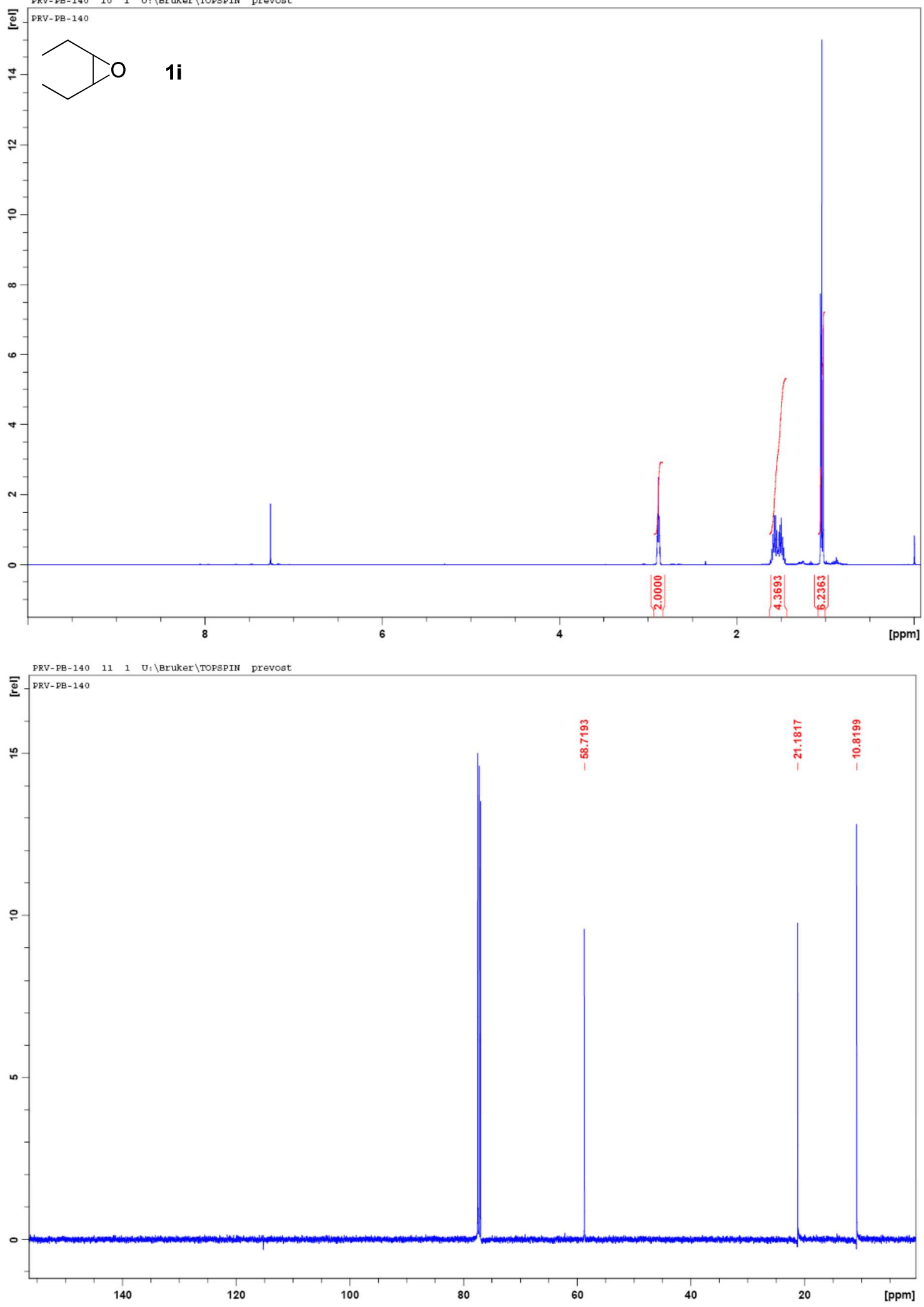


\section{Products}
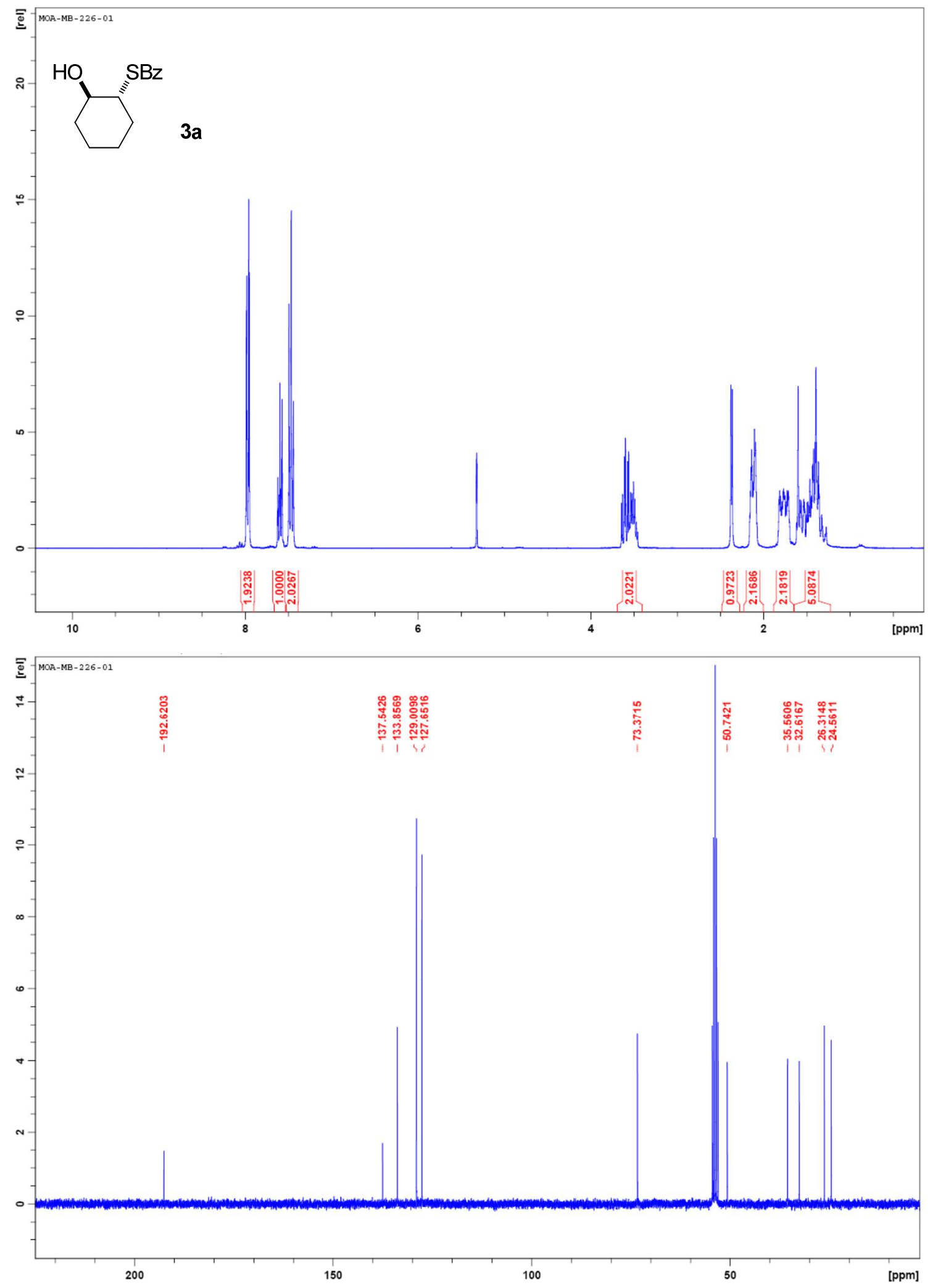

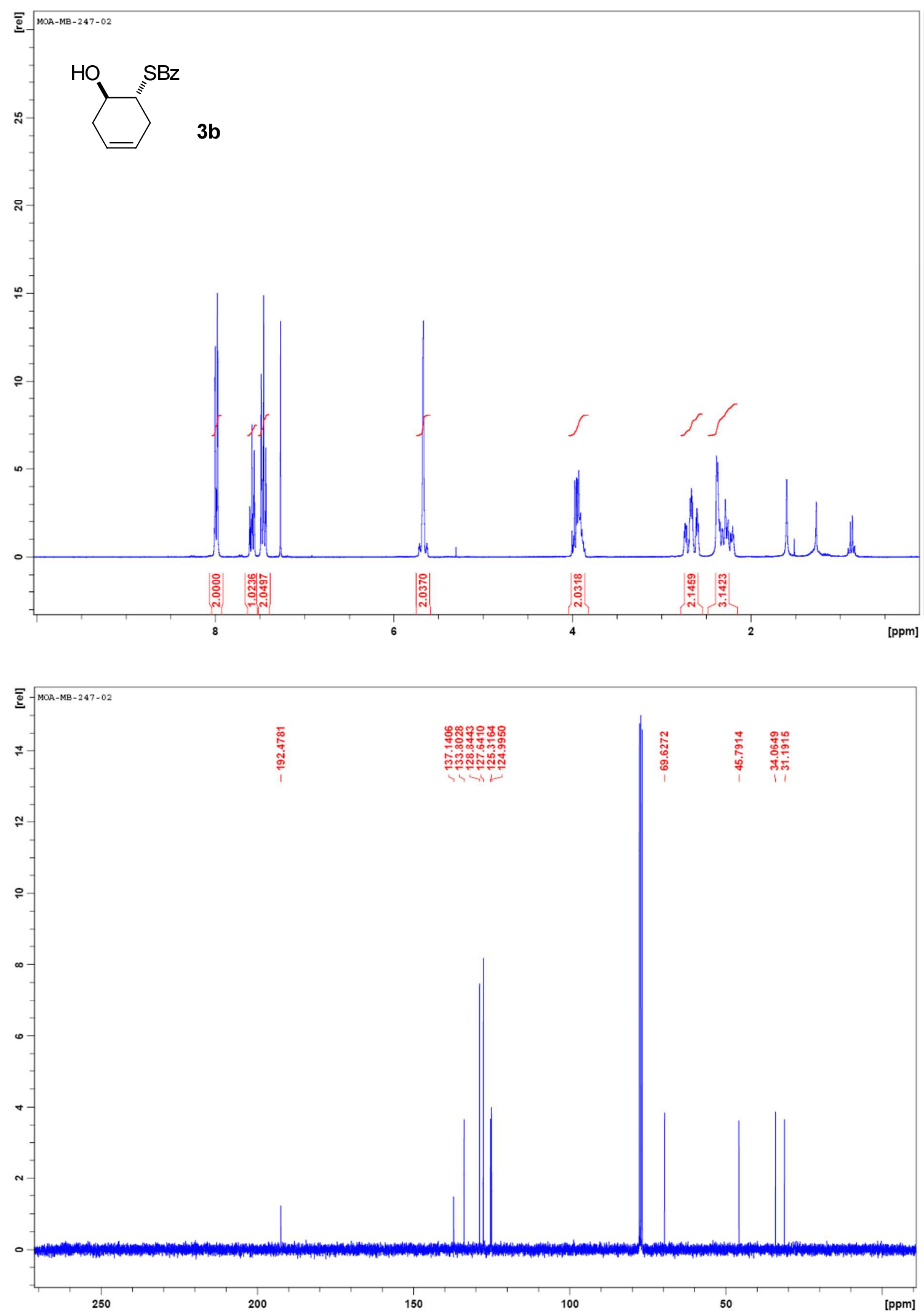

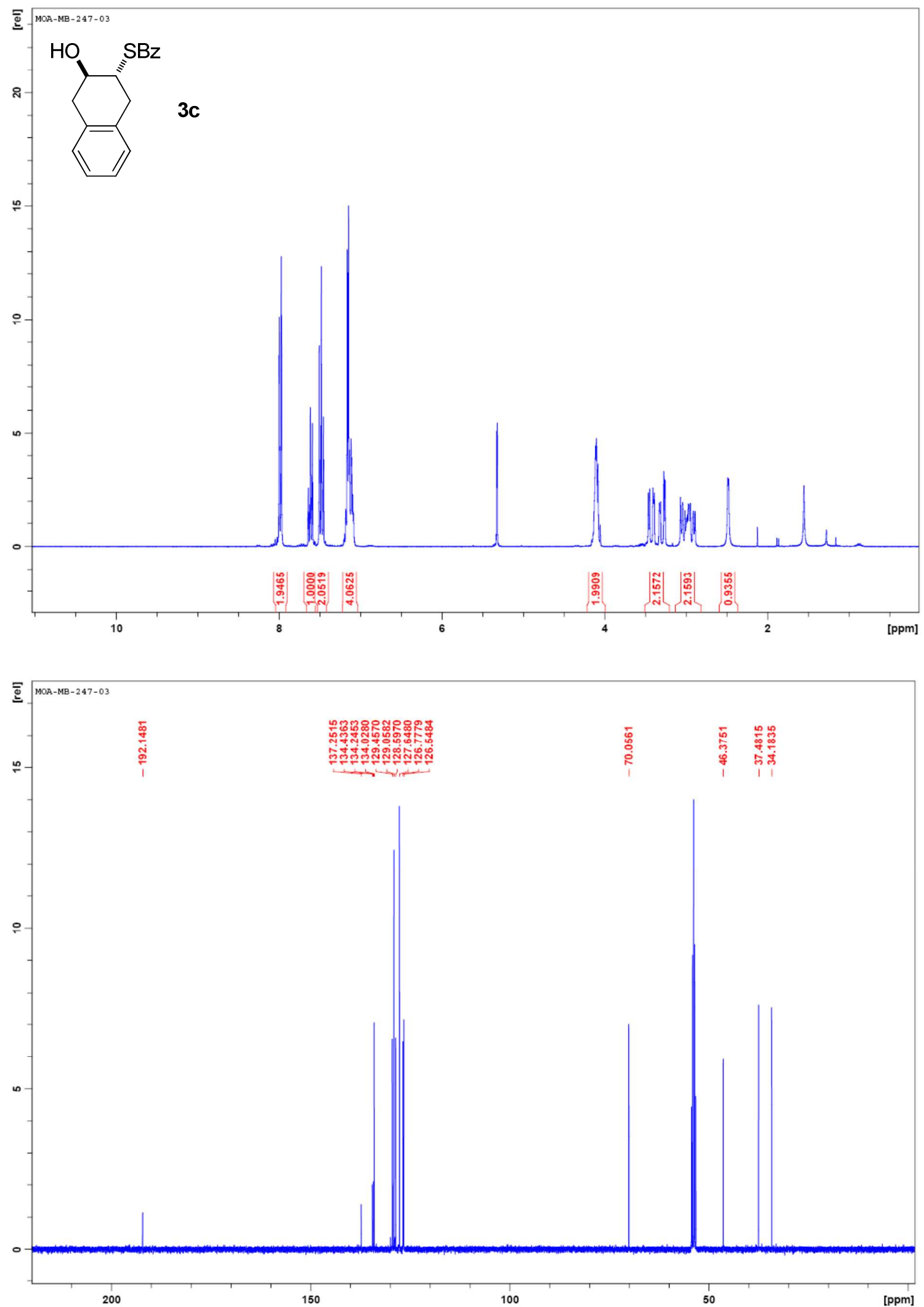

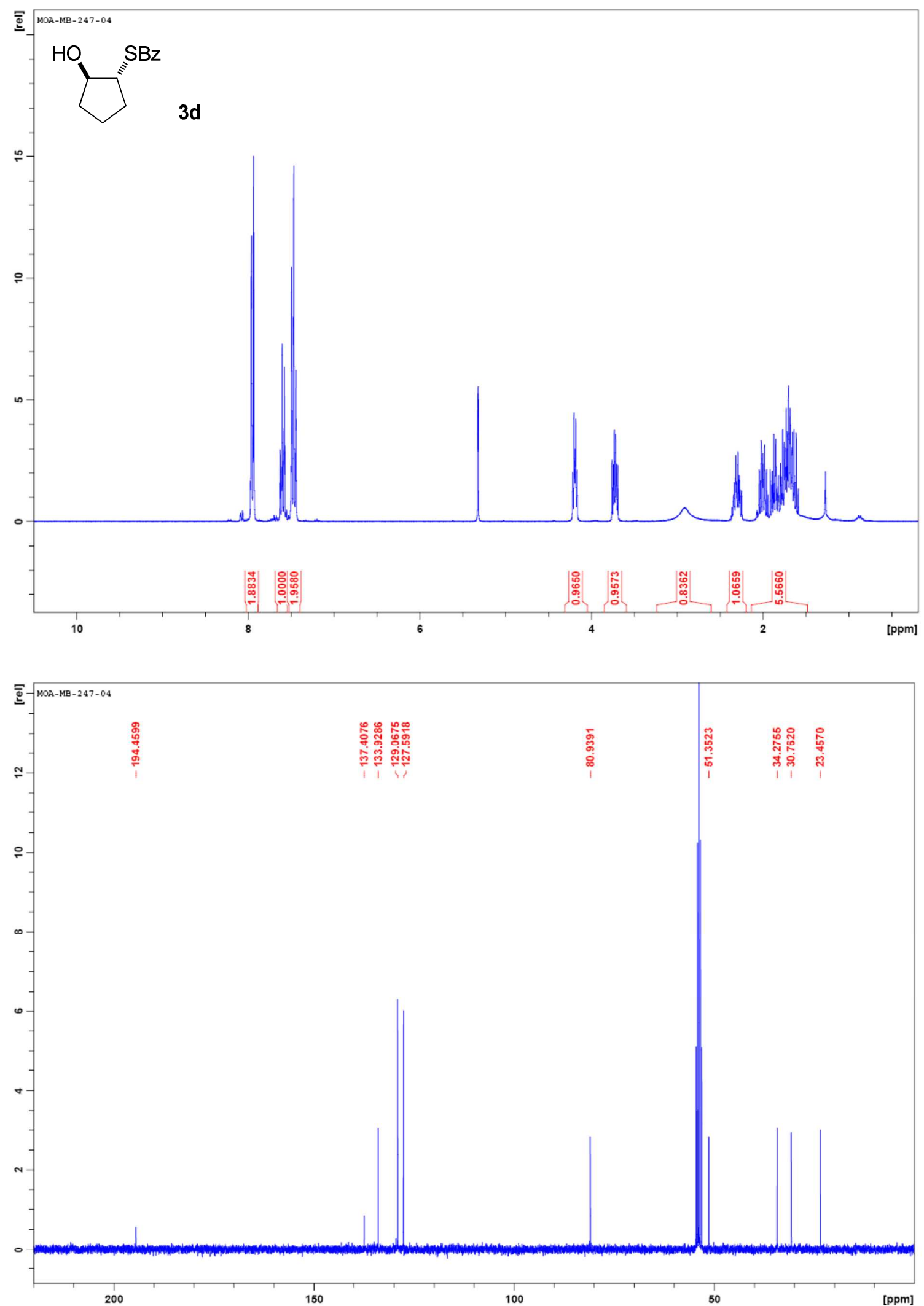

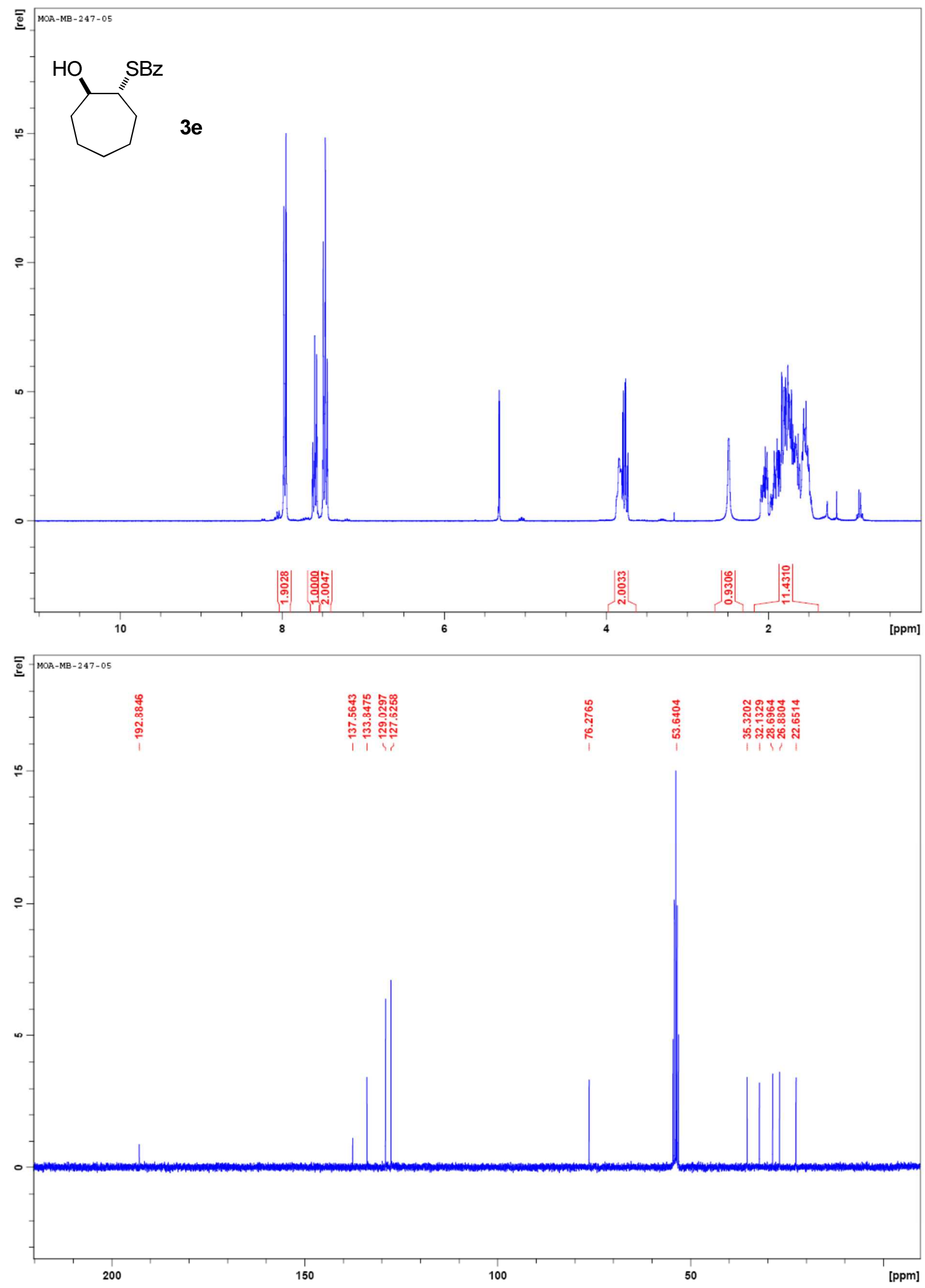

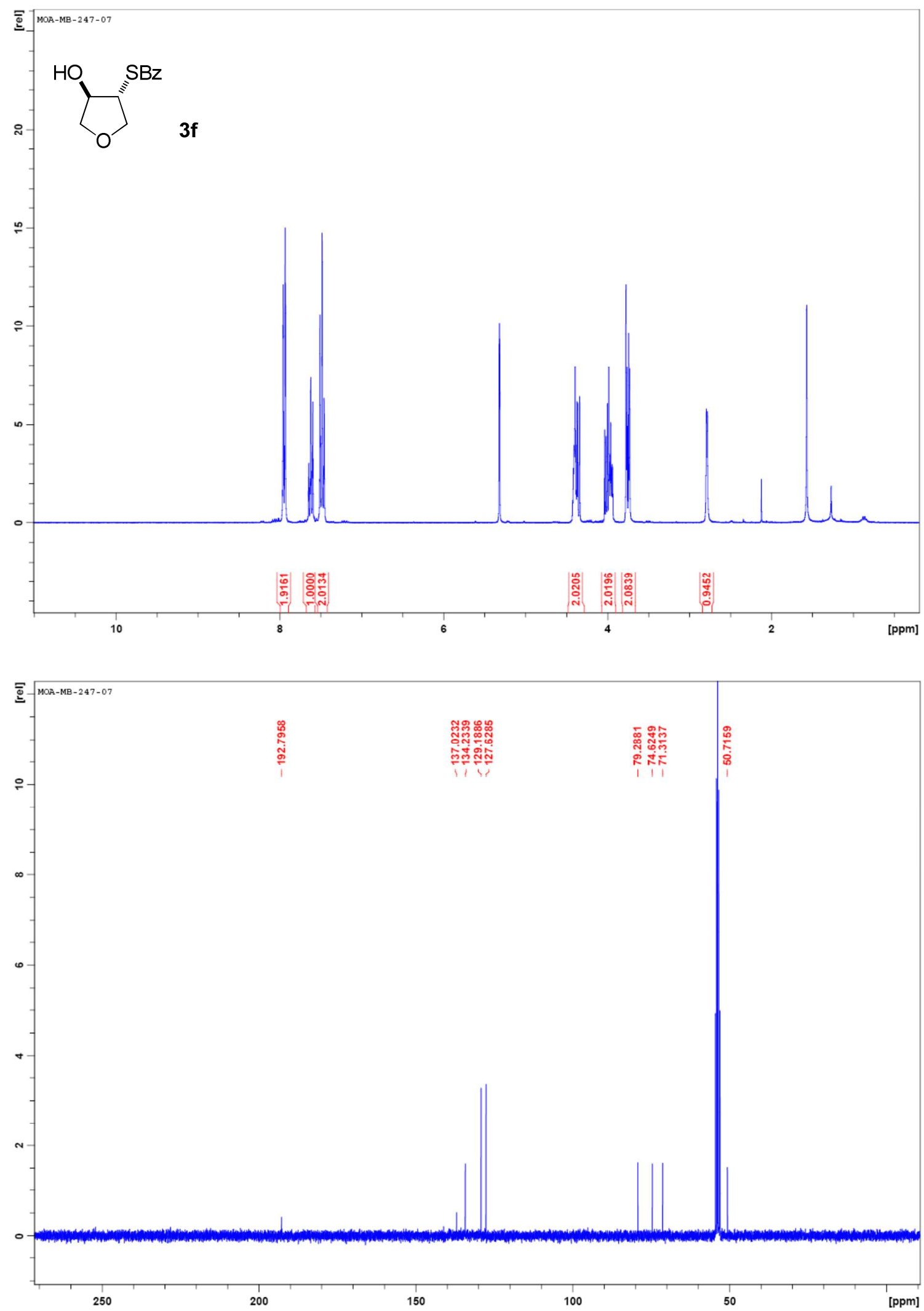

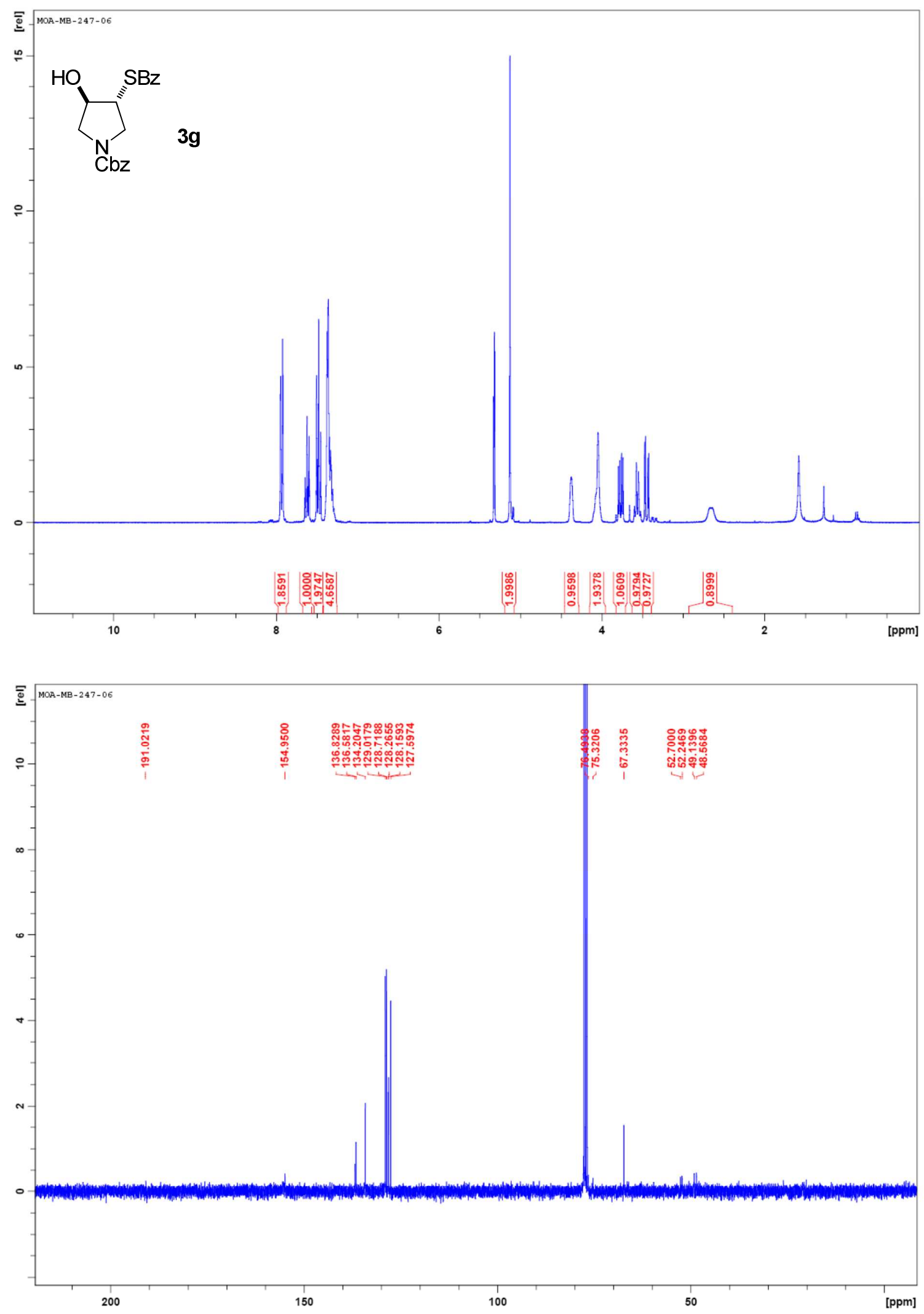

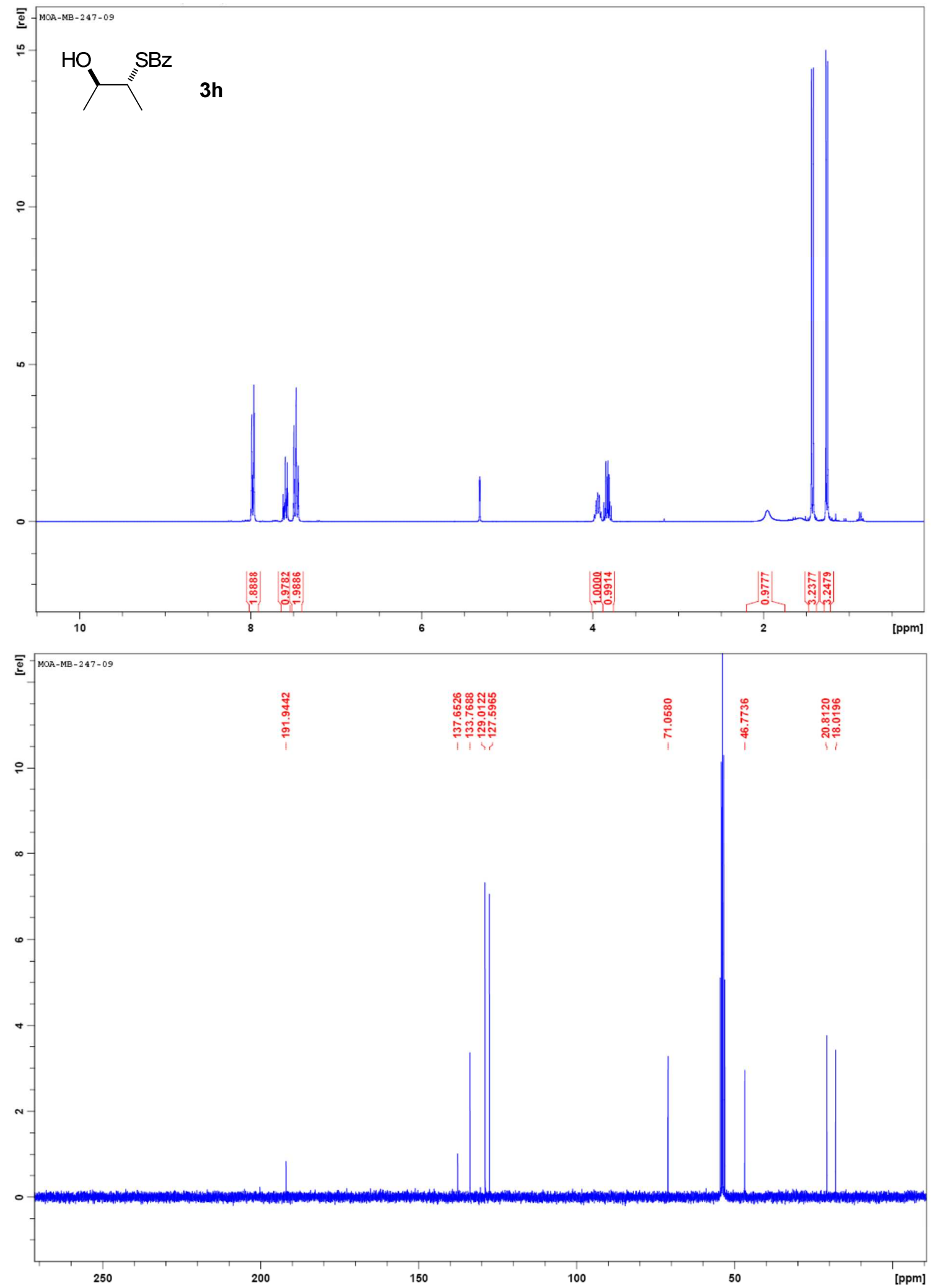

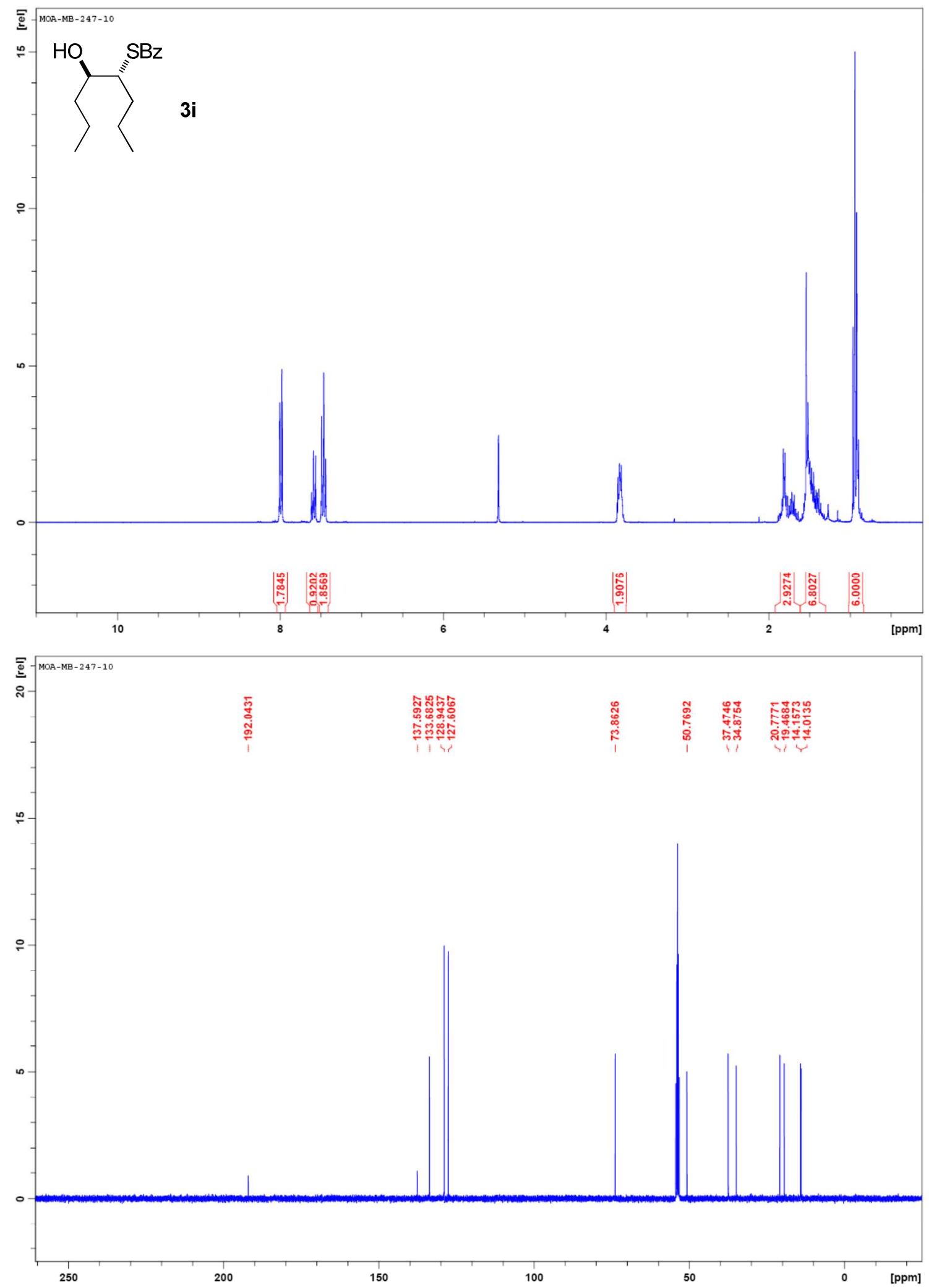

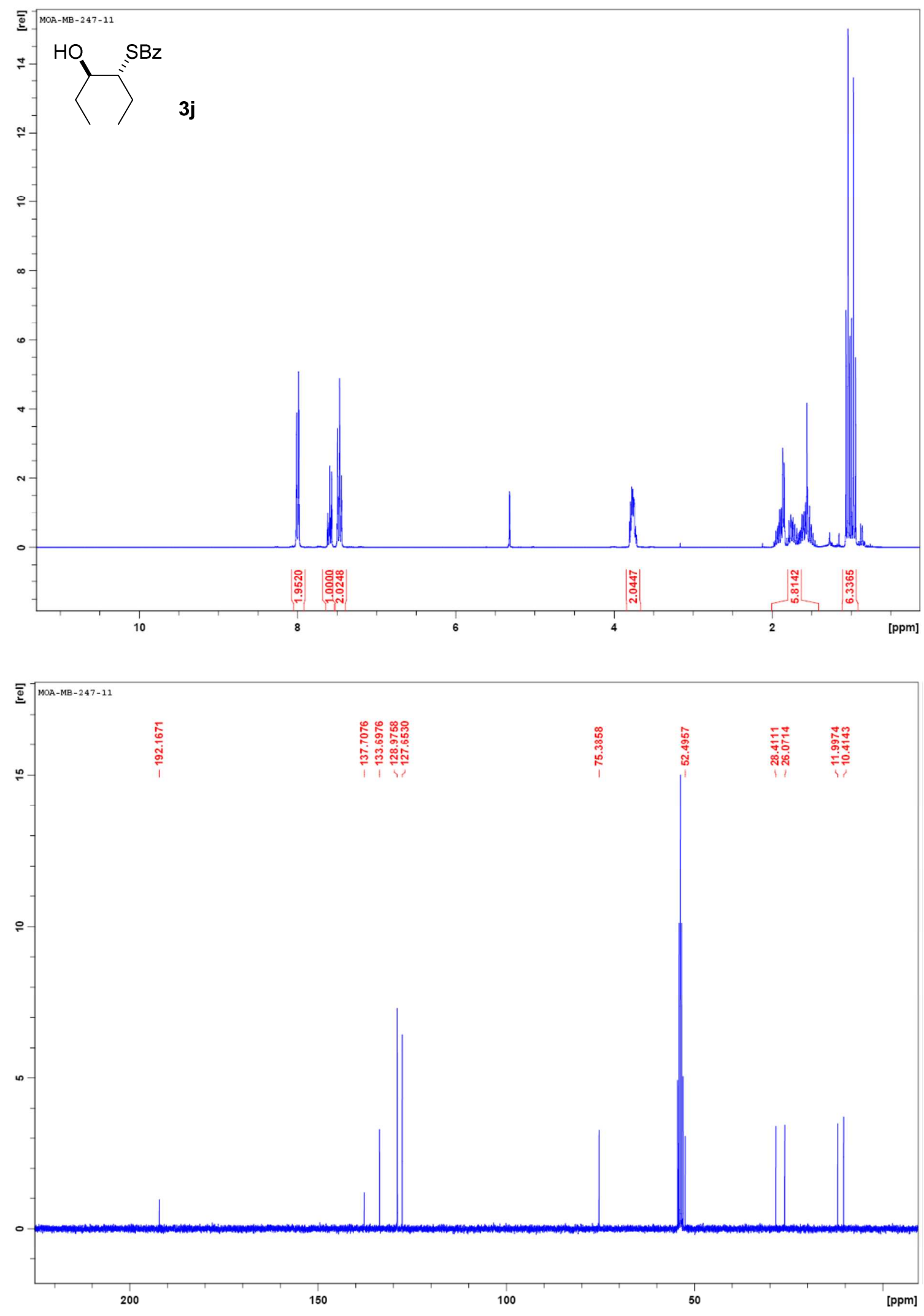

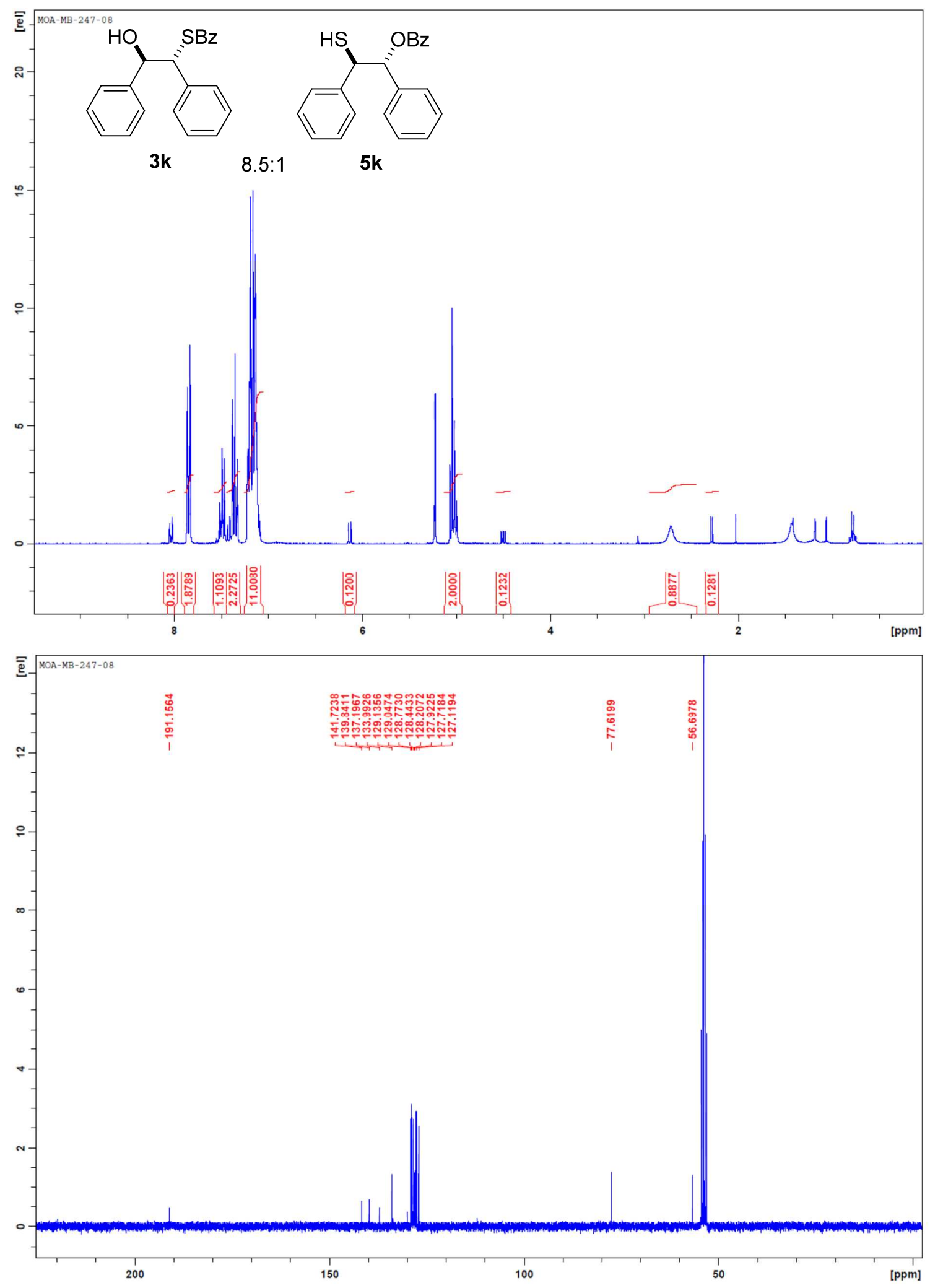

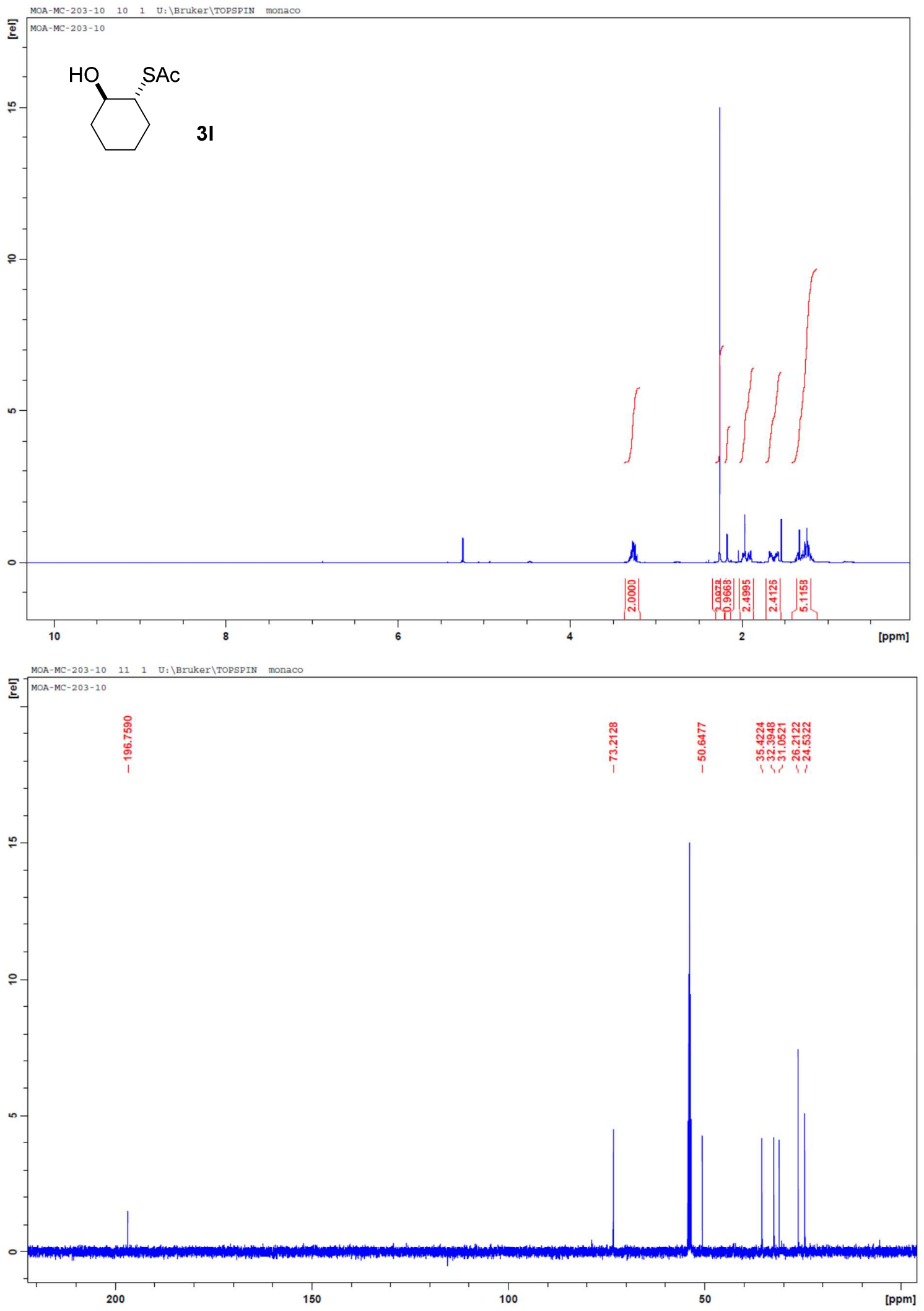

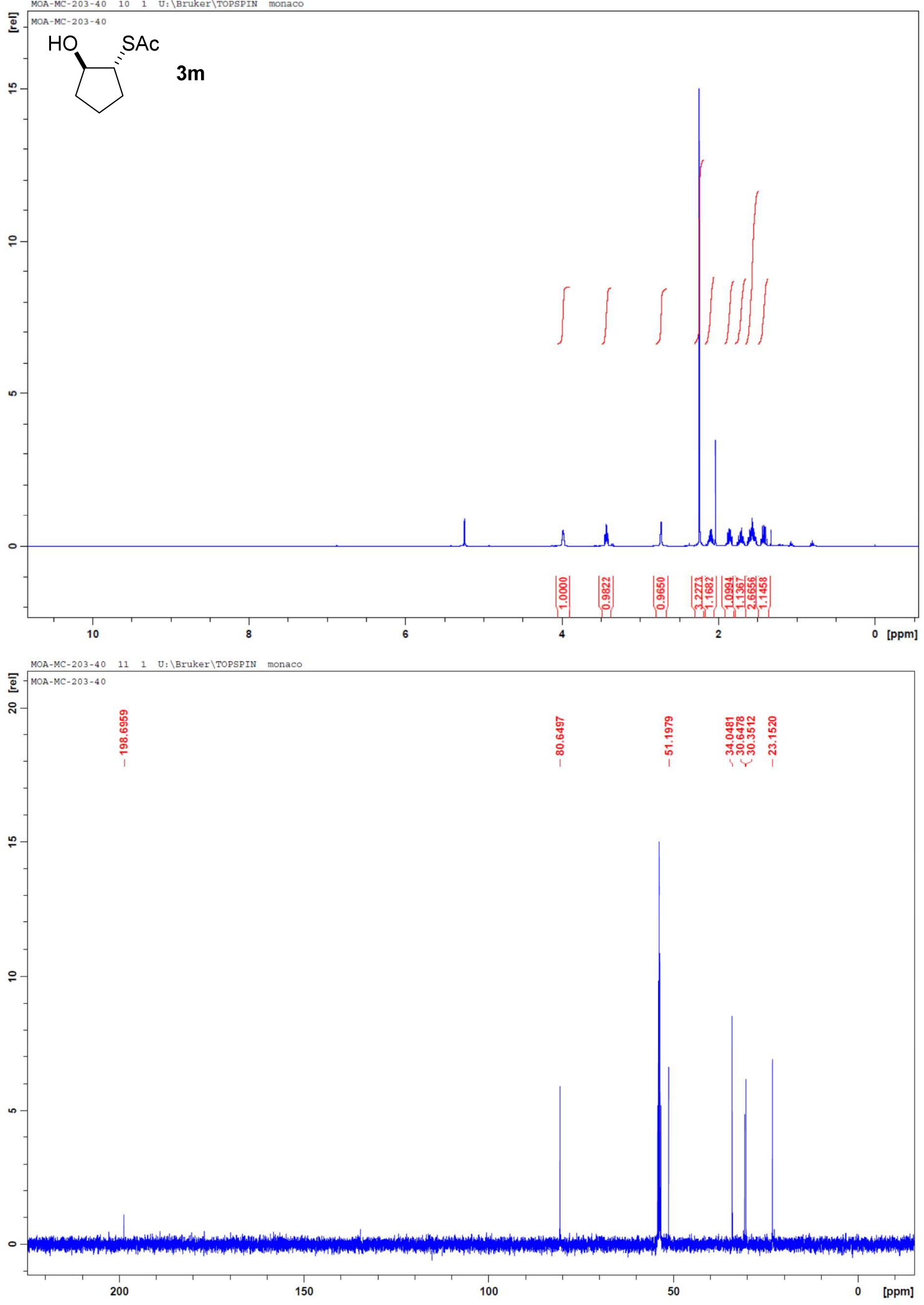

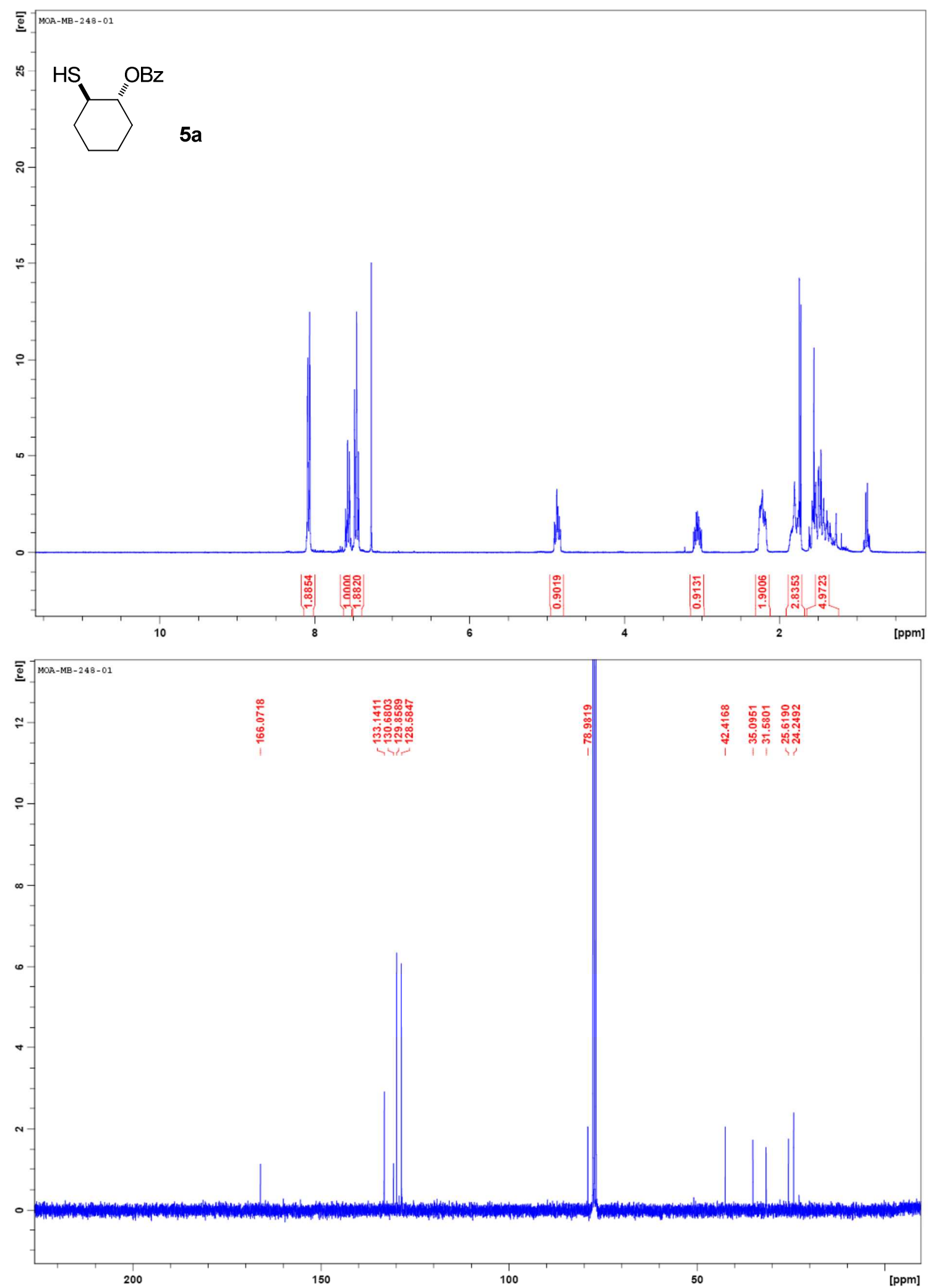

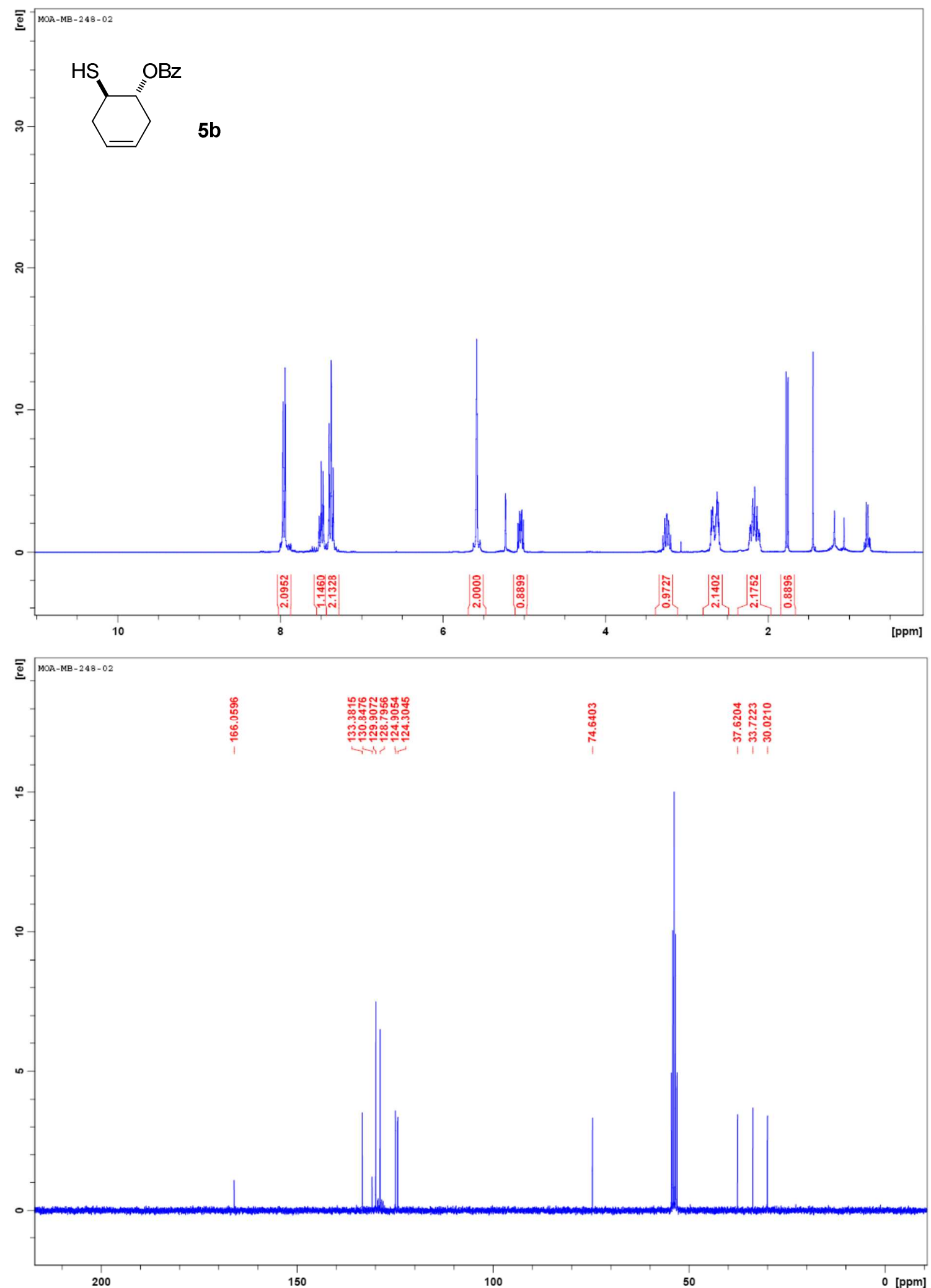

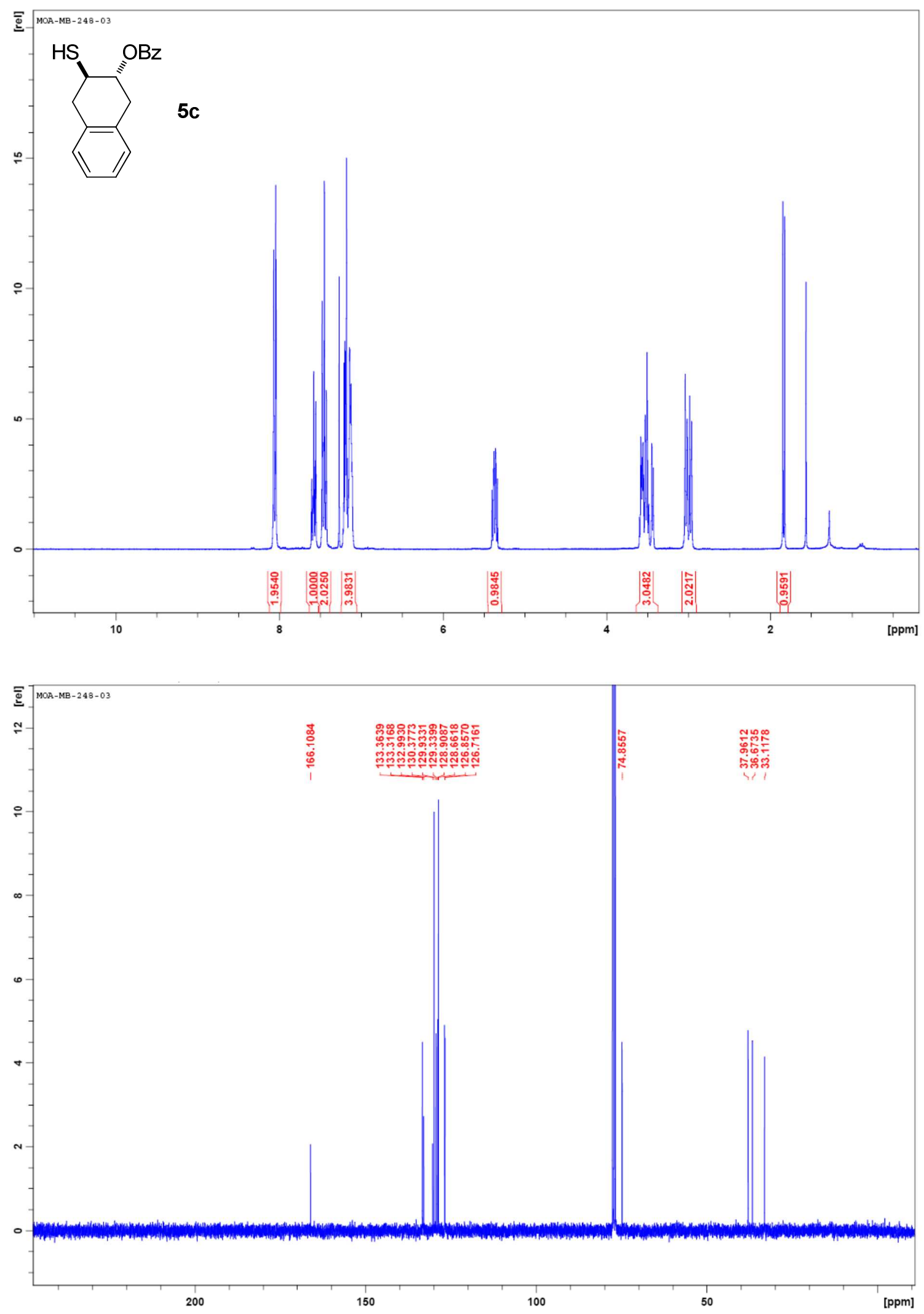

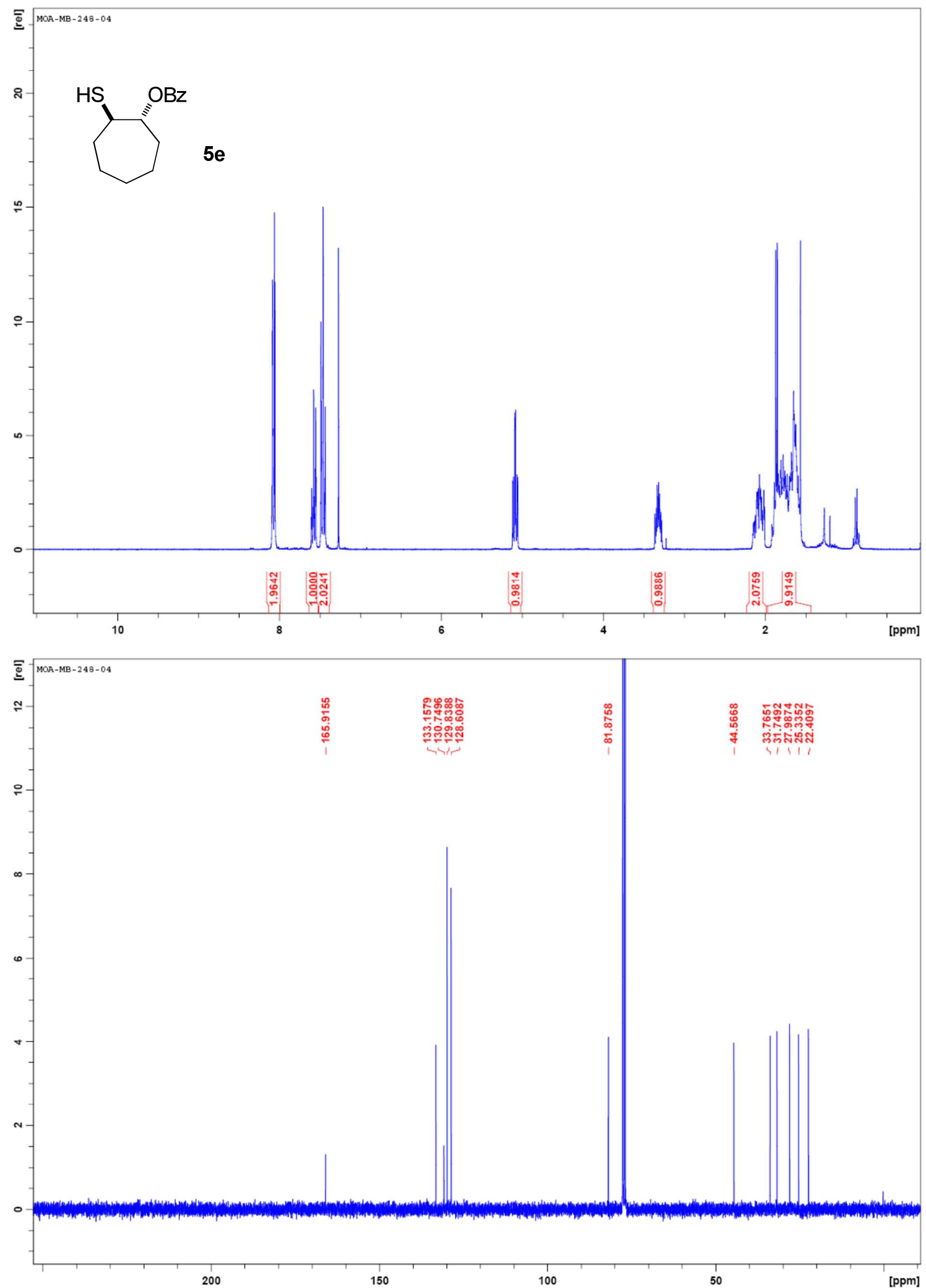

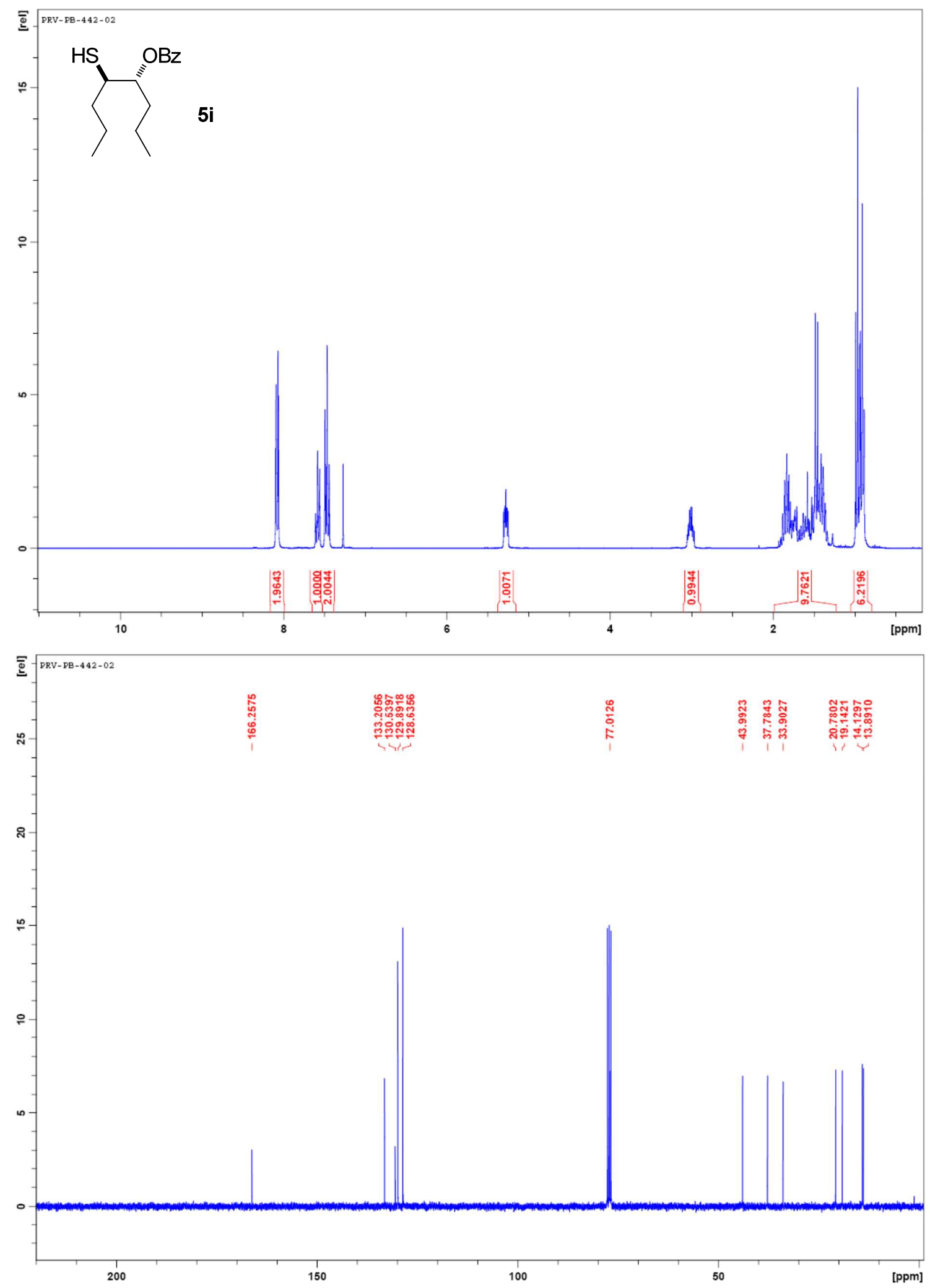

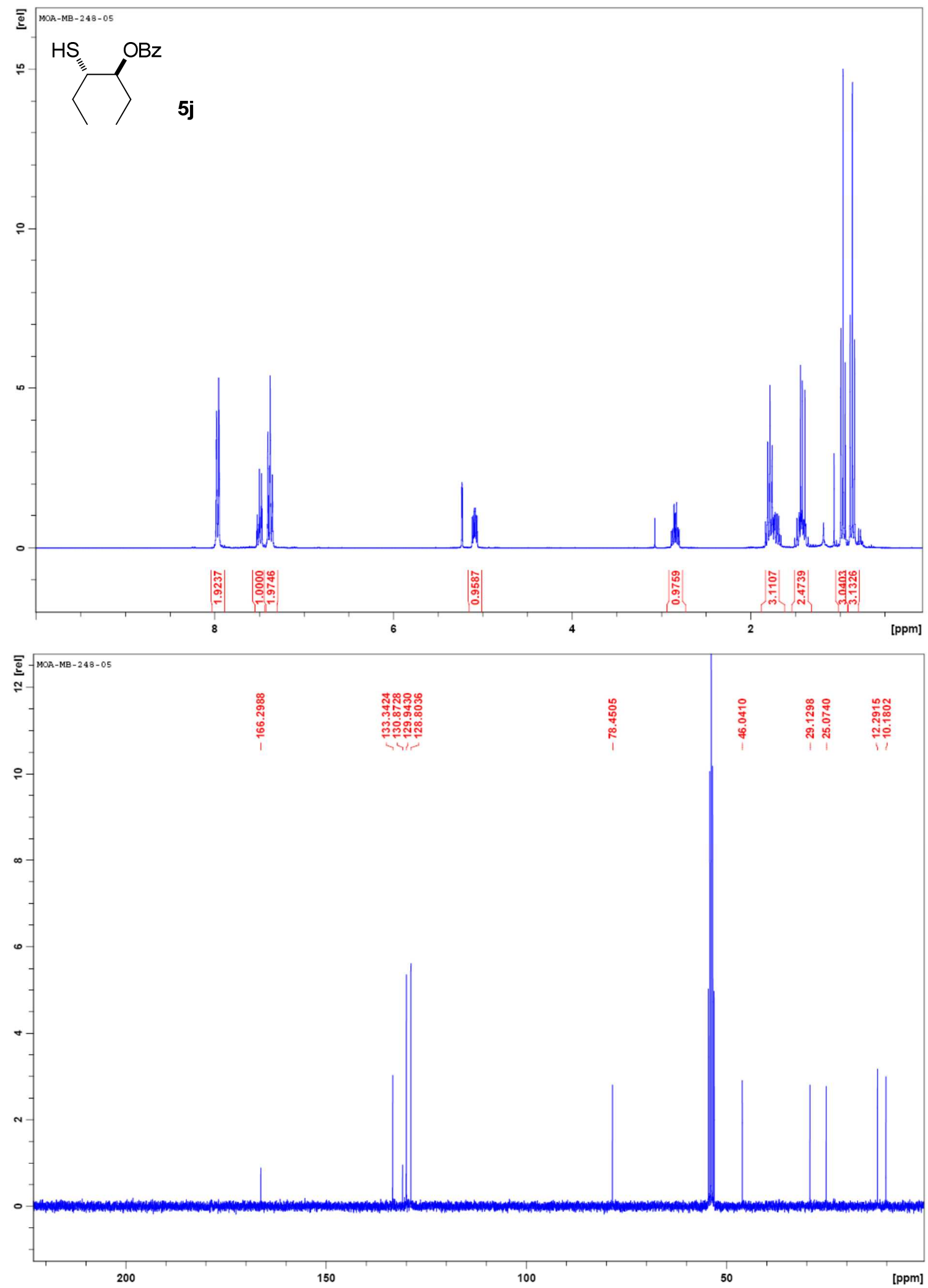

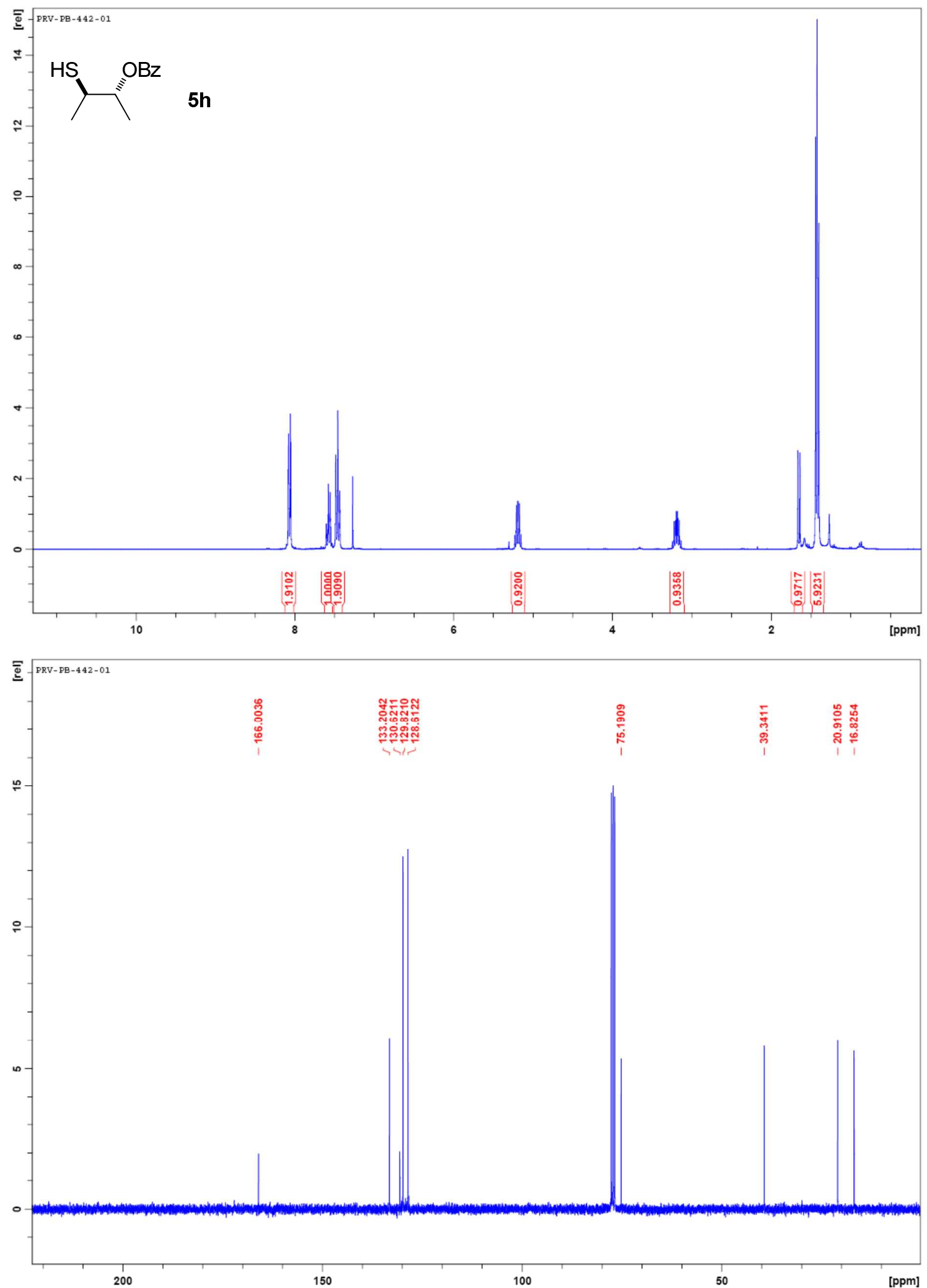

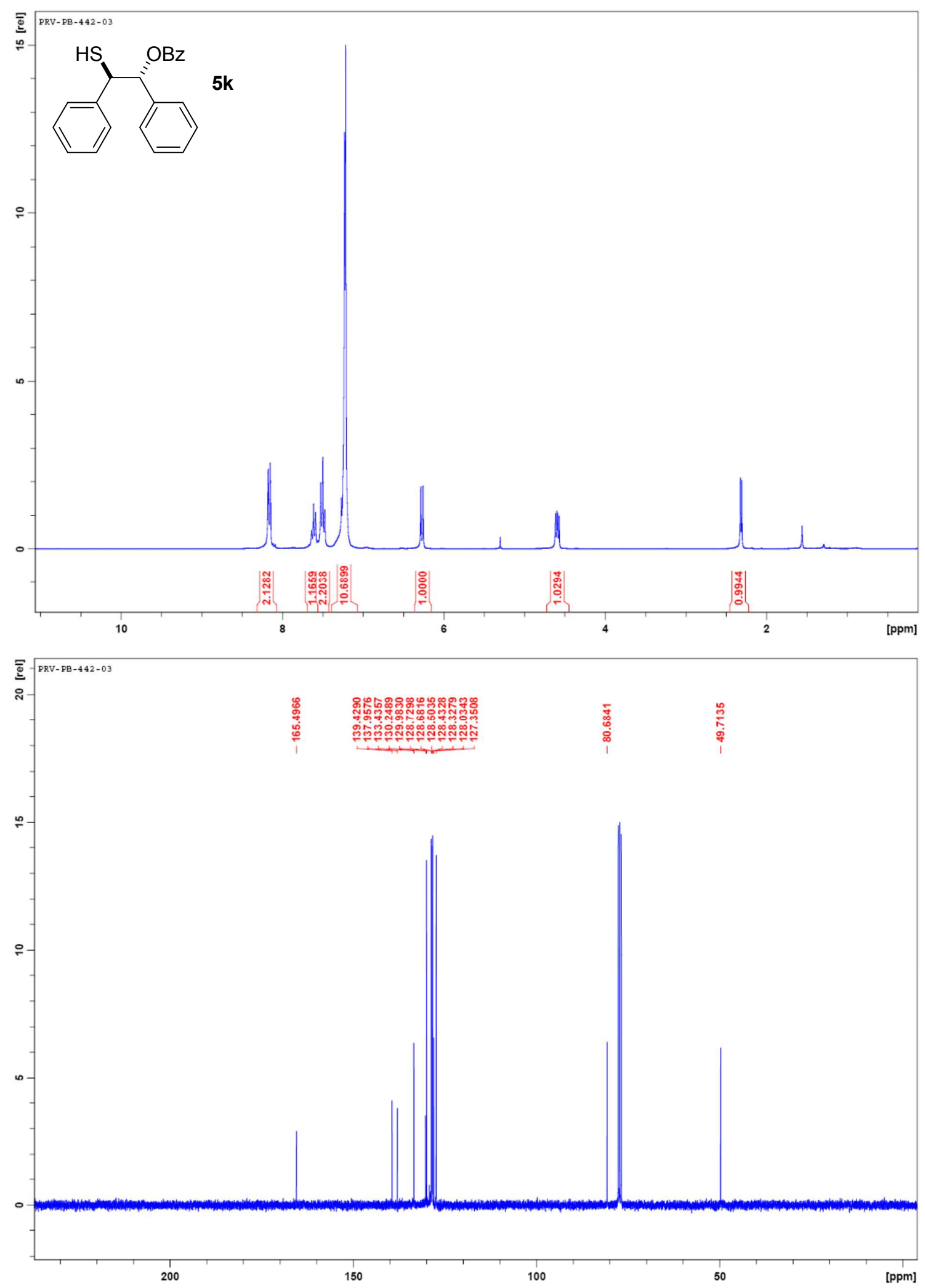

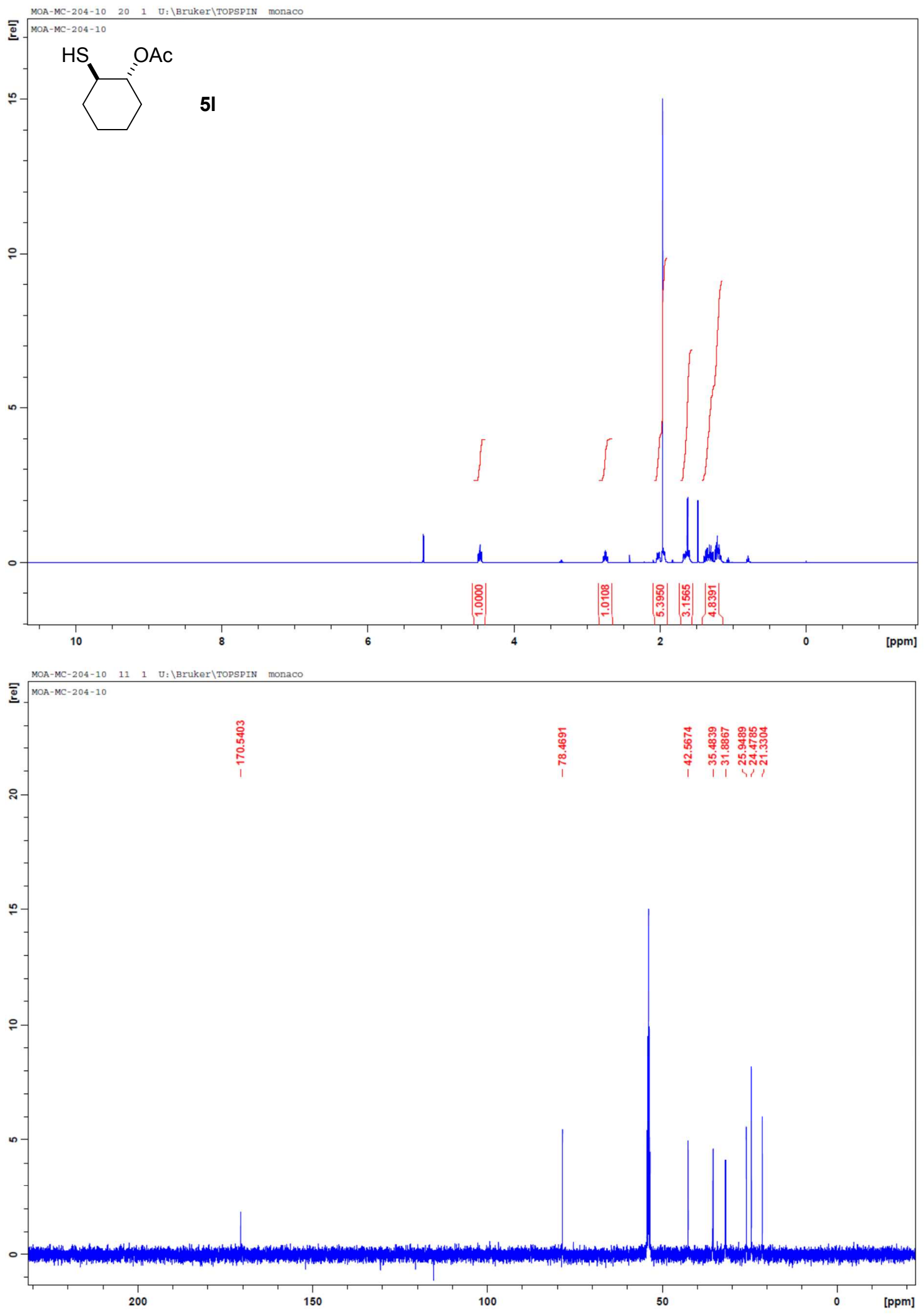

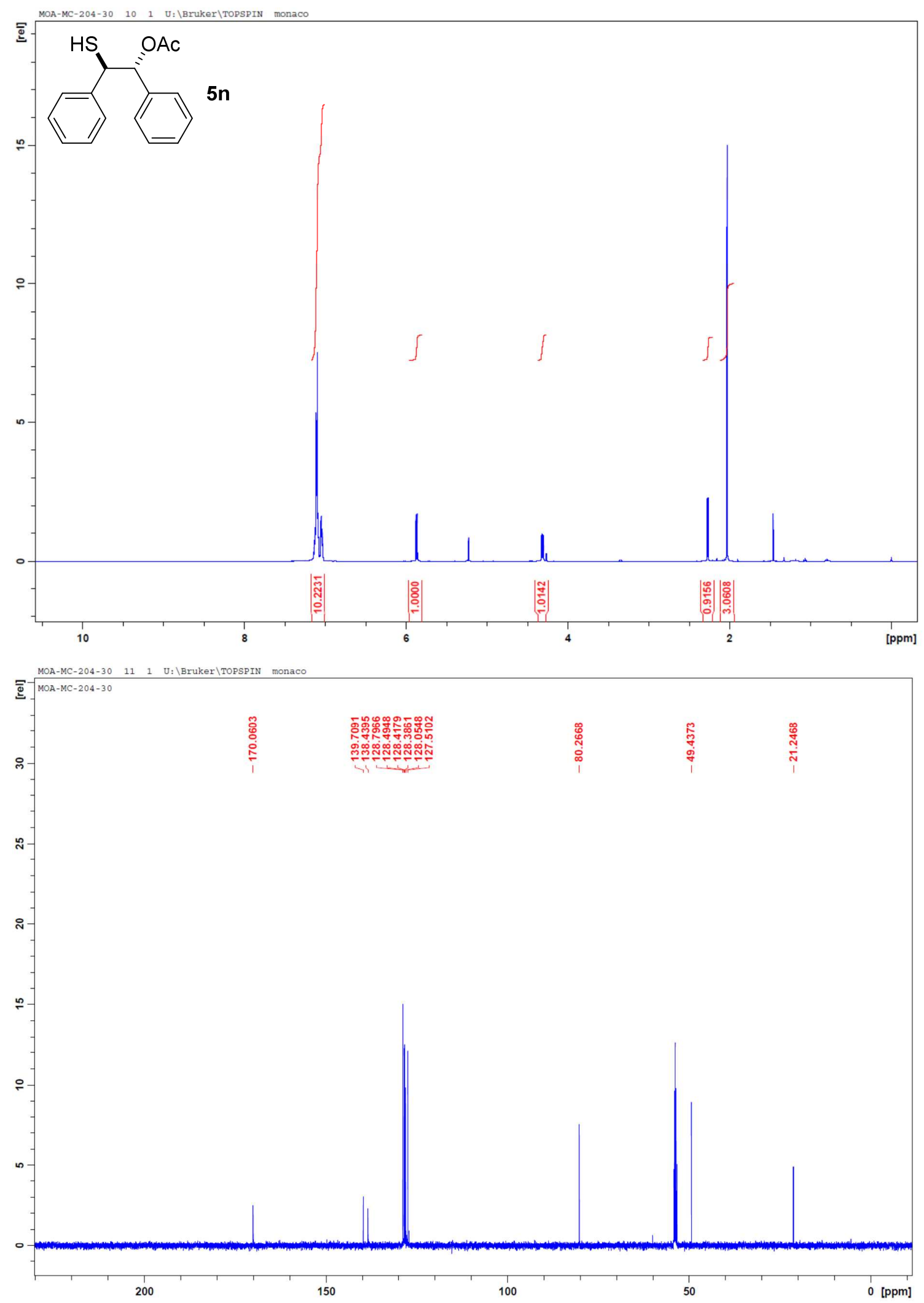

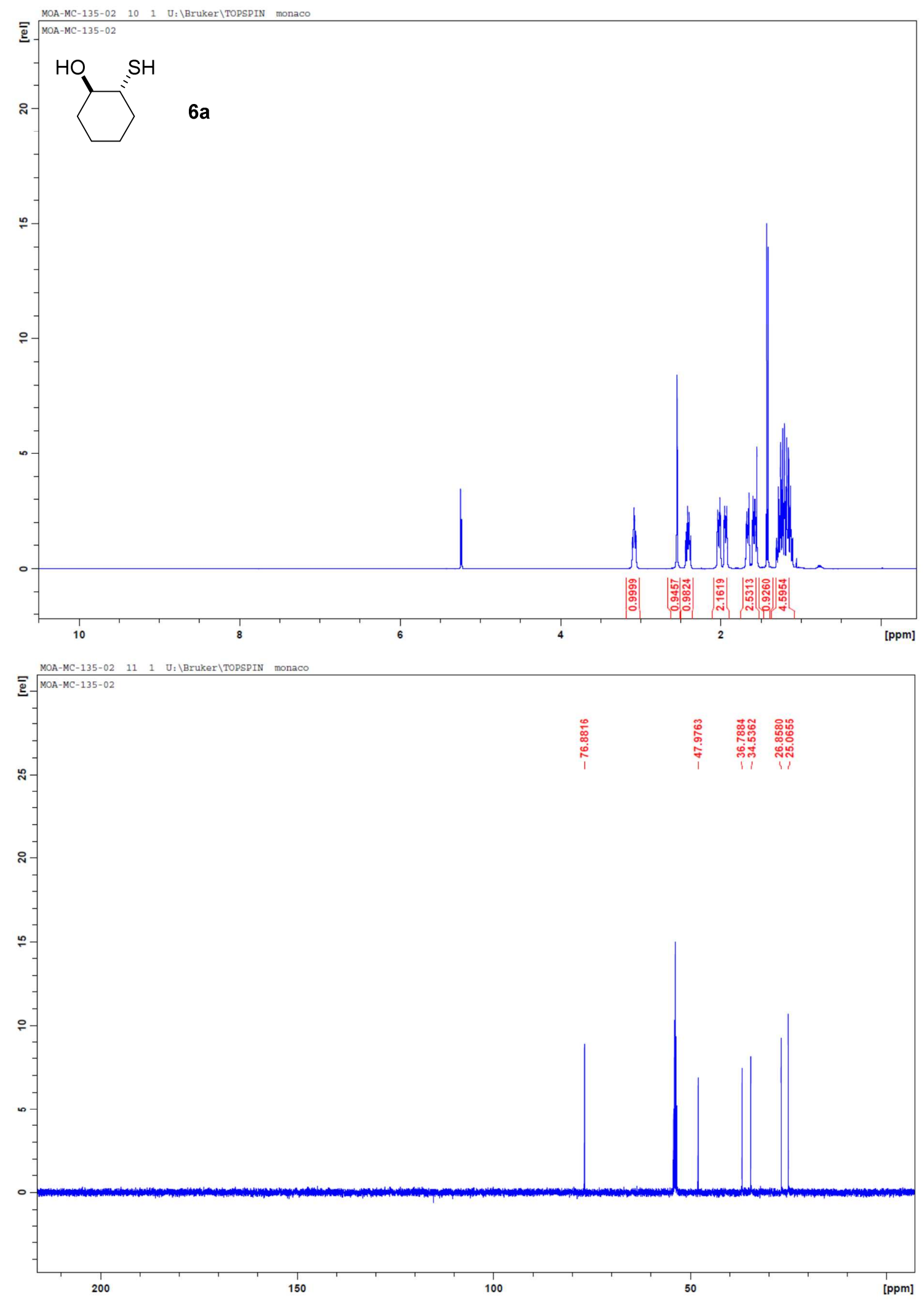

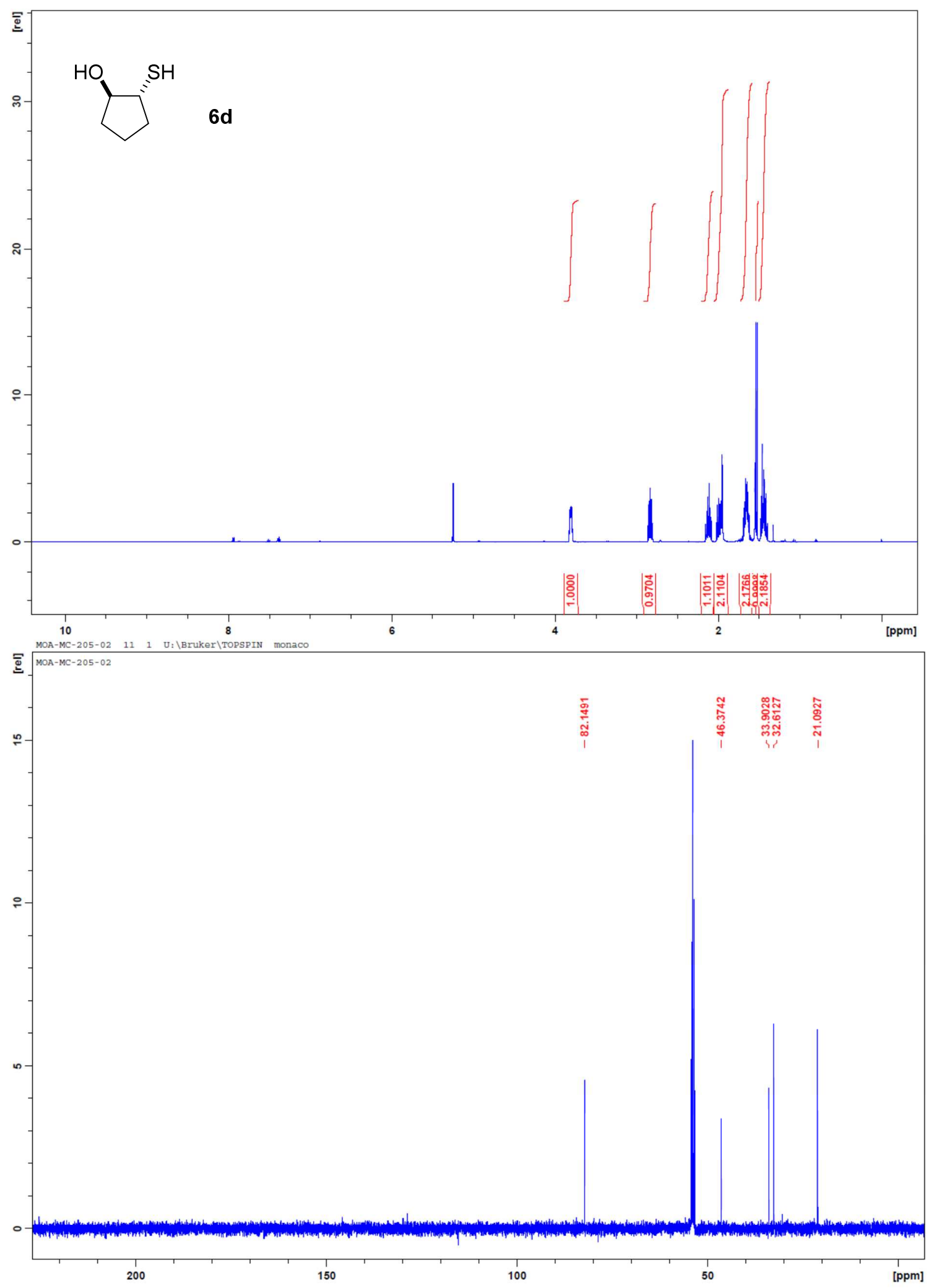

S-56 


\section{HPLC and GC traces}
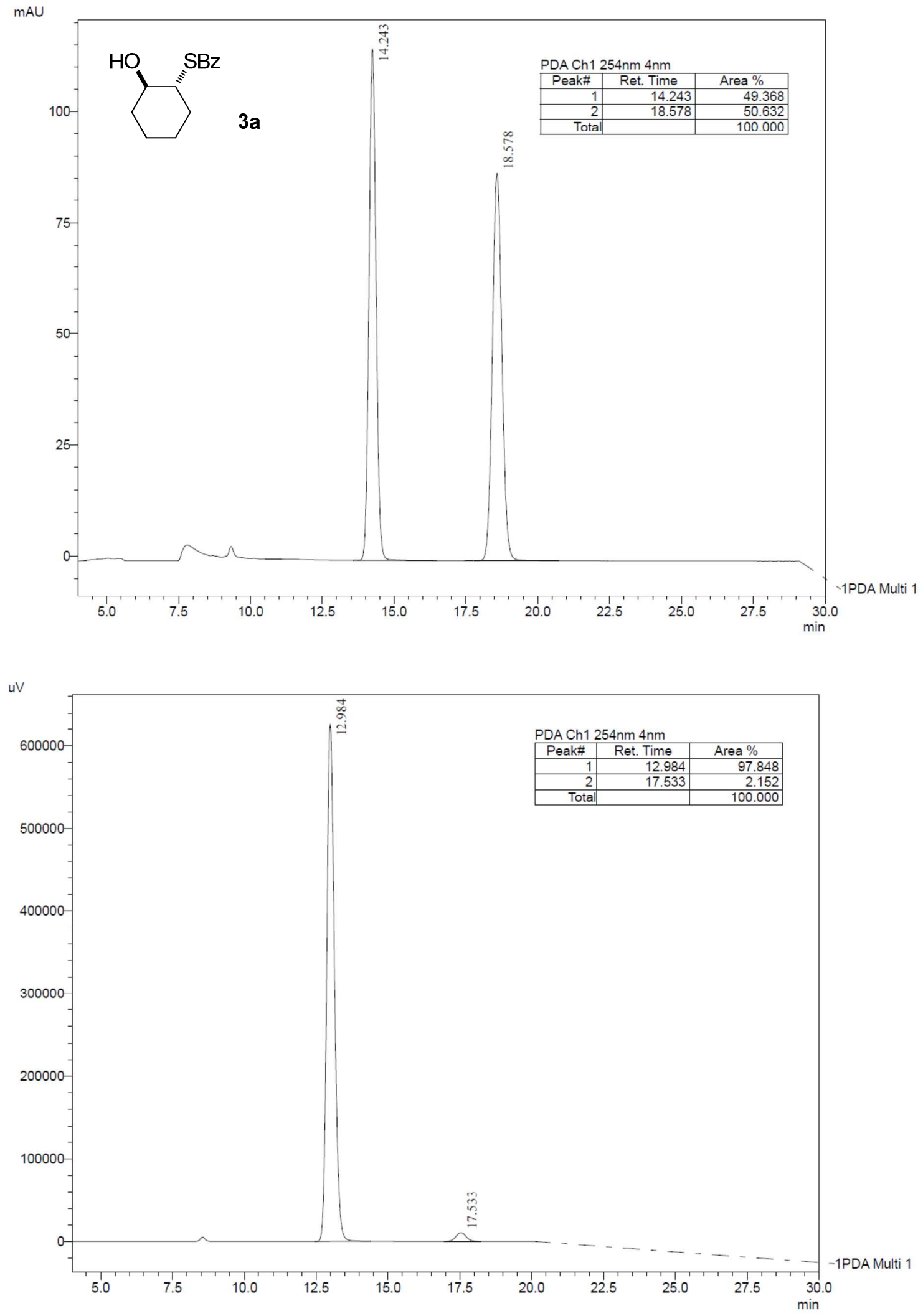

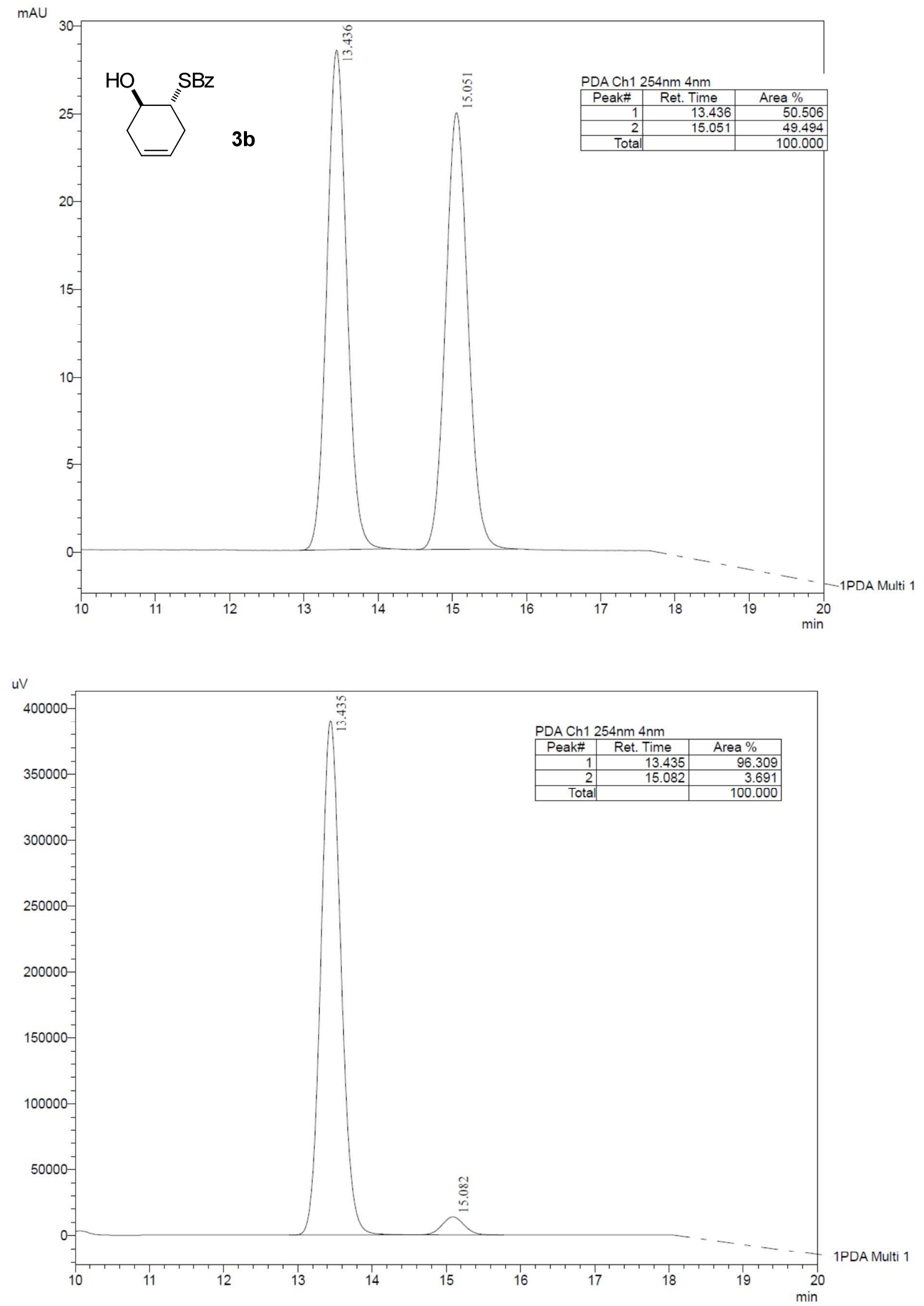

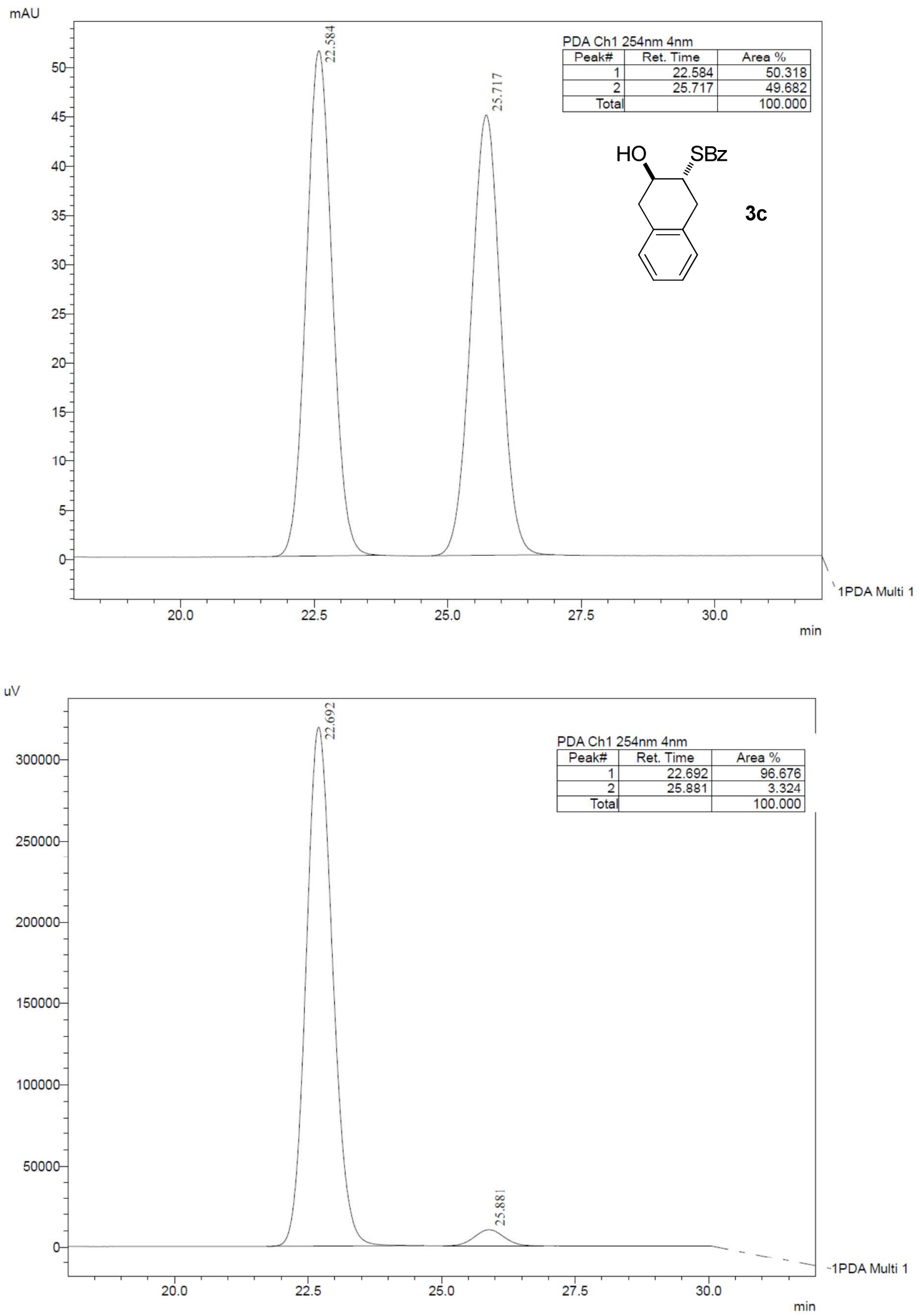

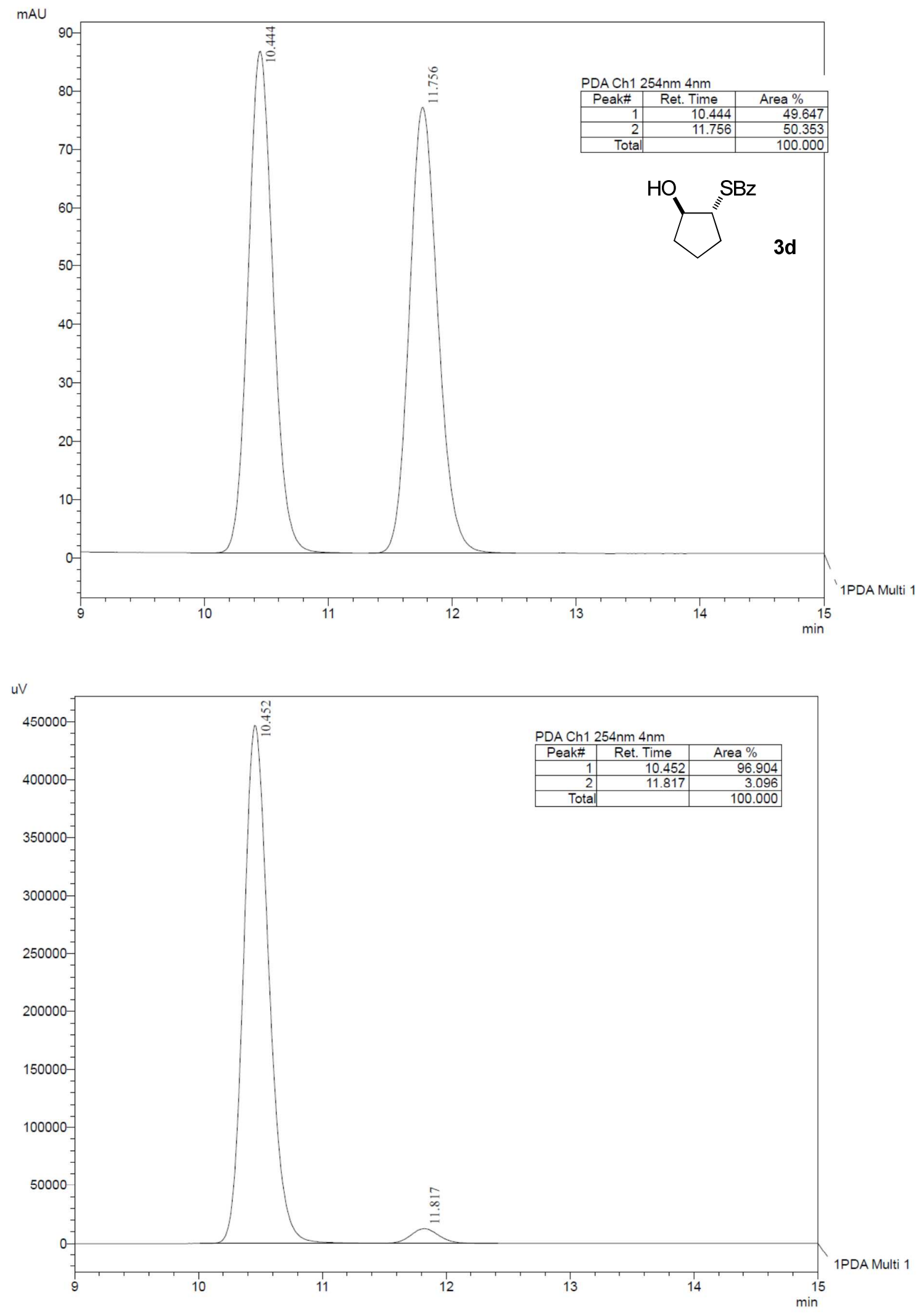

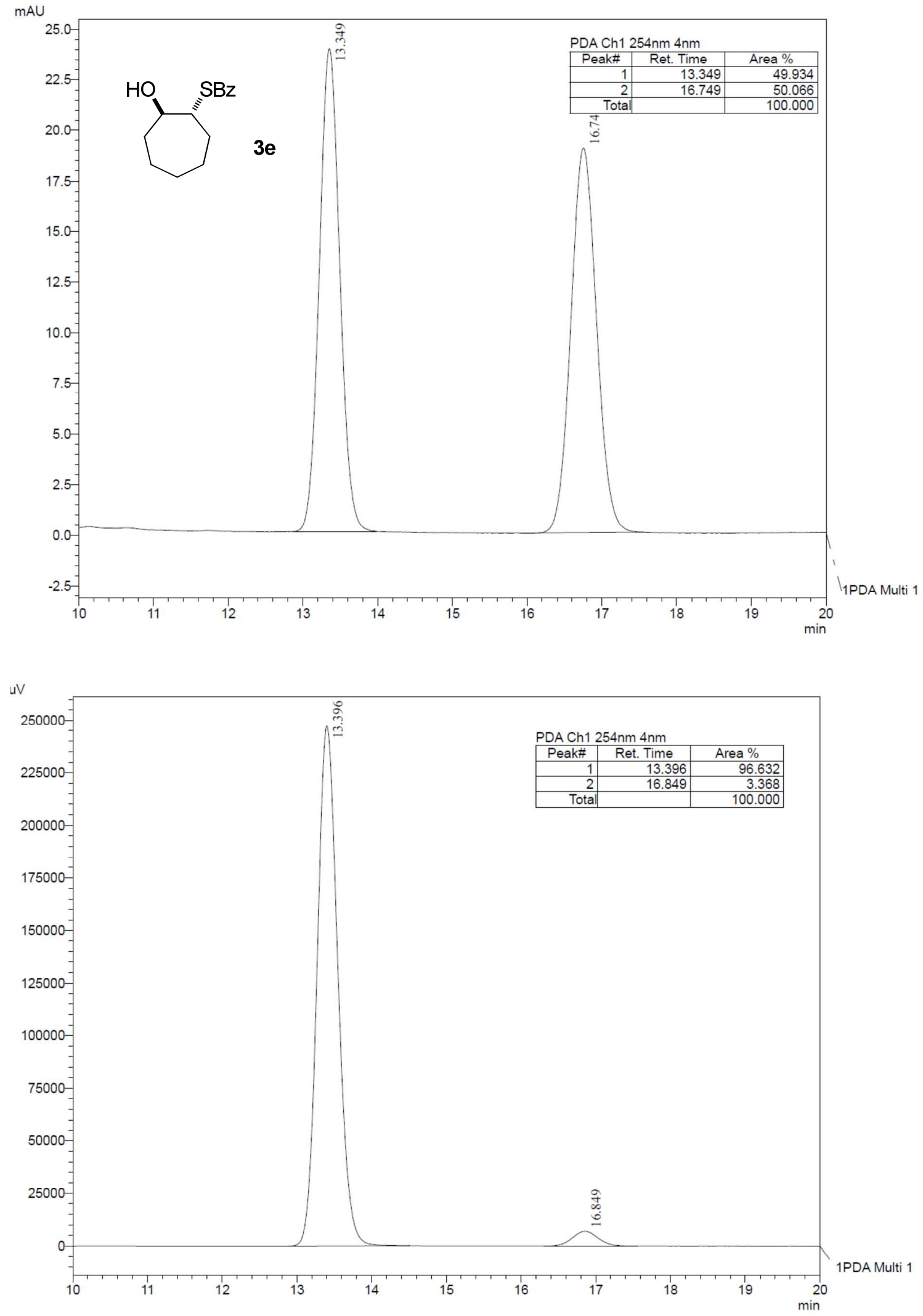

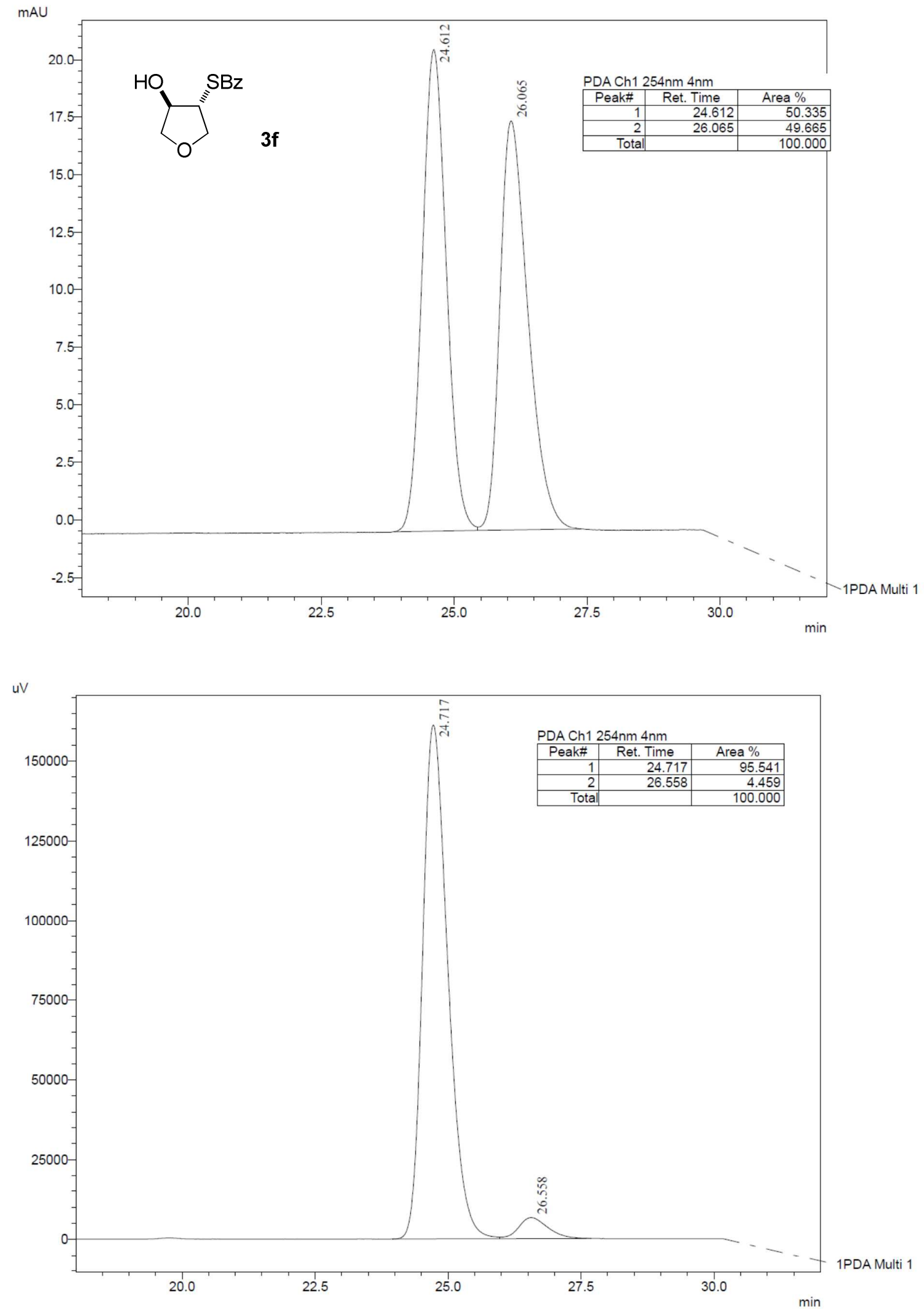

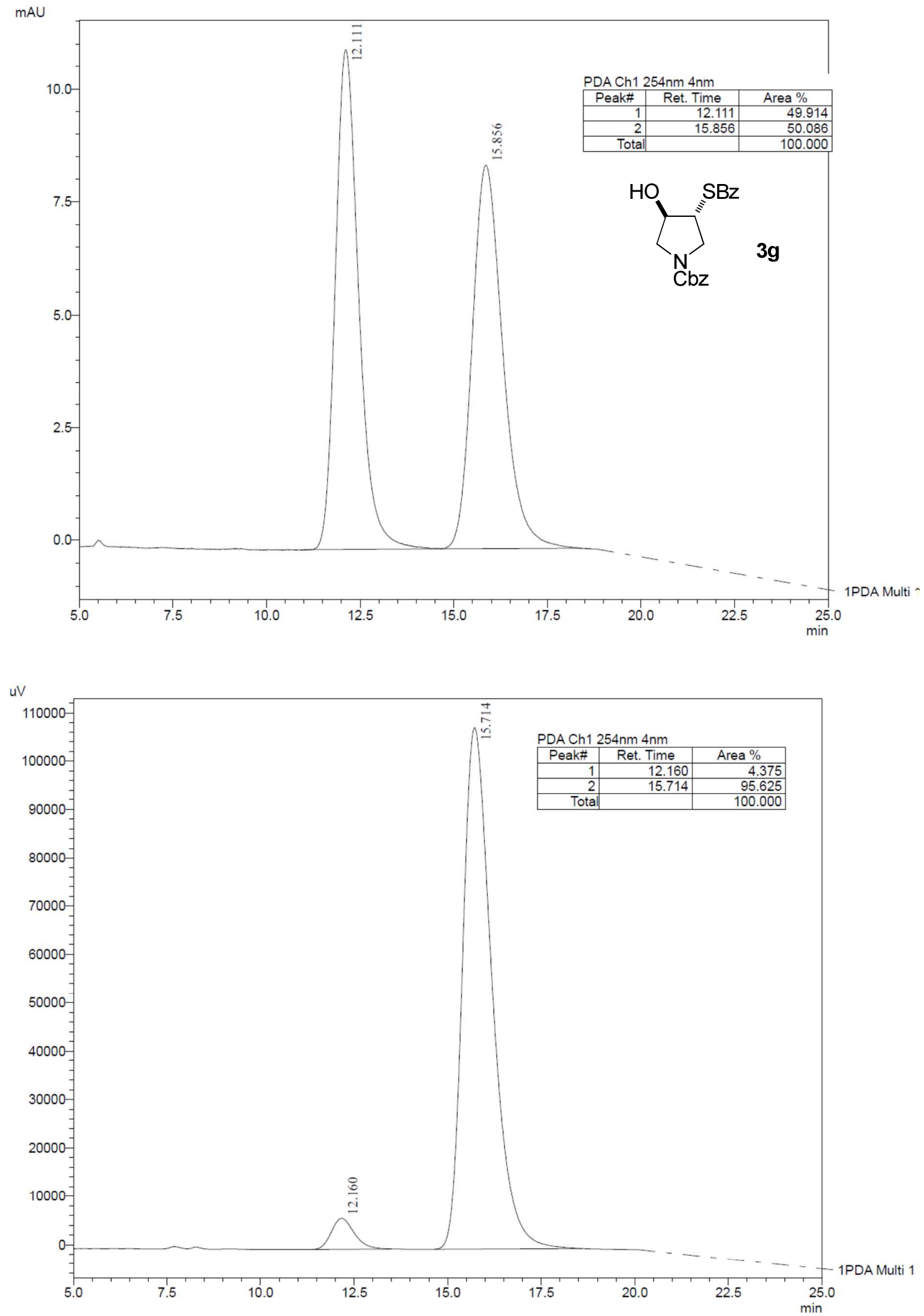

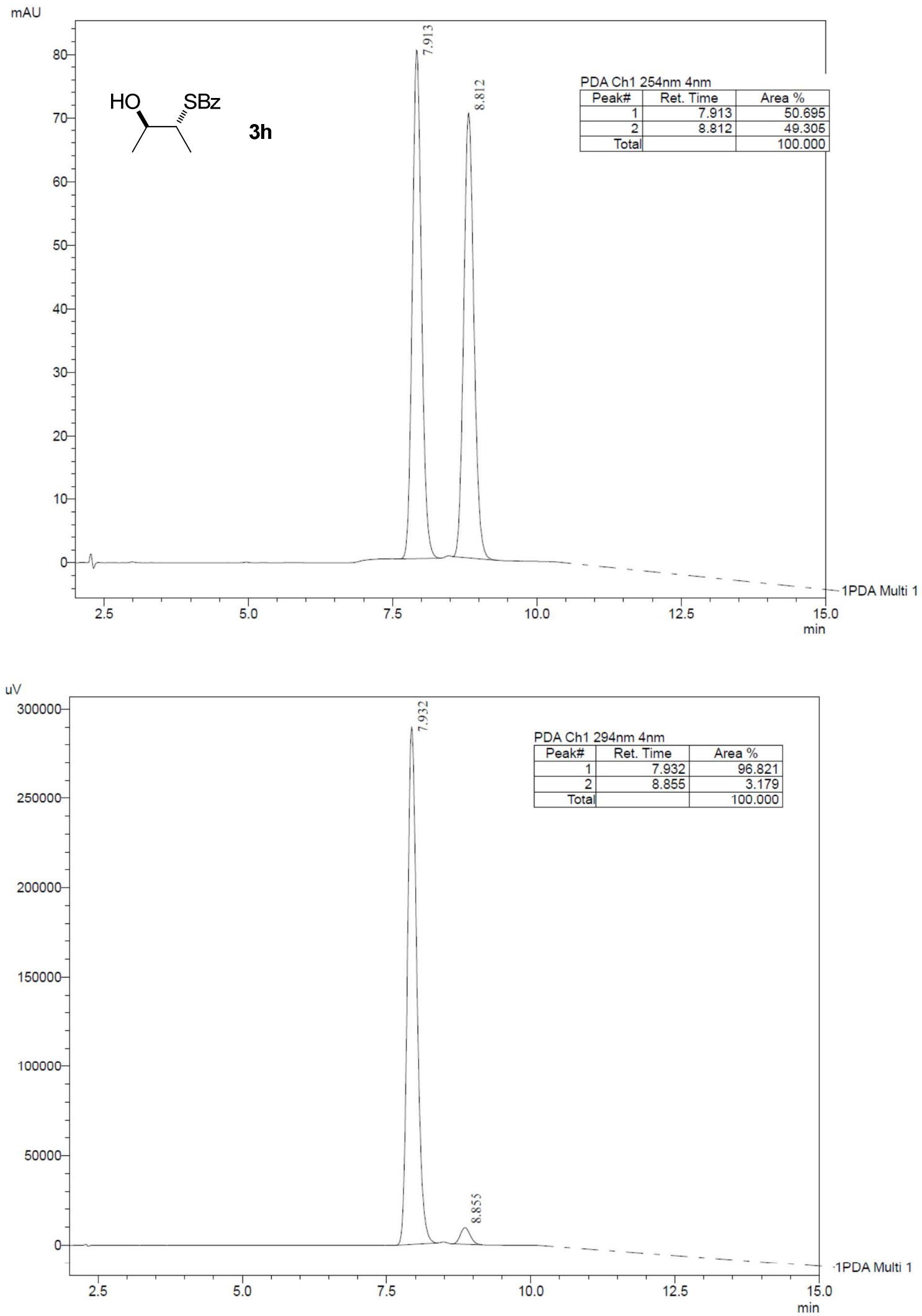

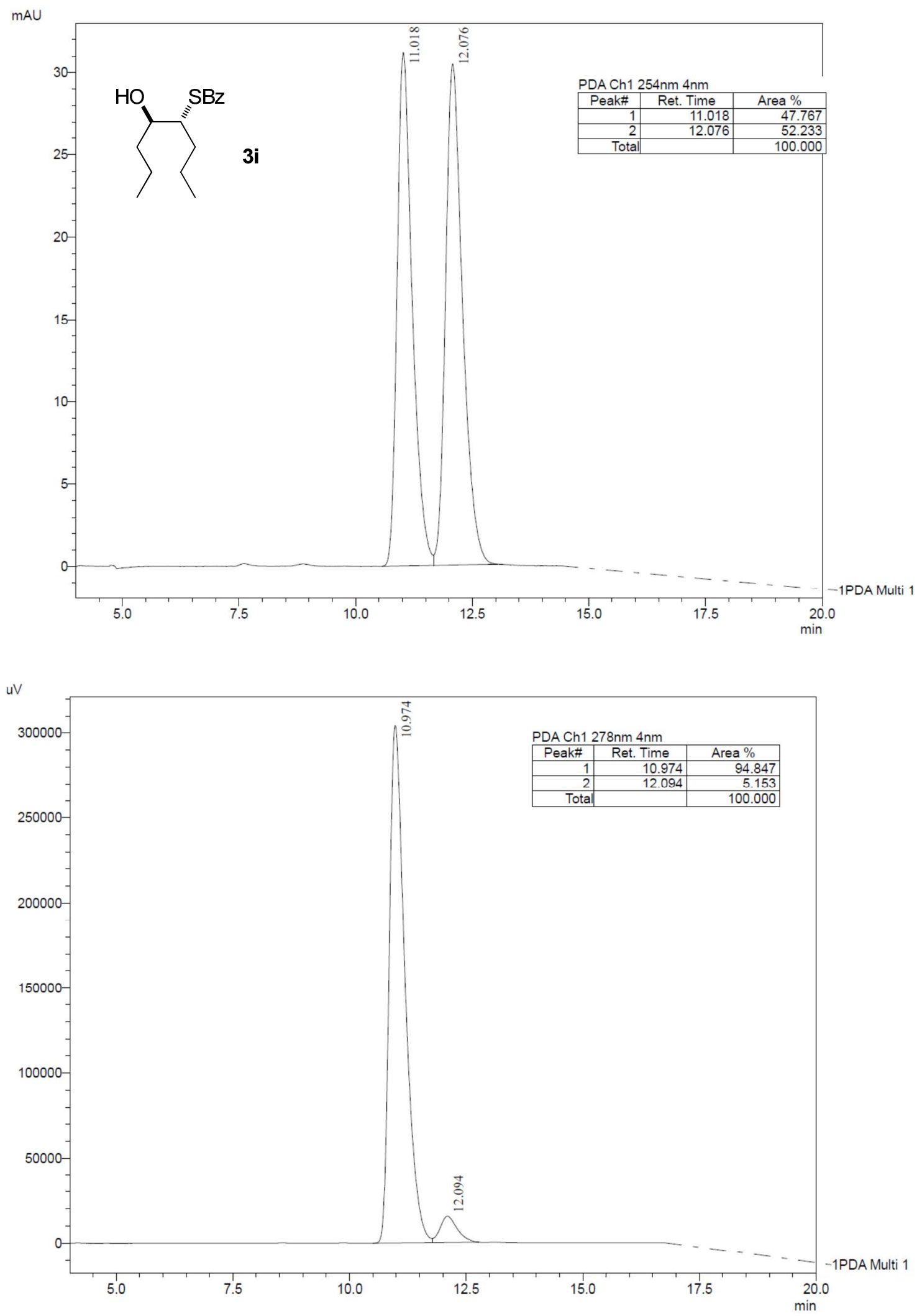

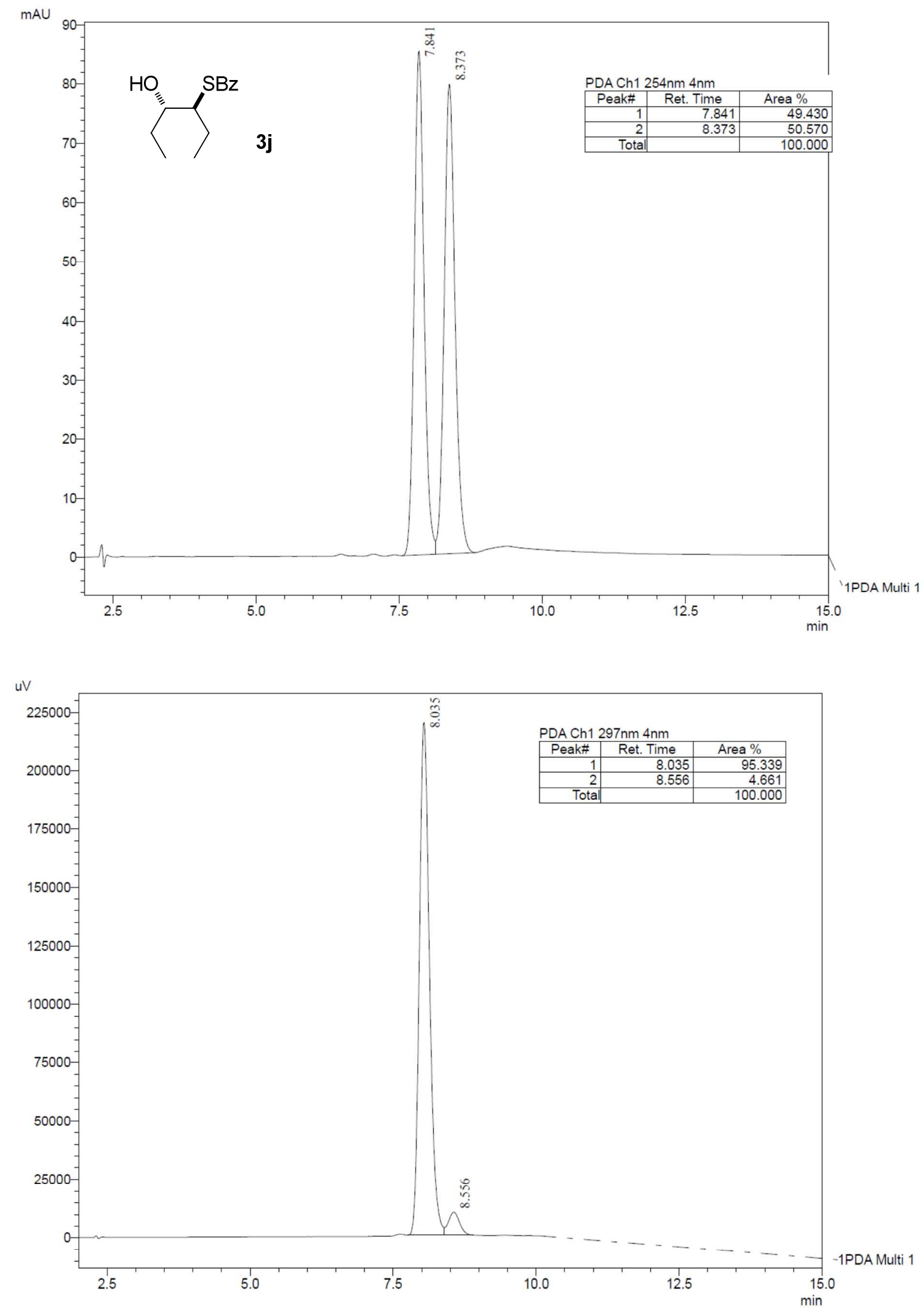

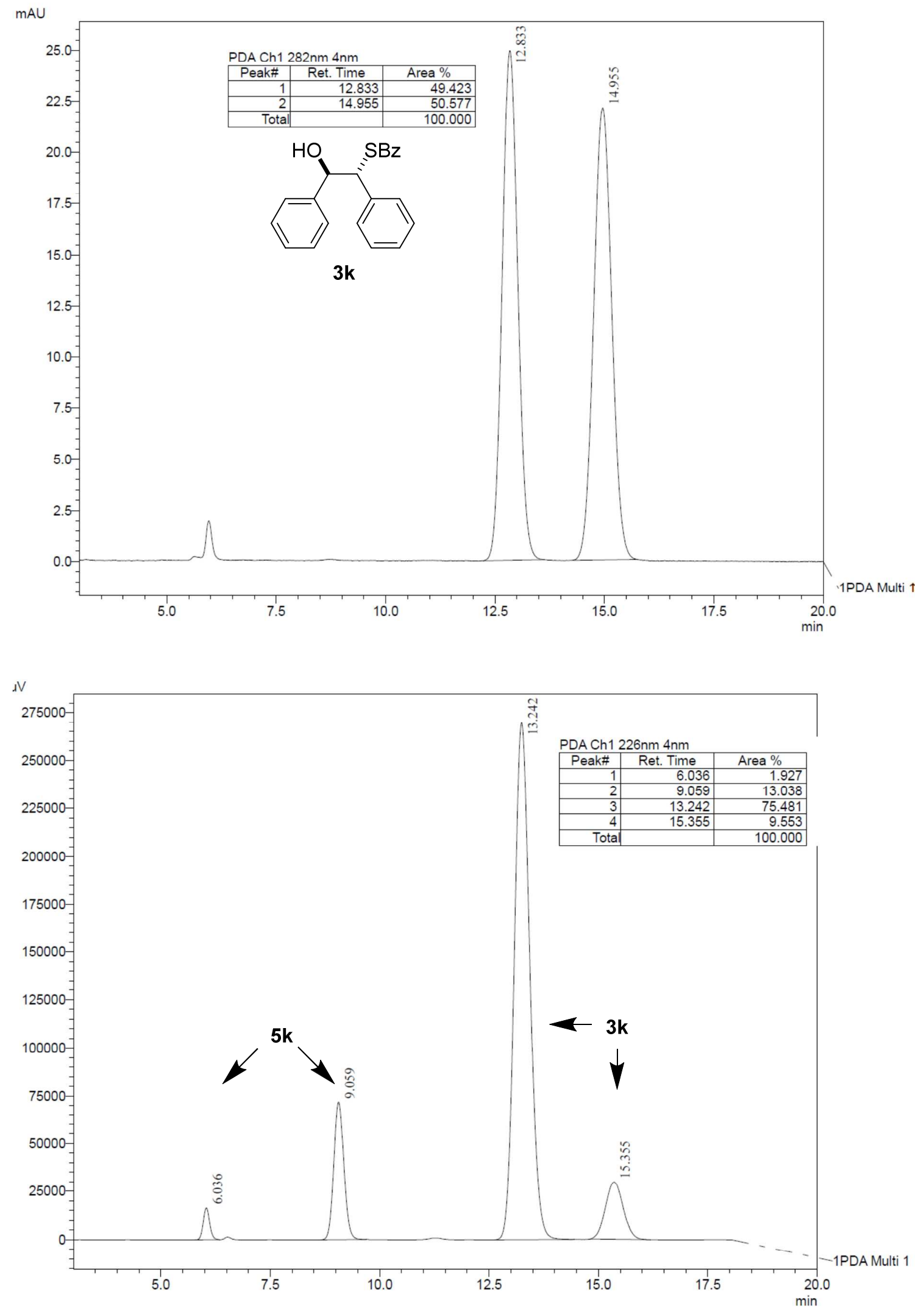


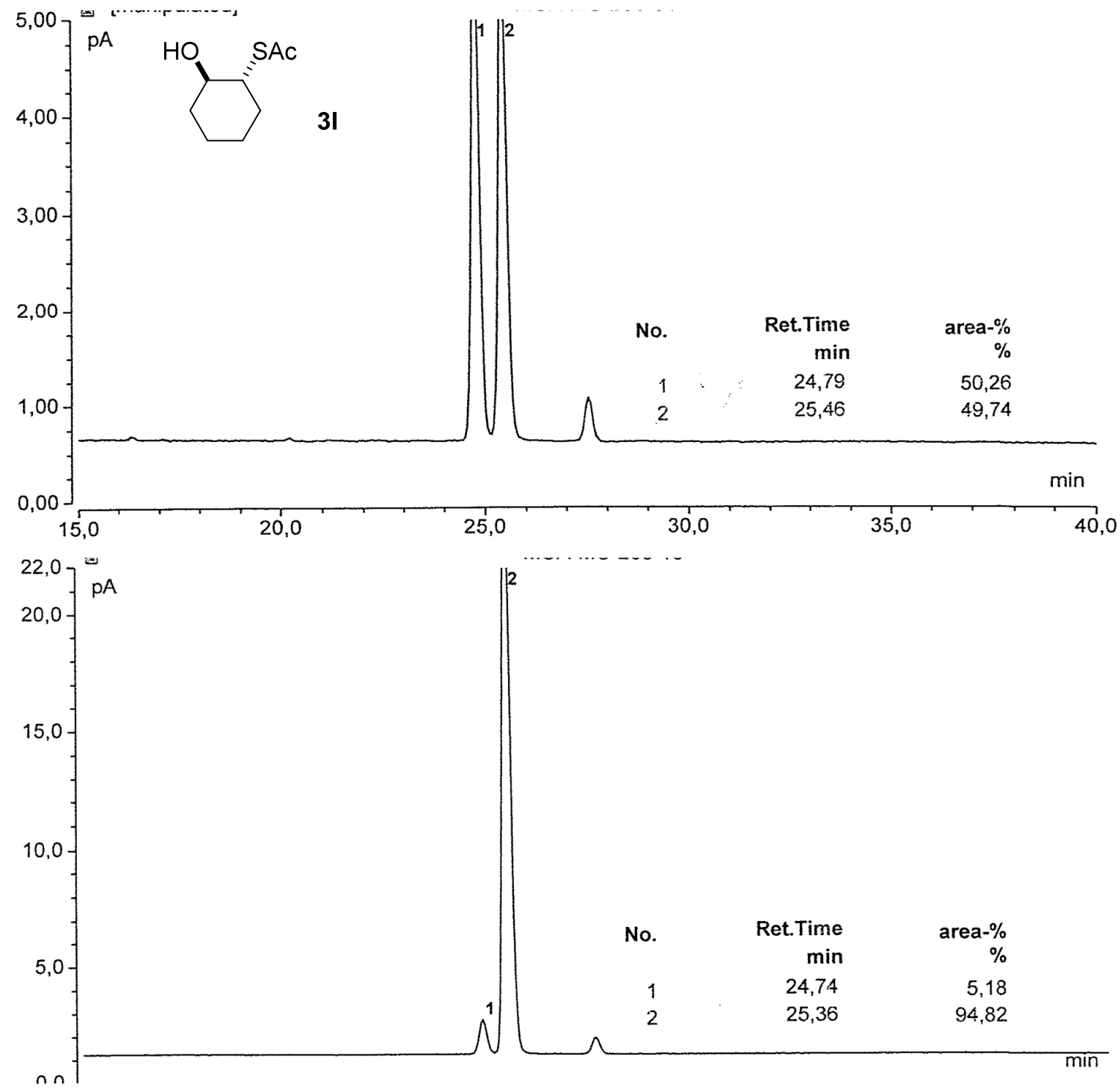



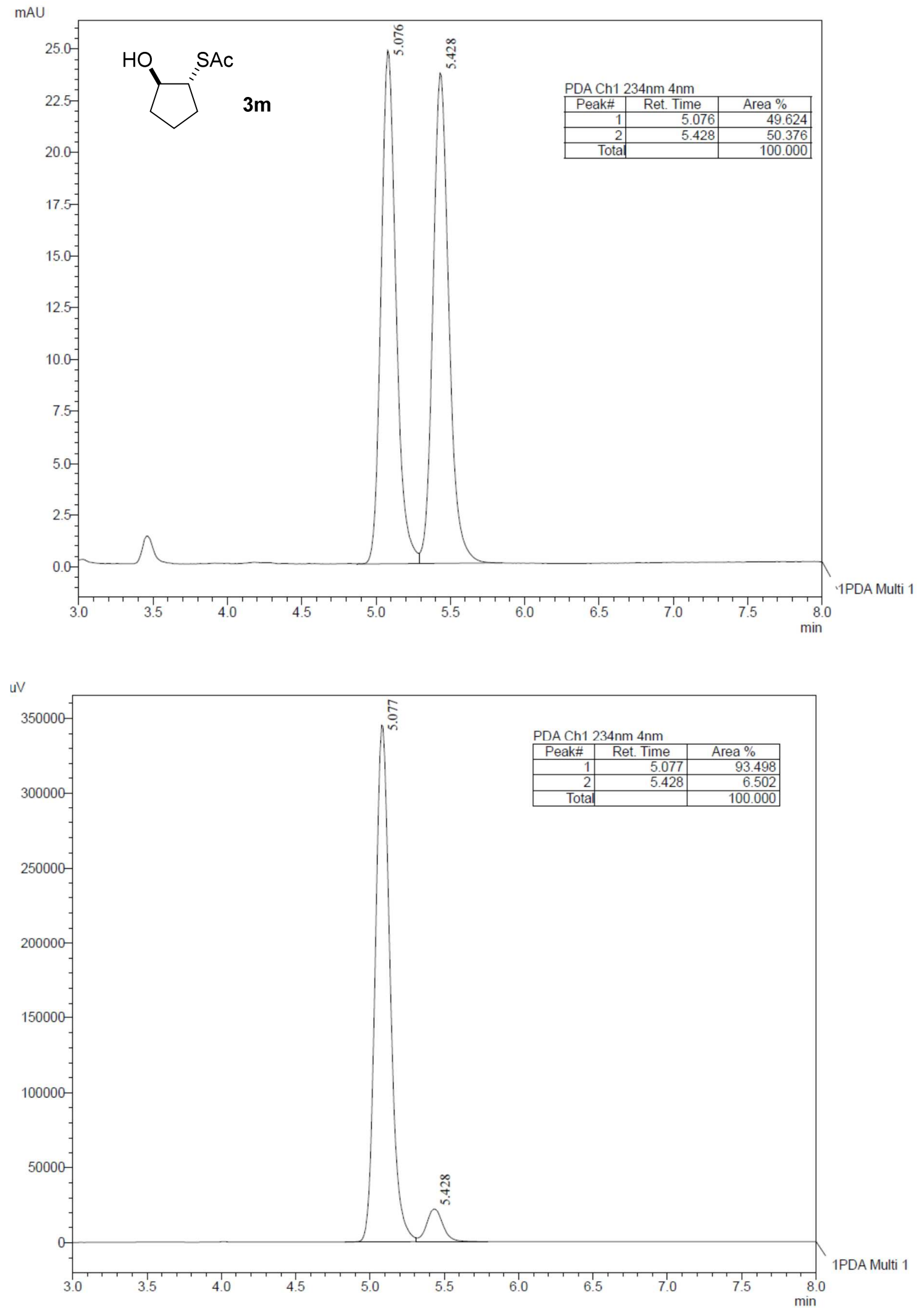

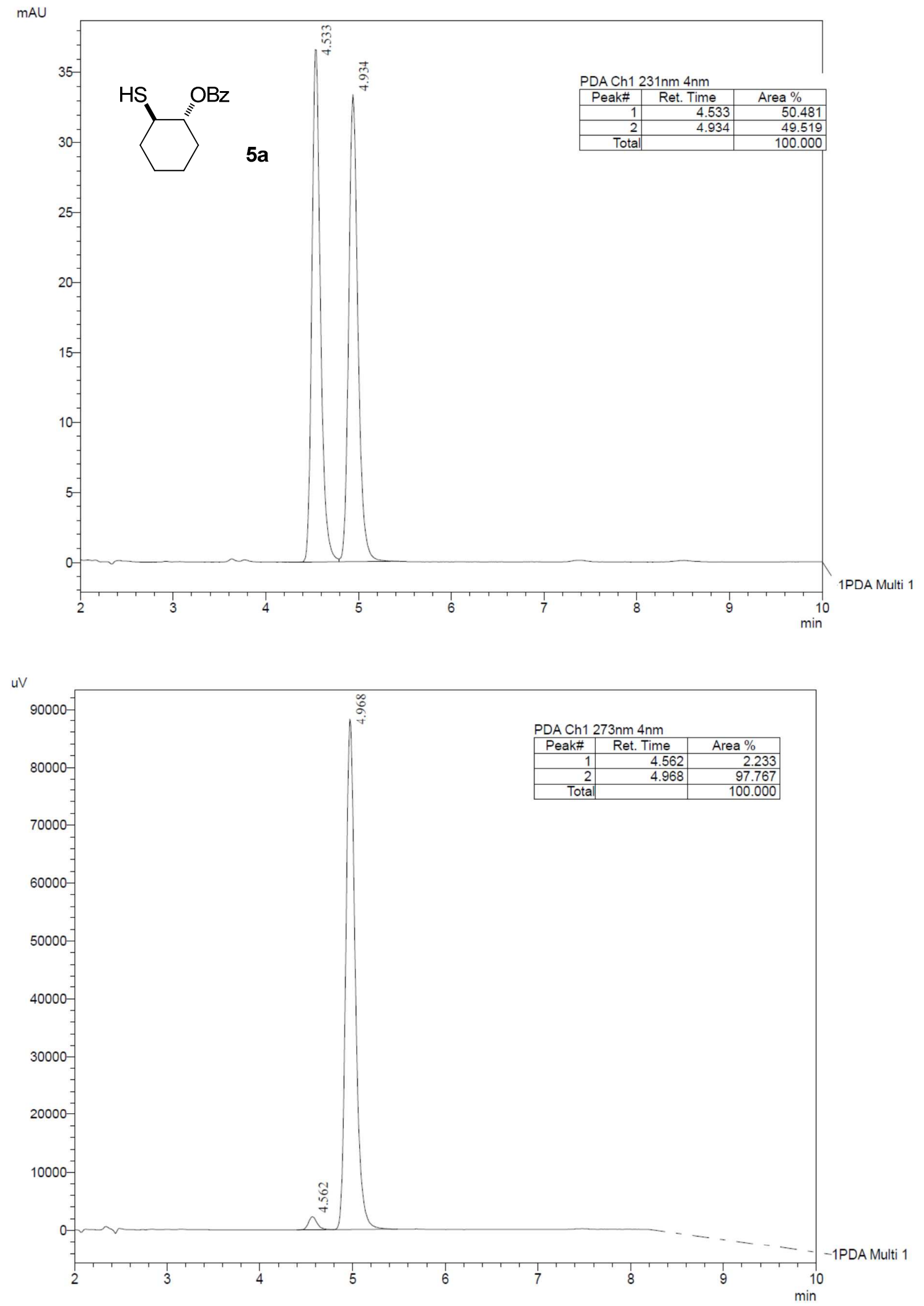

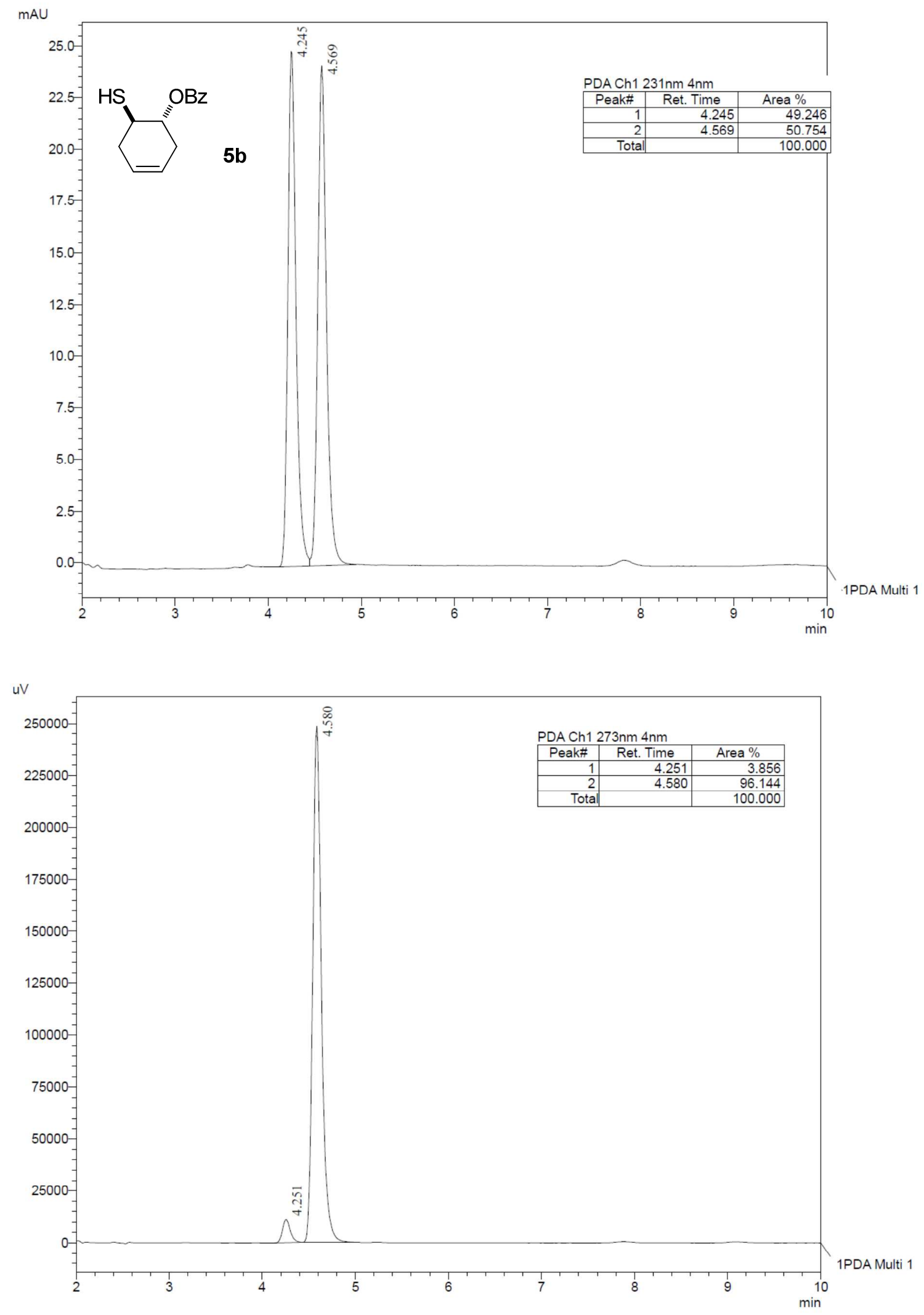

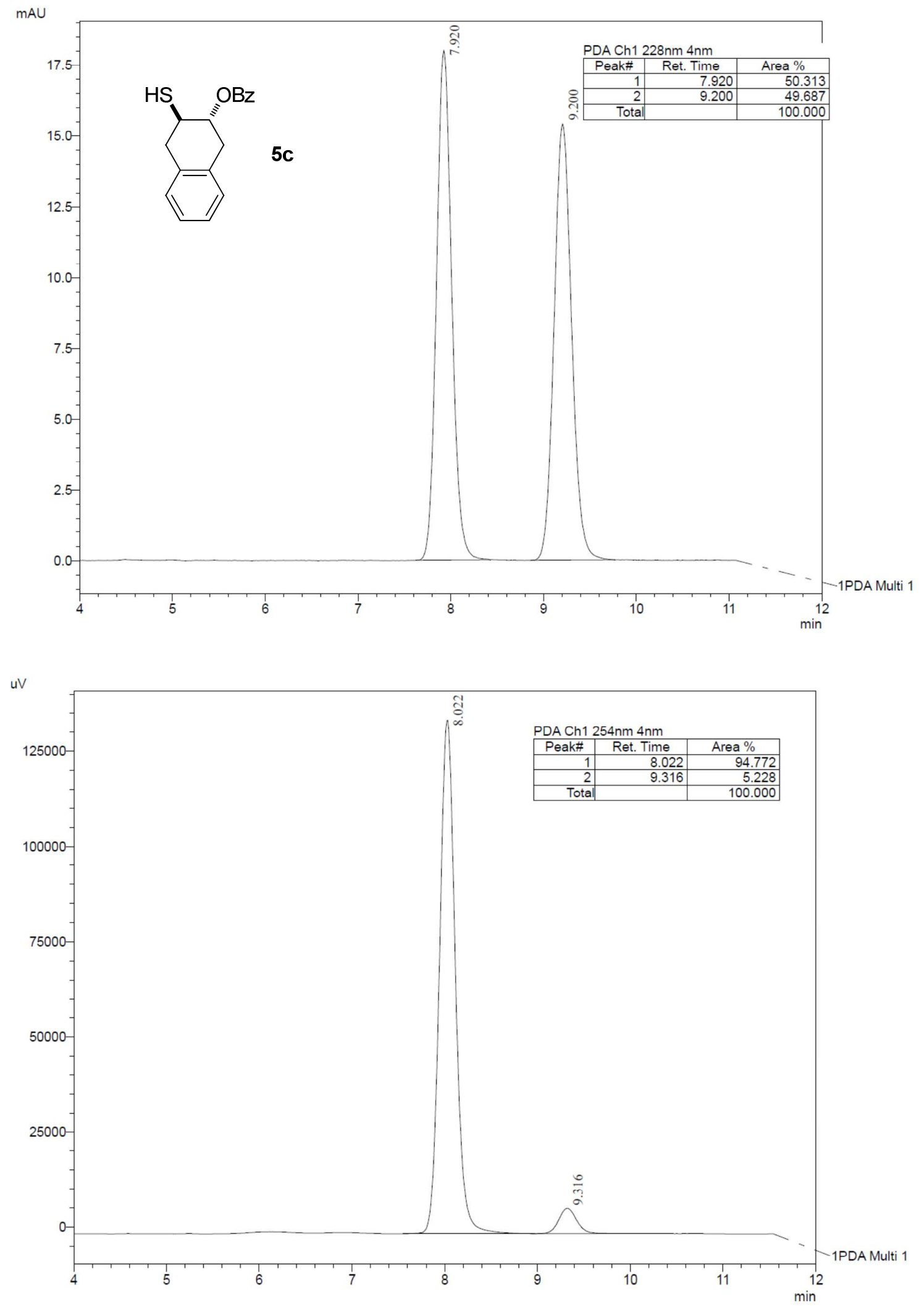

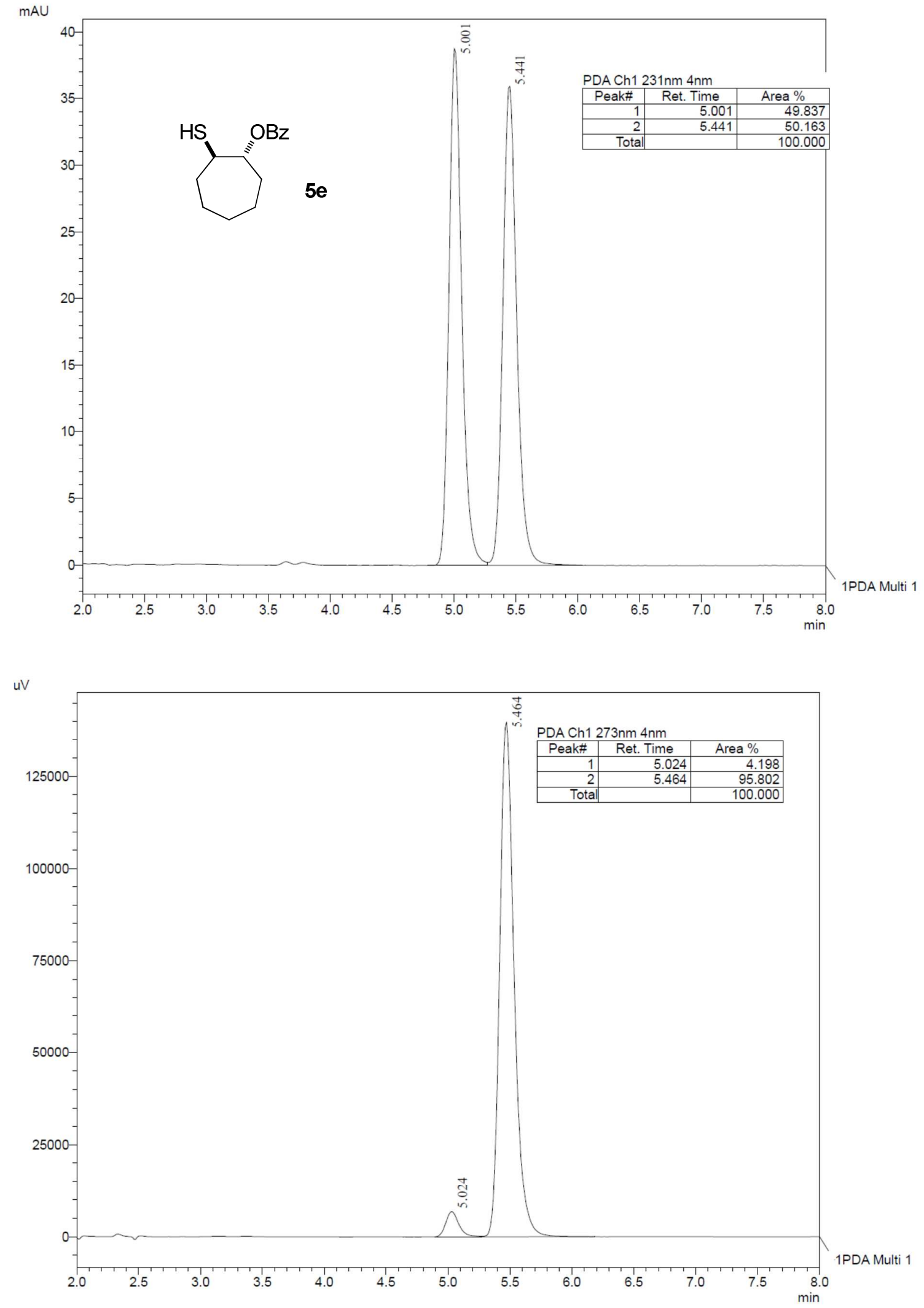

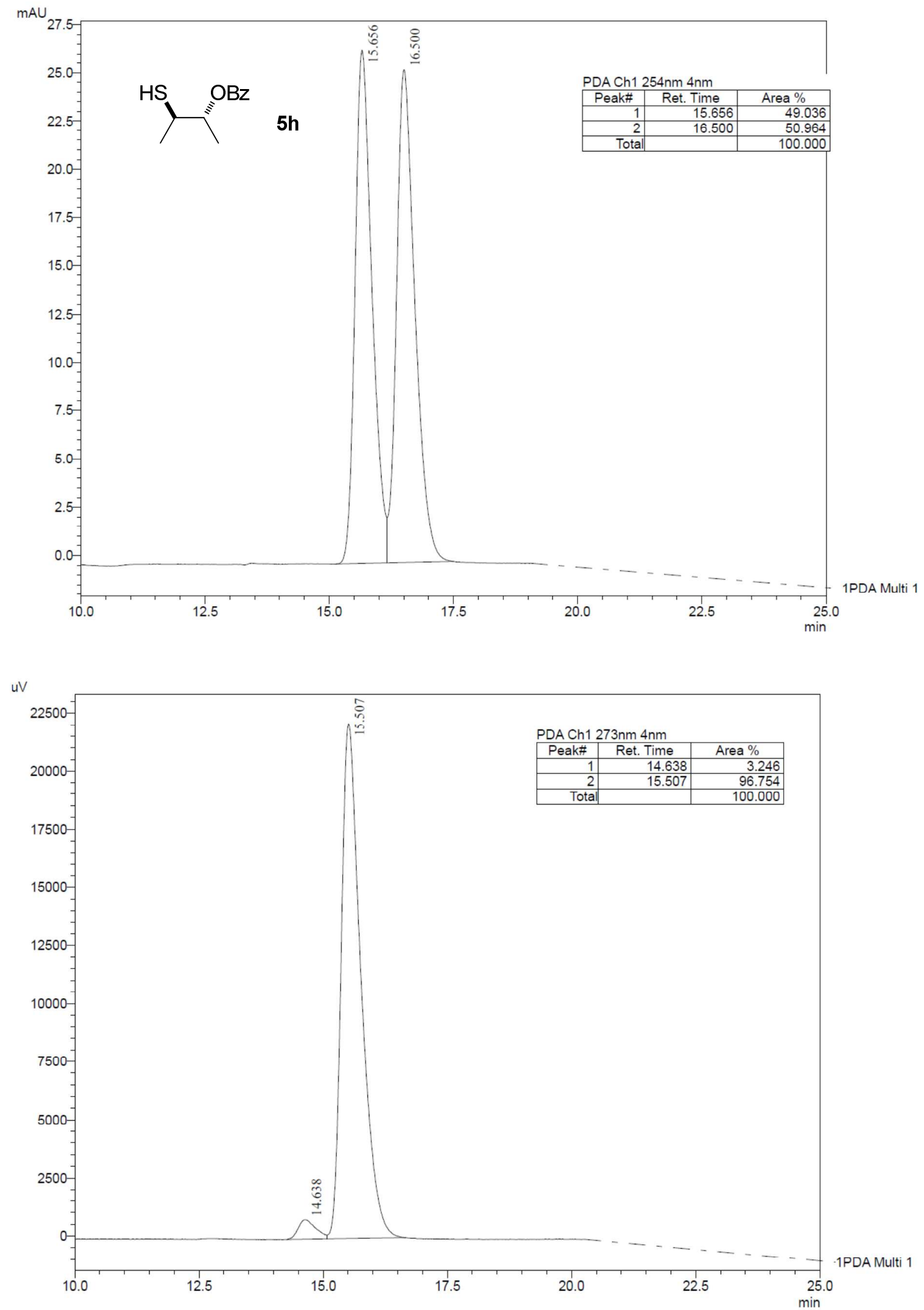

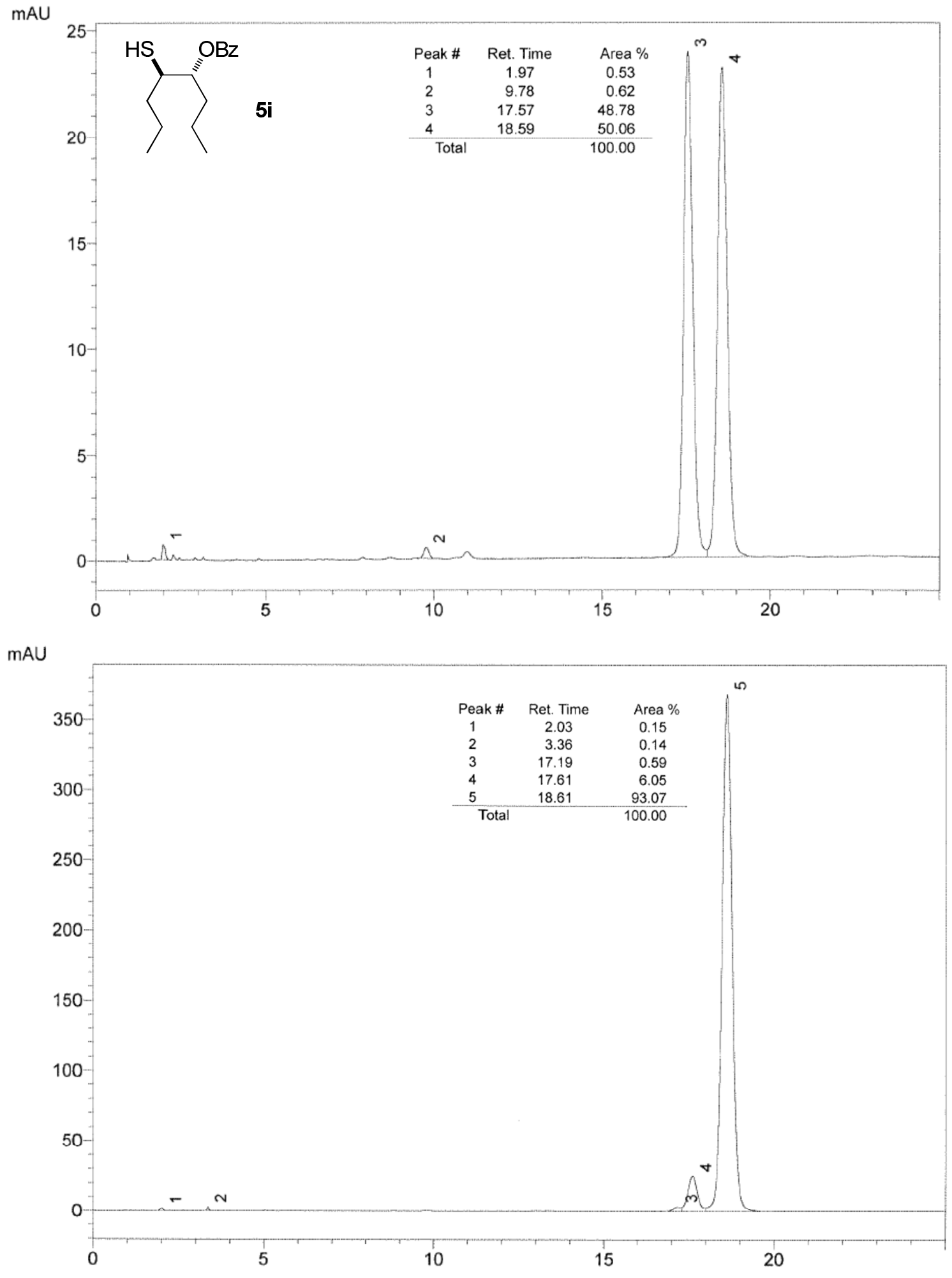

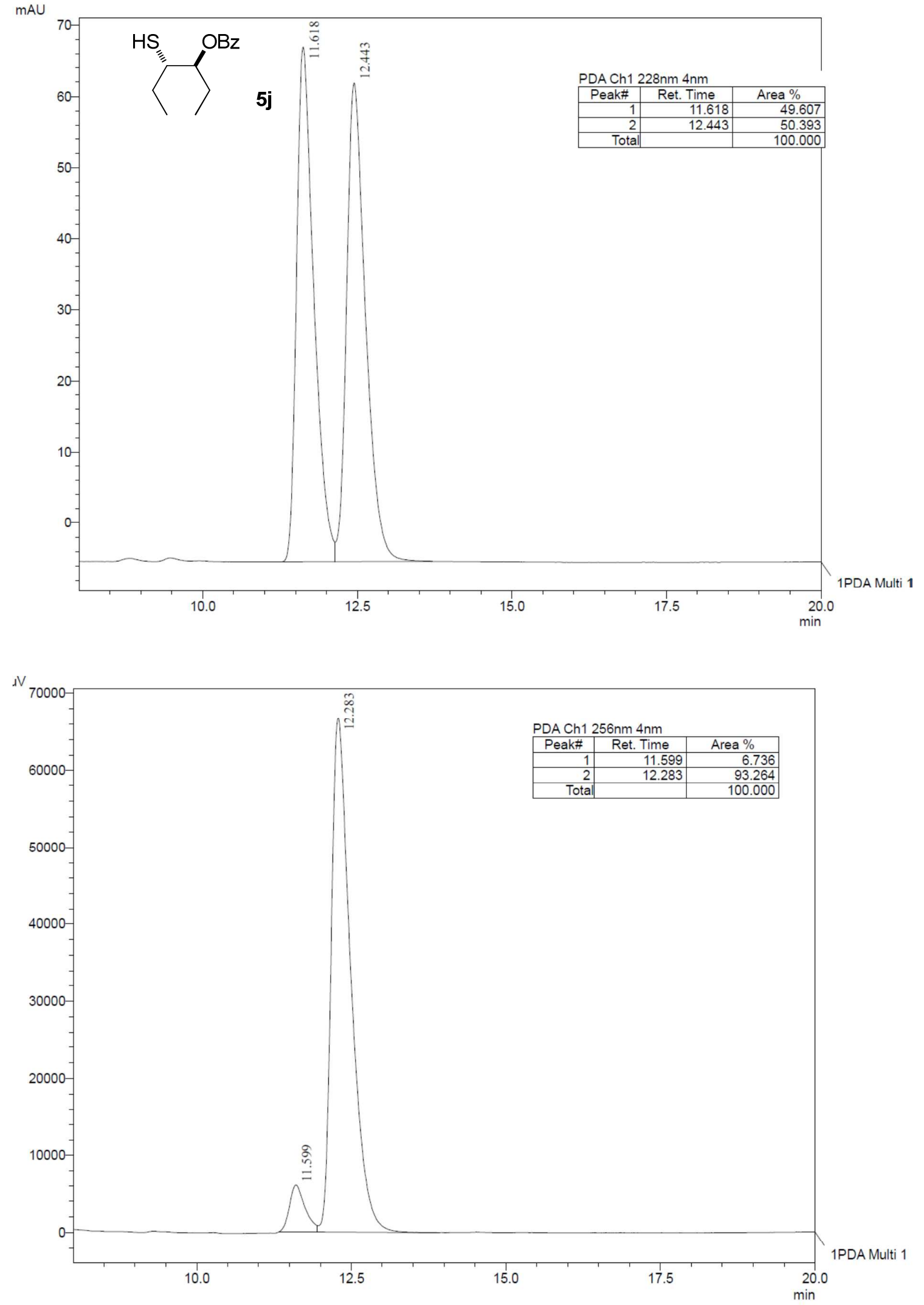

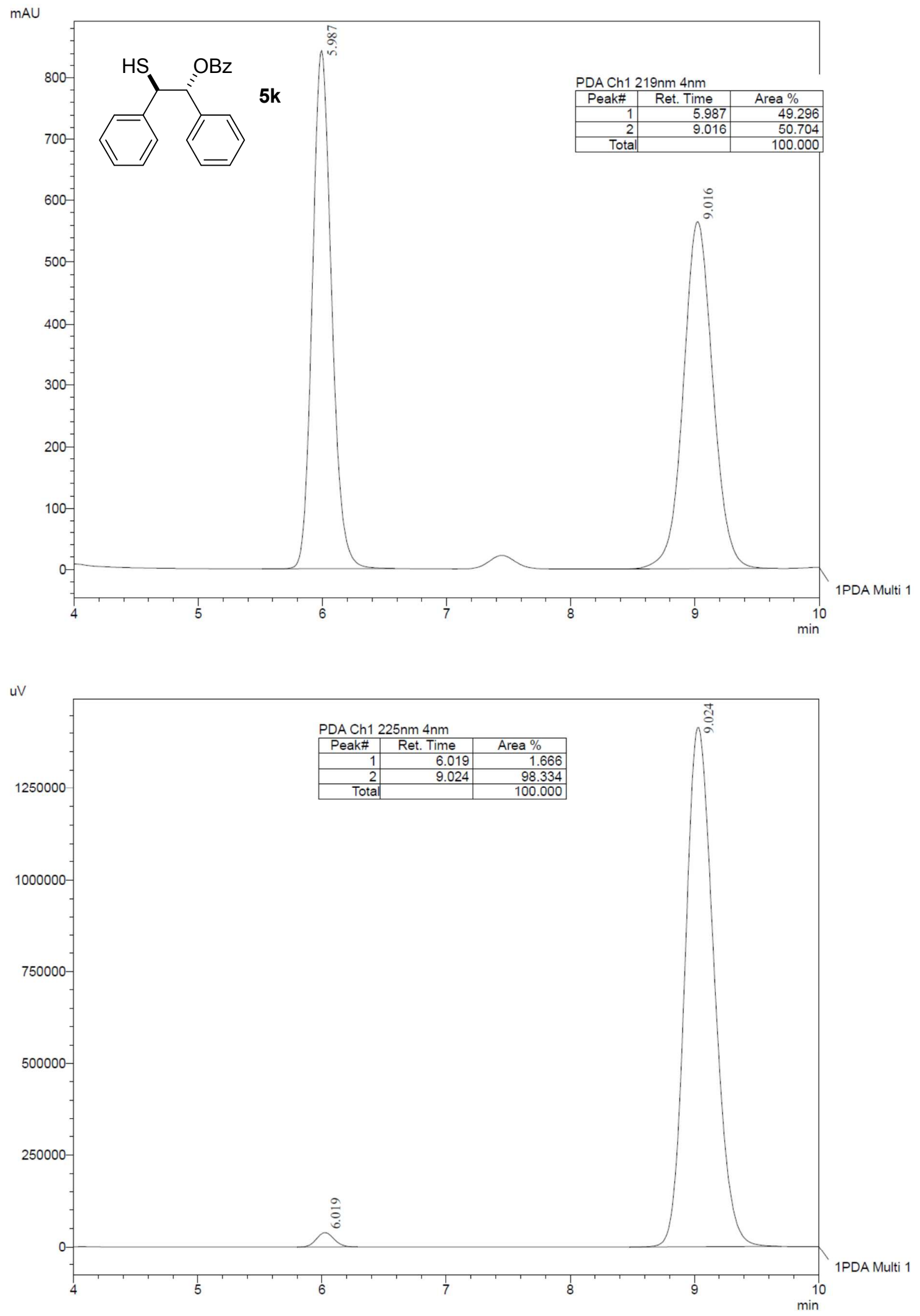

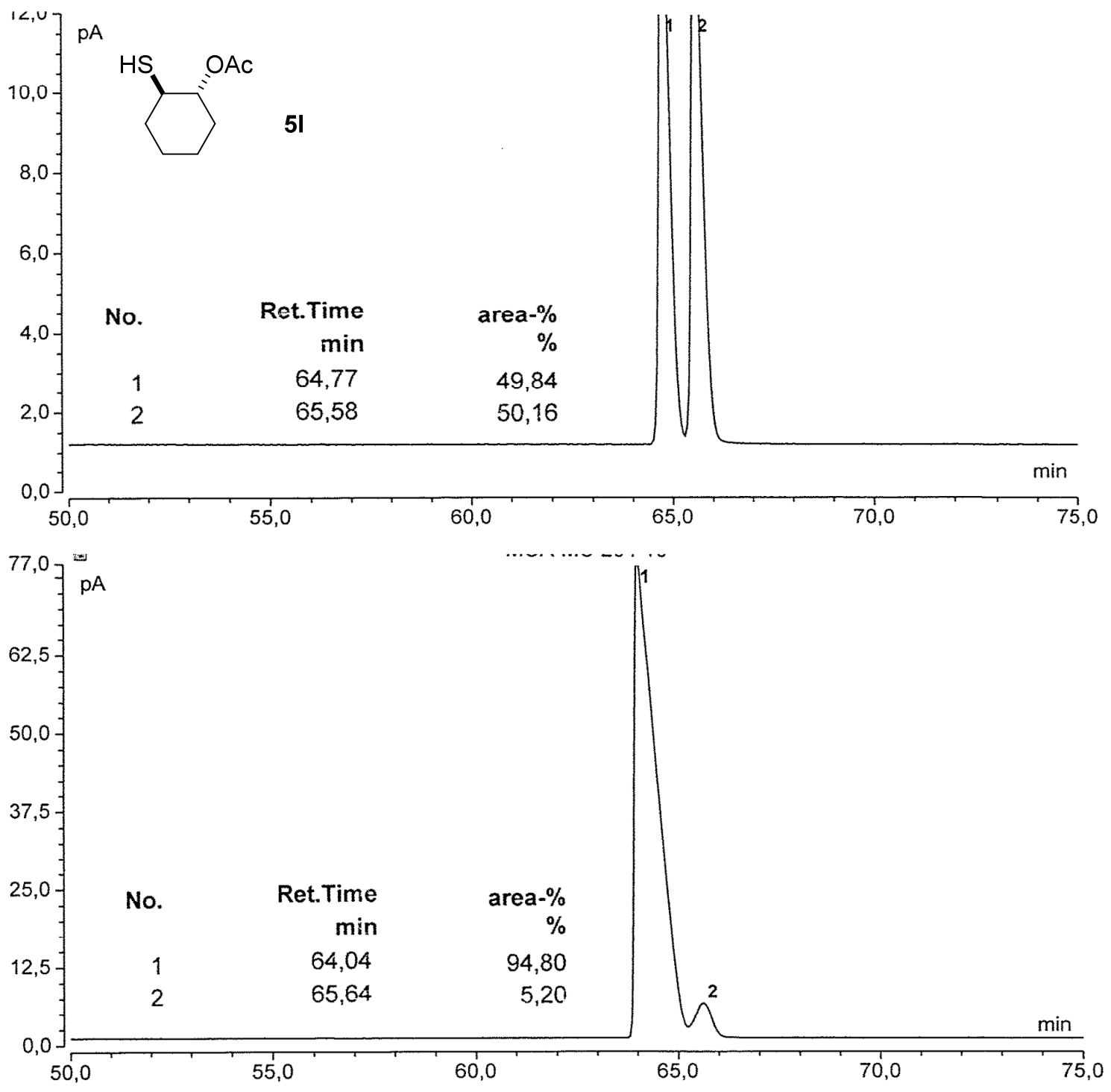

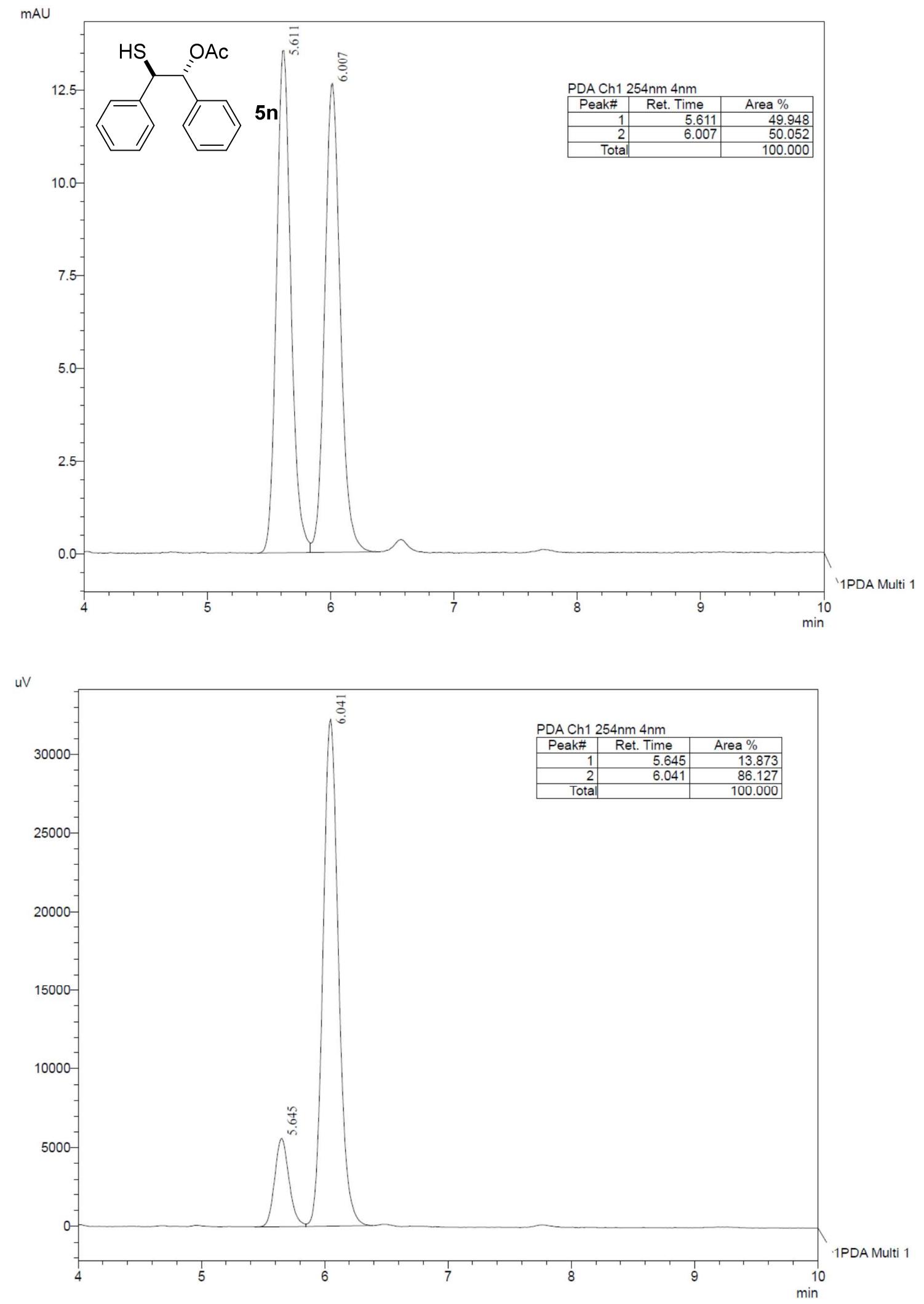

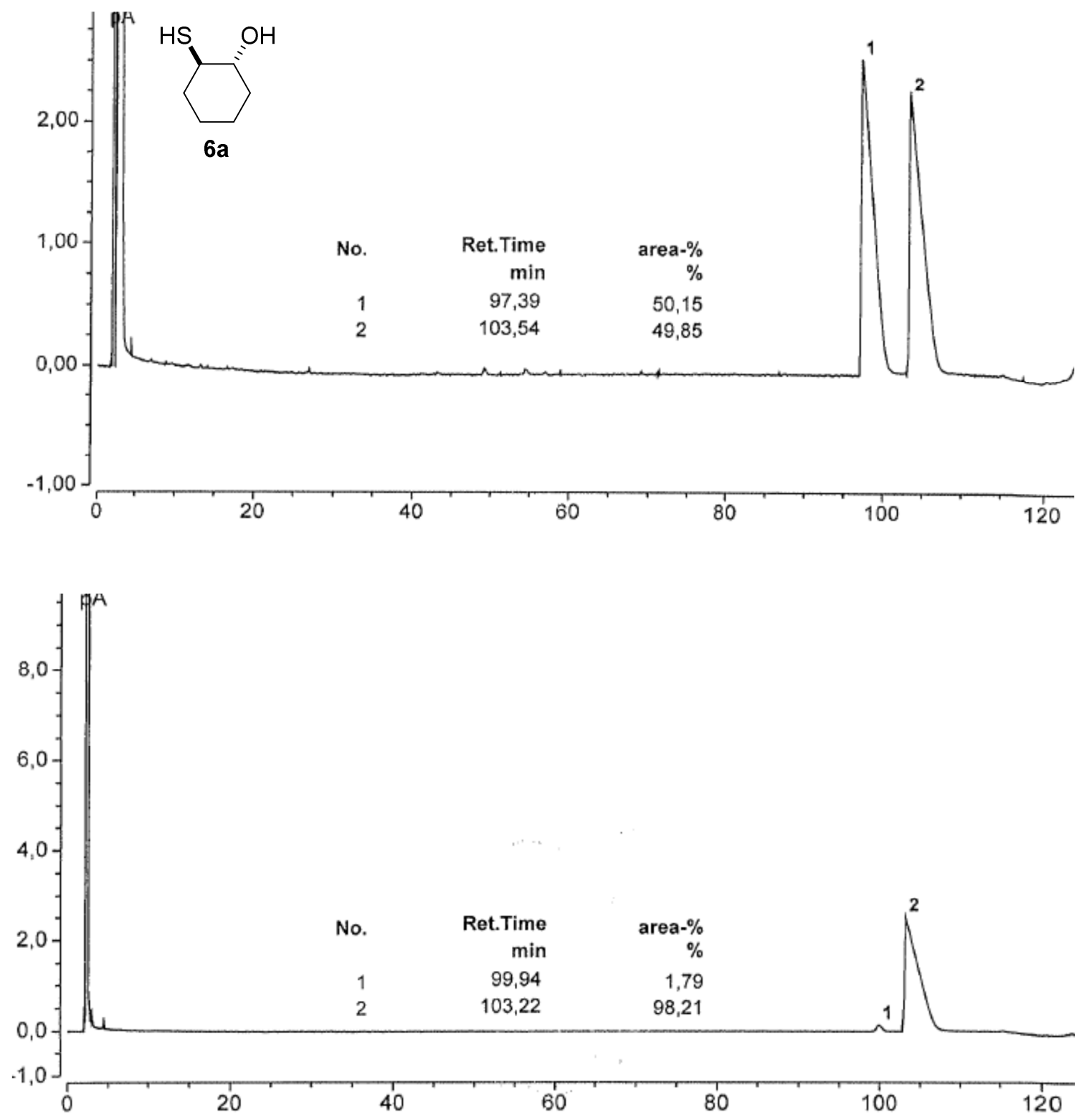

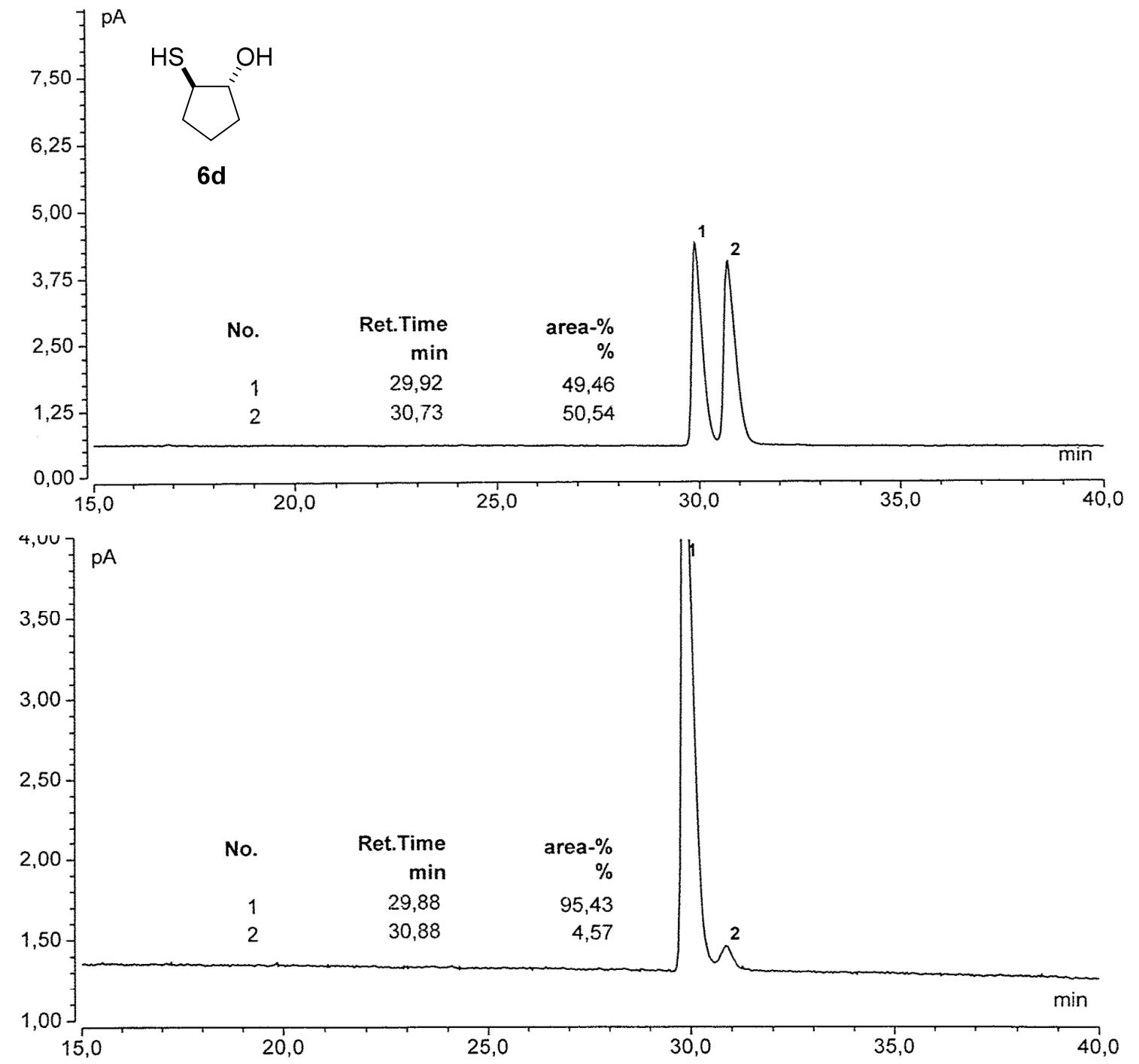